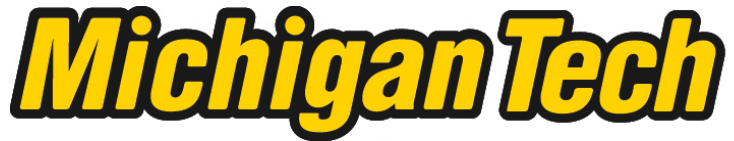 \\ Michigan Technological University Create the Future Digital Commons @ Michigan Tech
}

Dissertations, Master's Theses and Master's Reports - Open

Dissertations, Master's Theses and Master's

Reports

2013

Development of an In Situ Measurement Device for Obtaining Material Thermal Properties

Mark A. Hepokoski

Michigan Technological University

Follow this and additional works at: https://digitalcommons.mtu.edu/etds

Part of the Mechanical Engineering Commons

Copyright 2013 Mark A. Hepokoski

\section{Recommended Citation}

Hepokoski, Mark A., "Development of an In Situ Measurement Device for Obtaining Material Thermal Properties", Dissertation, Michigan Technological University, 2013.

https://doi.org/10.37099/mtu.dc.etds/745

Follow this and additional works at: https://digitalcommons.mtu.edu/etds

Part of the Mechanical Engineering Commons 


\title{
DEVELOPMENT OF AN IN SITU MEASUREMENT DEVICE FOR OBTAINING MATERIAL THERMAL PROPERTIES
}

\author{
By
}

Mark A. Hepokoski

\begin{abstract}
A DISSERTATION
Submitted in partial fulfillment of the requirements for the degree of DOCTOR OF PHILOSOPHY

In Mechanical Engineering-Engineering Mechanics
\end{abstract}

MICHIGAN TECHNOLOGICAL UNIVERSITY

2013 
This dissertation has been approved in partial fulfillment of the requirements for the Degree of DOCTOR OF PHILOSOPHY in Mechanical Engineering-Engineering Mechanics

Department of Mechanical Engineering-Engineering Mechanics

Dissertation Advisor: Jason R. Blough

Committee Member: $\quad$ Allen R. Curran

Committee Member: Jeffrey S. Allen

Committee Member: Stephen A. Hackney

Department Chair: $\quad$ William W. Predebon 
TABLE OF CONTENTS

Section

Page

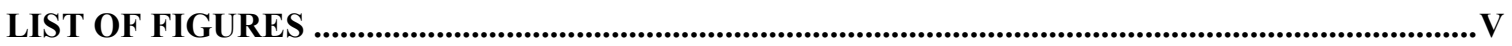

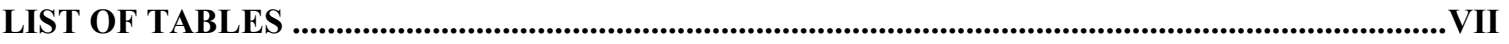

LIST OF EQUATIONS ...................................................................................................................... VIII

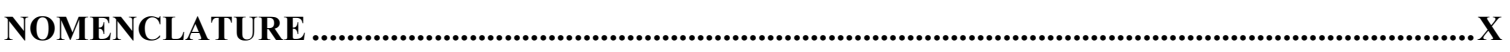

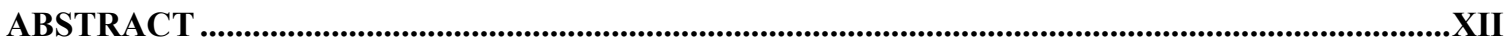

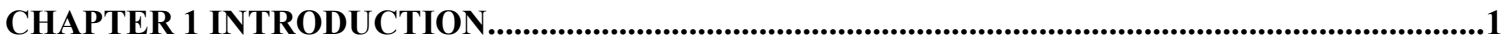

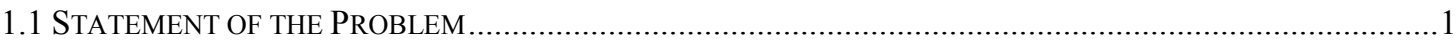

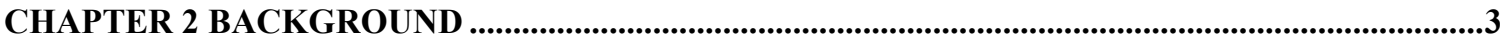

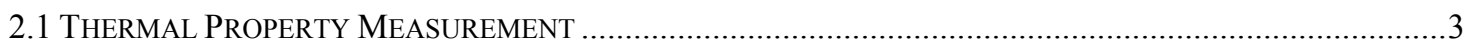

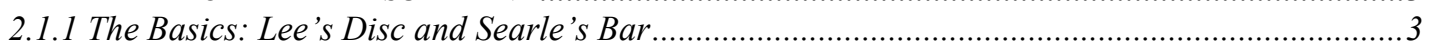

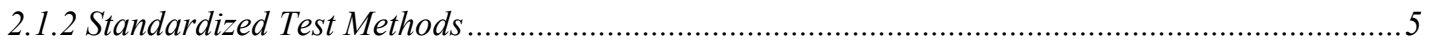

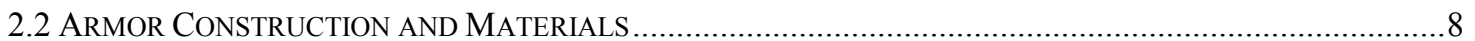

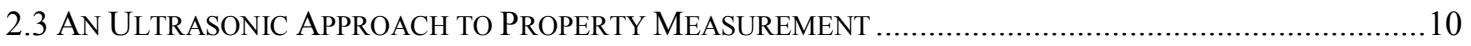

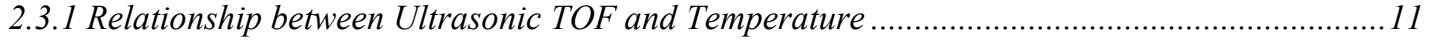

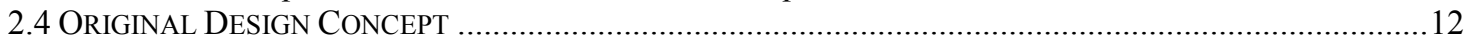

CHAPTER 3 OBJECTIVE ....................................................................................................................14

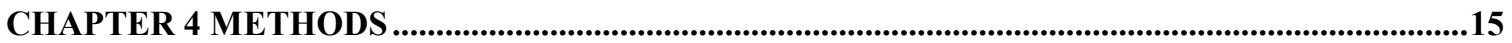

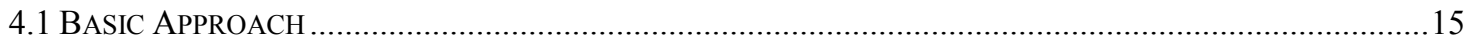

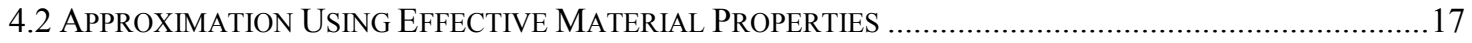

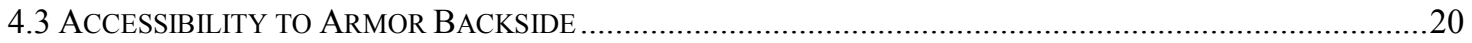

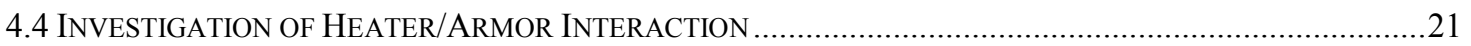

4.4.1 Development of an Ultrasound-based Property Measurement Device ........................................22

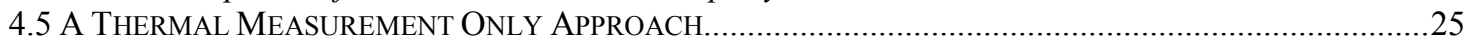

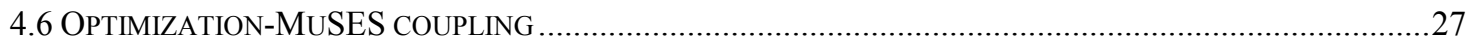

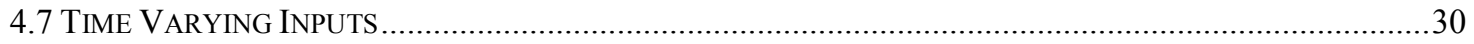

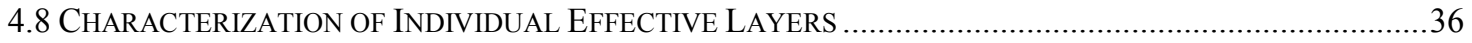

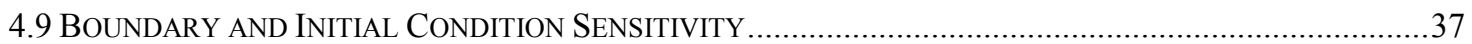

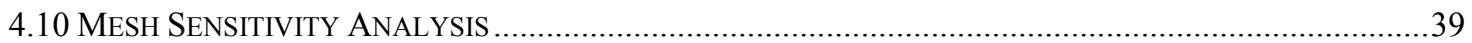

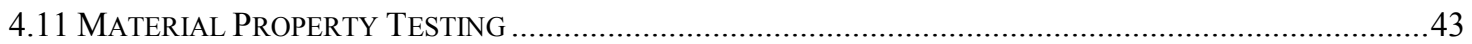

CHAPTER 5 DEVELOPMENT OF A PROPERTY MEASUREMENT DEVICE ...............................47

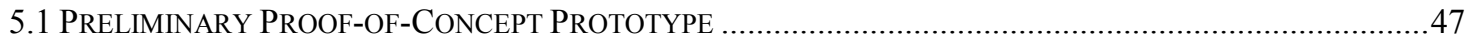

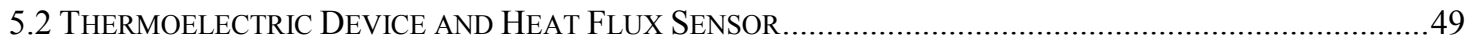

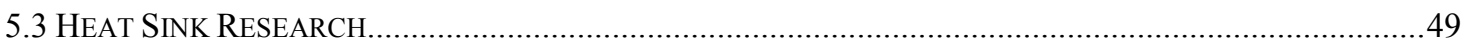

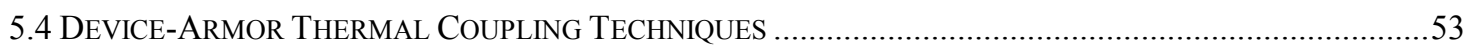

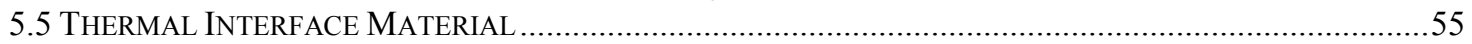

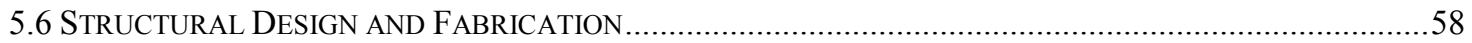

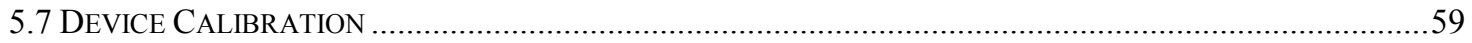

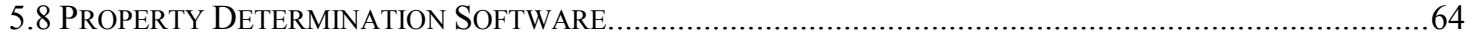

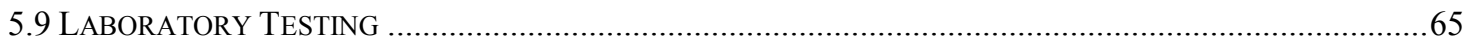


5.10 PRELIMINARY RESULTS AND ANALYSIS .

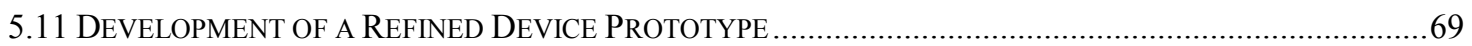

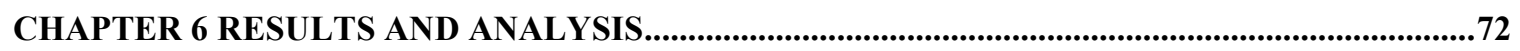

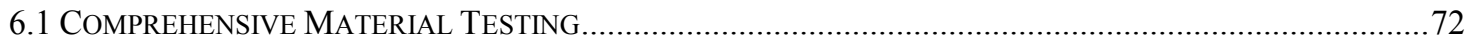

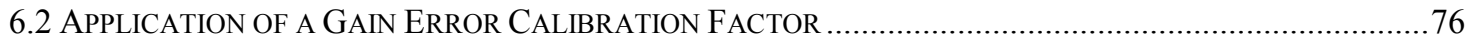

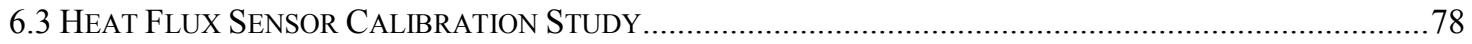

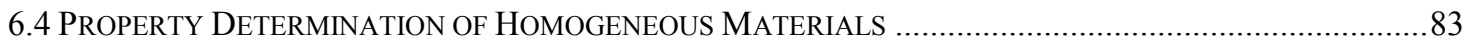

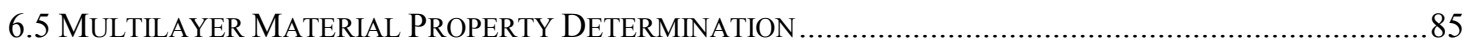

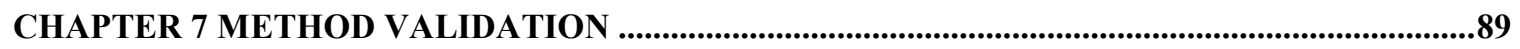

CHAPTER 8 CONCLUSION AND RECOMMENDATIONS FOR FUTURE WORK........................95

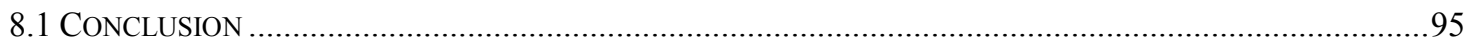

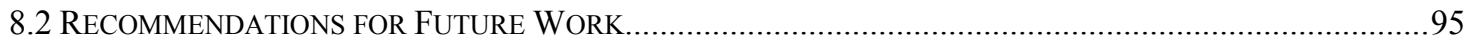

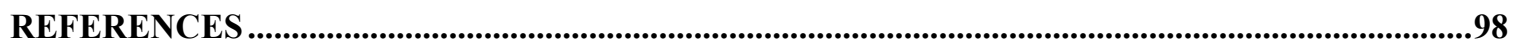

APPENDIX A SINUSOIDAL INPUTS AND RESPONSE: STARTING PHASE

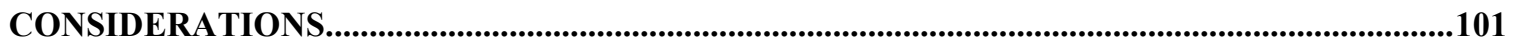

APPENDIX B DERIVATION OF A CLOSED FORM ULTRASONIC-BASED INTERNAL TEMPERATURE DISTRIBUTION EQUATION ............................................................................110

APPENDIX C HEAT SPREADER INVESTIGATION ..............................................................112 


\section{List of Figures}

Figure 1.1 - Example of a military ground vehicle thermal model ..............................................................1

Figure 2.1 - Illustration of the original design concept test setup .........................................................12

Figure 4.1 - Approximating armor thermal response using an effective resistance .....................................17

Figure 4.2 - Use of a single effective material to characterize a layered specimen ....................................19

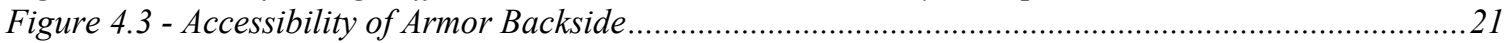

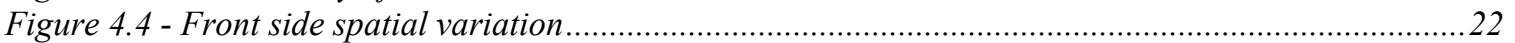

Figure 4.5 - Estimation of conductivity using the semi-infinite solid approximation....................................26

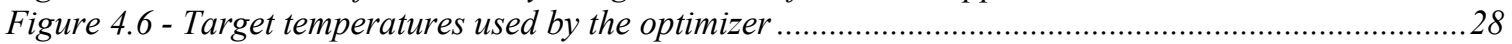

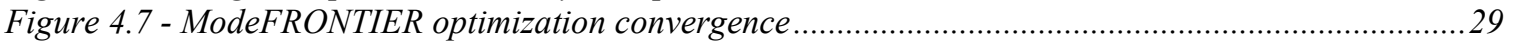

Figure 4.8 - Assessing the time until the backside temperature is affected ................................................ 30

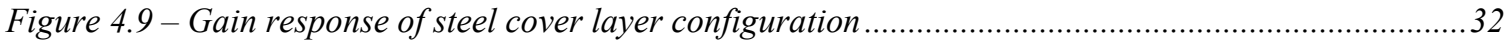

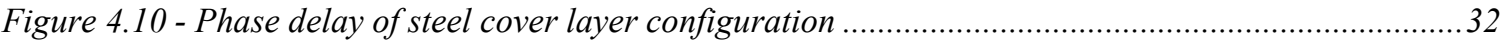

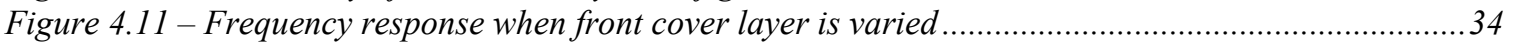

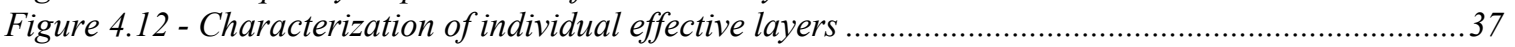

Figure 4.13-Coarse "quadrant" mesh with close-up of heater geometry .................................................40

Figure 4.14- The half quadrant mesh takes advantage of symmetry to reduce mesh size ............................ 41

Figure 4.15 - Mesh refinement needed to capture transients in highly conductive specimens......................41

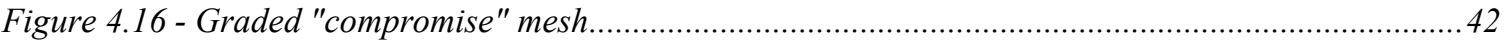

Figure 4.17- Example of a thermoelectric (Peltier) module .................................................................... 43

Figure 4.18- Sinusoidal operation of a thermoelectric device using temperature control............................44

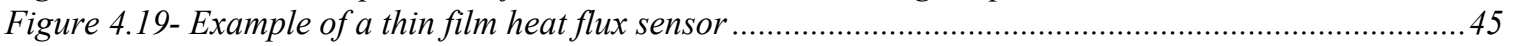

Figure 5.1 - Preliminary Measurement Device Prototype …….............................................................47

Figure 5.2 - TI MSP-EXP430F5438 test board with custom-built signal conditioner and H-bridge needed

to drive sinusoidal operation of thermoelectric device ..........................................................................48

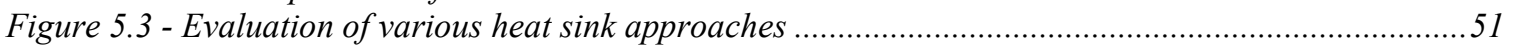

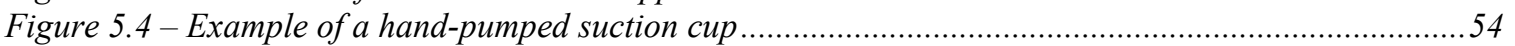

Figure 5.5 - Examples of various thermal interface materials ………...................................................5

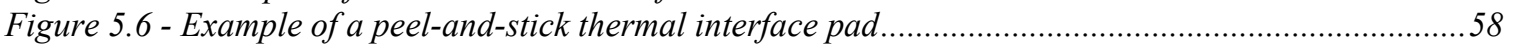

Figure 5.7 - Structural component fabricated by Motolla, LLC ............................................................59

Figure 5.8 - Temperature measurement diagram showing cold junction compensation ............................60 60

Figure 5.9-Direct correlation between ADC counts and T-Type thermocouple voltage.............................61

Figure 5.10-Correction for gain and offset error of the embedded T-Type thermocouple..........................62

Figure 5.11- Thermistor temperature correlation to voltage-based ADC counts ......................................63

Figure 5.12- ADC Counts to voltage correlation for the heat flux sensor ...............................................64

Figure 5.13- Property determination code graphical user interface ……….........................................65

Figure 5.14 - Testing of a homogenous aluminum sheet (1'x1.5'x1/2") ..................................................66

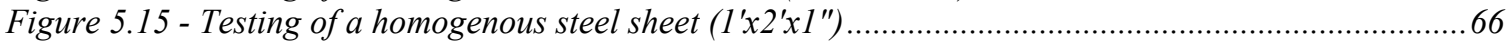

Figure 5.16 - Testing of a homogenous HDPE sheet (1'x2'x3/4")........................................................66

Figure 5.17- Correlation between measured temperaure data and model results......................................69

Figure 5.18 - Refined device prototype developed by Motolla, LLC...................................................... 70

Figure 5.19 - PAF control strategy reduces electrical noise and produces cleaner measurements ............. 71

Figure 6.1 - Gain response for range of applied sinusoidal heating frequencies ......................................... 73

Figure 6.2 - Phase response for range of applied sinusoidal heating frequencies ..................................... 74

Figure 6.3 - Simulated gain response compared with measurements .................................................... 75

Figure 6.4 - Simulated phase response compared with measurements ...................................................... 75

Figure 6.5 - Gain of the frequency response of simulated samples using a gain factor of 2.0 .................... 76

Figure 6.6 - Phase of the frequency response of simulated samples using a gain factor of 2.0 ...................77 
Figure 6.7 - HDPE calibration sample connected to heat flux sensor and Peltier module .........................78

Figure 6.8 - Heat flux sensor calibration setup .................................................................................... 78

Figure 6.9-Comparison of measured heat flux with simulated heat flux (constant power) ......................80

Figure 6.10 - Comparison of measured heat flux with simulated heat flux (sinusoidal power)..................81

Figure 6.11 - Thermal image showing non-uniform temperature at Peltier module surface......................82

Figure 6.12 - Ratio of calculated to measured heat flux ............................................................................8

Figure 6.13 - Testing of a steel over HDPE layered stack-up ...............................................................85

Figure 6.14 - Multilayer material thermal property determination......................................................8 87

Figure 7.1 - Patria armored vehicle finite-element mesh ..............................................................8

Figure 7.2 - Thermal (above) and radiance (below) predictions of a Patria military vehicle using measured

thermal properties of a steel/HDPE stackup as armor ...................................................................91

Figure 7.3 - Comparison of predicted radiances using different armor materials...................................99

Figure 7.4 - DTRSS contrast results comparison among permutations of the model with various armor

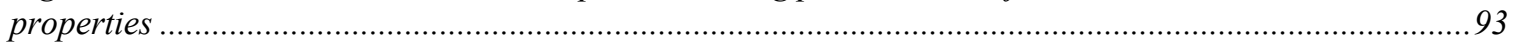

Figure A.1 - Positive sine input produces response that is shifted up along ordinate axis .......................102

Figure A.2 - Negative sine input produces response that is shifted down along ordinate axis ..................102

Figure A.3 - Cosine input produces response that oscillates about its starting temperature....................103

Figure A.4 - Response for small capacitance or lumped capacitance system given a sine input...............104

Figure A.5 - Response for small capacitance or lumped capacitance system given a cosine input............105

Figure A.6 - Accumulated heat input for different sinusoidal functions................................................ 106

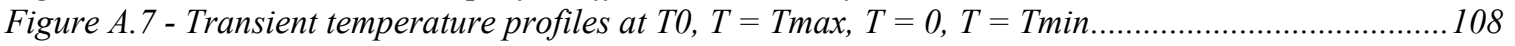

Figure C.1 - Copper heat spreader placement.................................................................................. 113

Figure C.2 - Constant power supplied to Peltier module with integrated copper heat spreaders .............113

Figure C.3 - Sinusoidal operation of Peltier module with integrated copper heat spreaders....................114 
List of Tables

\section{List of Tables}

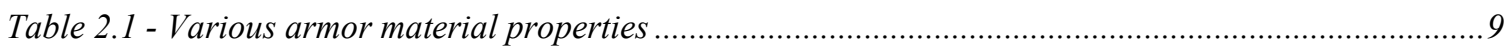

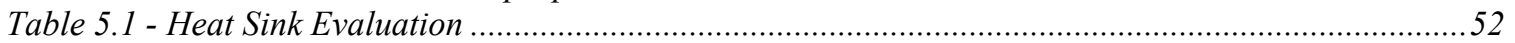

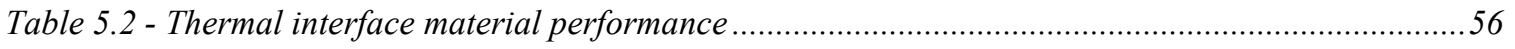

Table 5.3 - Results of preliminary homogenous material testing ...............................................................67

Table 6.1 - Materials and layered stack-ups used for testing ……........................................................ 72

Table 6.2- Material property results obtained from 3/4" HDPE at different frequencies ............................8 84

Table 6.3- Results of multilayer material property determination of a steel over HDPE stack-up..............87 


\section{List of Equations}

$$
\begin{aligned}
& G=2 L / c_{0}-\frac{(\alpha+\gamma)}{c_{0}} \int_{0}^{L} \theta(x) d x \\
& \theta(x)=T(x)-T_{0} \quad \text { (2.2) } \\
& \rho c_{p} \frac{\partial T}{\partial t}=k \nabla^{2} T \\
& -k \nabla T=q_{\text {env }}^{\prime \prime} \quad \text { (4.2) } \\
& \Delta x \rho_{1} c_{p, 1} \frac{\left(T_{1}-T_{1}^{\prime}\right)}{\Delta t}=q_{\text {front }}^{\prime \prime}+\frac{k_{1}}{2 \Delta x}\left(T_{2}-T_{1}\right) \\
& \Delta x\left(\rho_{j} c_{p, j}+\rho_{j+1} c_{p, j+1}\right) \frac{\left(T_{i+1}-T_{i+1}^{\prime}\right)}{\Delta t}=\frac{k_{j}}{2 \Delta x}\left(T_{i}-T_{i+1}\right)+\frac{k_{j+1}}{2 \Delta x}\left(T_{i+2}-T_{i+1}\right) \\
& \Delta x \rho_{m} c_{p, m} \frac{\left(T_{n}-T_{n}^{\prime}\right)}{\Delta t}=\frac{k_{m}}{2 \Delta x}\left(T_{n-1}-T_{n}\right)+q_{b a c k}^{\prime \prime} \\
& R_{\text {eff }}=\sum_{i}^{n} L_{i} / k_{i} \\
& C_{e f f}=\sum_{i}^{n}\left(\rho V c_{p}\right)_{i} \\
& k \rho c_{p}=\frac{4 t}{\pi}\left(\frac{q_{s}^{\prime \prime}}{T_{s}-T_{i}}\right)^{2} \\
& f^{\prime}\left(x_{i}\right)=\frac{f\left(x_{i-2}\right)-8 f\left(x_{i-1}\right)+8 f\left(x_{i+1}\right)-f\left(x_{i+2}\right)}{12 h} \\
& \Delta T_{R S S}=\sqrt{\left(\overline{T_{T}}-\overline{T_{B}}\right)^{2}+\sigma_{T}^{2}} \\
& P_{i n}=\frac{d E_{s y s}}{d t} \quad(\text { A.1) } \\
& E_{s y s}=-A \cos (\omega t)+A \quad \text { (A.2) } \\
& E_{s y s}=A \sin (\omega t) \quad \text { (A.3) } \\
& \Delta x \rho c_{p, 1} \frac{\left(T_{1}-T_{1}^{\prime}\right)}{\Delta t}=q^{\prime \prime}+\frac{k_{1}}{2 \Delta x}\left(T_{2}-T_{1}\right) \quad \text { (A.4) } \\
& \Delta x\left(\rho_{1} c_{p, 1}+\rho_{2} c_{p, 2}\right) \frac{\left(T_{2}-T_{2}^{\prime}\right)}{\Delta t}=\frac{k_{1}}{2 \Delta x}\left(T_{1}-T_{2}\right)+\frac{k_{2}}{2 \Delta x}\left(T_{3}-T_{2}\right) \\
& G=\frac{2 L}{c_{i}}-\frac{(\alpha+\gamma)}{c_{i}} \int_{0}^{L} T(x)-T_{i} d x \quad \text { (B.1) }
\end{aligned}
$$




$$
\begin{aligned}
& T(x)=\frac{q^{\prime \prime}}{k} x+T_{s} \\
& q^{\prime \prime}=\frac{2 k}{L}\left[\frac{2 L-c_{i} G}{\alpha+\gamma}-T_{s} L-T_{i} L\right] \\
& T(x)=\frac{2}{L^{2}}\left[\frac{2 L-c_{i} G}{\alpha+\gamma}-T_{s} L-T_{i} L\right] x+T_{s} \\
& c_{i}=\frac{2 L}{G_{i}} \\
& T(x)=\frac{4}{L}\left[\frac{1-G / G_{i}}{\alpha+\gamma}-\frac{T_{s}-T_{i}}{2}\right] x+T_{s}
\end{aligned}
$$




\section{Nomenclature}

\begin{tabular}{|c|c|c|}
\hline Parameter & Description & Units \\
\hline$\alpha$ & Coefficient of linear expansion & {$[1 / \mathrm{K}]$} \\
\hline$c_{0}$ & Speed of sound at the reference state & {$[\mathrm{m} / \mathrm{s}]$} \\
\hline$C_{\text {eff }}$ & Effective thermal capacitance & {$[\mathrm{J} / \mathrm{K}]$} \\
\hline$c_{p}$ & Specific heat of a material & {$[\mathrm{J} / \mathrm{kg} \mathrm{K}]$} \\
\hline$E_{\text {sys }}$ & Energy of a system & {$[\mathrm{J}]$} \\
\hline$k$ & Thermal conductivity & {$[\mathrm{W} / \mathrm{m} \mathrm{K}]$} \\
\hline$L_{i}$ & Thickness of an individual material layer & {$[\mathrm{m}]$} \\
\hline$P_{\text {in }}$ & Input power & {$[\mathrm{W}]$} \\
\hline$\dot{\dot{q}}_{e n v}$ & Environmental heat transfer & {$[\mathrm{W}]$} \\
\hline$q_{s}^{\prime \prime}$ & Heat flux at a surface & {$\left[\mathrm{W} / \mathrm{m}^{2}\right]$} \\
\hline$R_{\text {eff }}$ & Effective thermal resistance & {$[\mathrm{K} / \mathrm{W}]$} \\
\hline$\rho$ & Density of a material & {$\left[\mathrm{kg} / \mathrm{m}^{3}\right]$} \\
\hline$t$ & Time & {$[\mathrm{s}]$} \\
\hline$T_{i}$ & Initial temperature & {$\left[{ }^{\circ} \mathrm{C}\right.$ or $\left.\mathrm{K}\right]$} \\
\hline$T_{s}$ & Surface temperature & {$\left[{ }^{\circ} \mathrm{C}\right.$ or $\left.\mathrm{K}\right]$} \\
\hline$\theta$ & Temperature difference from initial state & {$\left[{ }^{\circ} \mathrm{C}\right.$ or $\left.\mathrm{K}\right]$} \\
\hline$V$ & Volume & {$\left[\mathrm{m}^{3}\right]$} \\
\hline
\end{tabular}


Nomenclature 


\begin{abstract}
This thesis presents a methodology for measuring thermal properties in situ, with a special focus on obtaining properties of layered stack-ups commonly used in armored vehicle components. The technique involves attaching a thermal source to the surface of a component, measuring the heat flux transferred between the source and the component, and measuring the surface temperature response. The material properties of the component can subsequently be determined from measurement of the transient heat flux and temperature response at the surface alone. Experiments involving multilayered specimens show that the surface temperature response to a sinusoidal heat flux forcing function is also sinusoidal. A frequency domain analysis shows that sinusoidal thermal excitation produces a gain and phase shift behavior typical of linear systems. Additionally, this analysis shows that the material properties of sub-surface layers affect the frequency response function at the surface of a particular stack-up. The methodology involves coupling a thermal simulation tool with an optimization algorithm to determine the material properties from temperature and heat flux measurement data. Use of a sinusoidal forcing function not only provides a mechanism to perform the frequency domain analysis described above, but sinusoids also have the practical benefit of reducing the need for instrumentation of the backside of the component. Heat losses can be minimized by alternately injecting and extracting heat on the front surface, as long as sufficiently high frequencies are used.
\end{abstract}




\section{Chapter 1}

\section{Introduction}

\subsection{Statement of the Problem}

To create a realistic thermal model of an object of interest, such as a commercial or military ground vehicle, it is necessary to first obtain the thermal properties of all of the constituent components. In most cases, the materials of the individual components are known, and it is therefore sufficient to obtain the necessary thermal properties (conductivity and specific heat) from the published literature in order to begin model development.

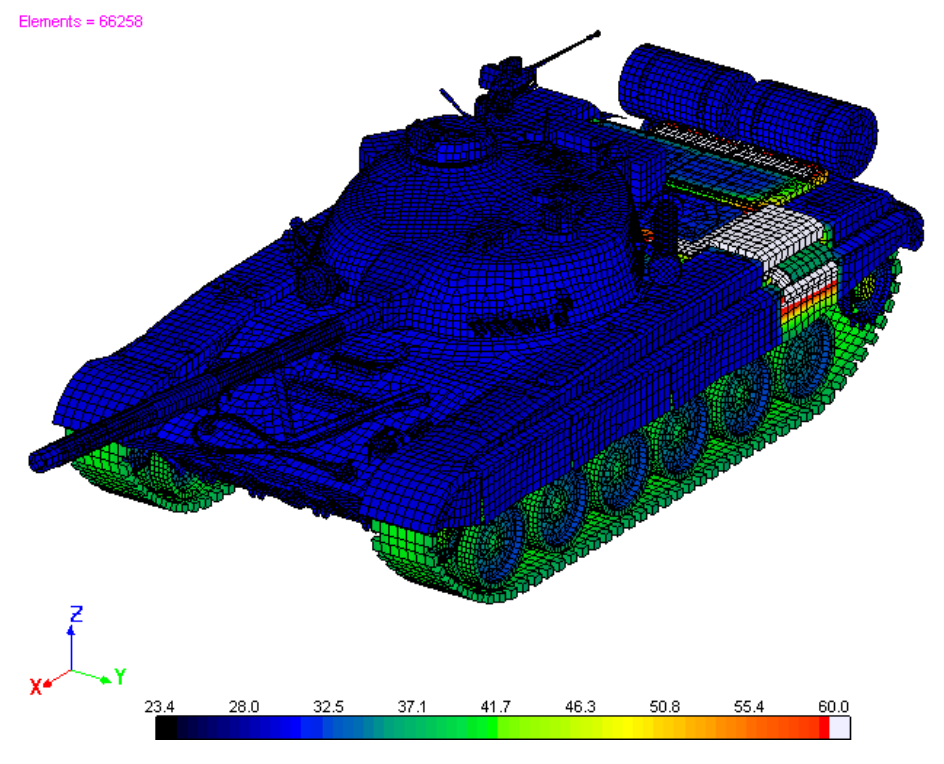

Figure 1.1 - Example of a military ground vehicle thermal model 
Military ground vehicles on the other hand are especially difficult to model because they are typically outfitted with one or more layers of armor that can significantly affect the heat transfer from the vehicle to the environment. To further complicate matters, the material make-up of these armor panels, including the thermal properties associated with them, are not readily obtainable from either the manufacturer or the military due to the inherent sensitive nature of their composition (from both a proprietary and intelligence/security standpoint).

Since armor stack-ups can vary from vehicle to vehicle, even among vehicles of the same type, field testing of a particular vehicle of interest is the ideal method of obtaining material thermal properties for modeling purposes. However, since these components cannot be removed from the vehicles of interest, traditional test methods cannot be employed to obtain the necessary thermal properties. 


\section{Chapter 2}

\section{Background}

\subsection{Thermal Property Measurement}

There are a wide variety of established methods for measuring the thermal properties of materials. Selection of a particular method depends on the material type, temperature range, and property of interest, i.e., thermal resistance (or conductivity) or thermal capacitance (specific heat). When only the thermal resistance is desired, steady-state techniques are sufficient; however, for thermal capacitance measurements, transient techniques must be employed.

\subsubsection{The Basics: Lee's Disc and Searle's Bar}

The two most common techniques for measuring thermal conductivity are the Searle's Bar Method and the Lee's Disc Method, the former being more applicable to thermally conductive specimens while the latter is more applicable to thermally resistive specimens.

The Searle's Bar Method incorporates a test procedure that imposes a constant temperature difference between two ends of a cylindrical test specimen so that the thermal conductivity can be obtained by solving the one dimensional steady-state heat 
conduction equation. The particular technique for maintaining the temperature difference between the two ends of the bar is not fundamental to the method and can therefore vary depending on the experimental approach, provided that the heating is performed in such a way as to allow the heat flow through the bar to be measured. In order to fulfill the adiabatic boundary condition assumption, both the device must be sufficiently insulated and the specimen must thermally conductive.

The Lee's Disc Method addresses the limitations inherent for testing thermally resistive samples with the Searle's Bar Method by making use of a very thin circular specimen that is sandwiched between a hot surface and a cold surface. The small surface area along the perimeter of the disc reduces the ratio of heat lost to the surroundings to the heat traveling through the bar, which limits measurement error in the conductivity calculation. However, in contrast to the Searle's Bar Method, the experiment for the Lee's Disc Method is divided up into two phases: The first phase is a steady-state experiment similar to the Searle's Bar Method; the second phase immediately follows in which the sample is either heated or cooled to $\sim 5^{\circ} \mathrm{C}$ above or below its previous steady-state value. The conductivity of the specimen is then obtained from the time rate of change of the disc temperature, mass, dimensions, and the hot and cold temperatures of the surfaces that were measured during the steady-state phase of the experiment. 


\subsubsection{Standardized Test Methods}

A number of international organizations (e.g. ISO, ASTM, IEEE, etc.) have compiled procedures to help standardize the measurement of material thermal properties.

For example, ASTM D5930 "Standard Test Method for Thermal Conductivity of Plastics by Means of a Transient Line-Source Technique" maps out step-by-step instructions on how to perform material testing according to the line-source technique [1] [3] [6]. This transient method for determining thermal conductivity involves a line heat source that is inserted at the center of the specimen. When electrical current is supplied to the source to generate heat, a thermal wave propagates radially into the specimen, facilitating characterization of the heat transfer within the specimen in terms of a one dimensional transient conduction equation. During the course of the experiment, the temperature rise measured at the source will eventually stabilize to form a linear relationship with time on a logarithmic scale. The conductivity of the specimen can then be obtained directly from knowledge of the applied heat rate and the temperature response slope using a simple correlation.

The same underlying principle used in the line source technique has been applied in the development of various techniques and associated devices for determining the thermal properties of materials commonly encountered in electrical and civil engineering applications. For example, Krishnaiah et al. have developed a methodology to determine 
Chapter 2 - Background

the thermal properties of rocks [10], which is based on the "Transient heat method" [1]. By inserting a thin thermal probe, which is outfitted with a thermocouple, into a rock sample and supplying it with a known electrical power, the temperature response to the subsequent heat input can be used to back out the thermal resistance and diffusivity of the rock sample. There are several related standard procedures for measuring soil thermal properties using similar methods, e.g. IEEE Standard 442-1981 “Guide for Soil Thermal Resistivity measurements" [4] and ASTM D5334 "Standard Test Method for Determination of Thermal conductivity of Soil and Soft Rock by Thermal Needle Probe Procedure" [5].

A method known as the Divided Bar of Comparative Cut Method is described in ASTM E1225, "Standard Test Method for Thermal Conductivity of Solids by Means of the Guarded-Comparative-Longitudinal Heat Flow Technique" [1]. This method involves placing a bar of sample material in series with a bar with known material properties. Heat is applied to one end of the divided bar and the thermal conductivity of the sample is evaluated by measuring the steady-state temperature gradient across the two bars. The amount of heat applied to the sample does not need to be known to obtain the conductivity.

The Heat Flow Meter Method extends the divided bar method by making use of a heat flux sensor to measure the heat flow through the sample. This method is described in ASTM E1530 "Standard Test Method for Evaluating the Resistance to Thermal 
Chapter 2 - Background Transmission of Materials by the Guarded Heat Flow Meter Technique" [11] and ASTM C518 "Standard Test method for Steady-State Thermal Transmission Properties by Means of a Heat Flow Meter Apparatus" [12].

The Guarded Hot Plate Method is widely used for the measurement of thermally insulative materials. This method can be applied to large specimens in the form of flat slabs. This simple device measures the steady state heat transfer and temperature response of a material by means of an electrically heated metering section surrounded laterally by a guard heater section controlled using differential thermocouples. Detailed descriptions of this technique and its associated device are available in ISO 8302 "Thermal insulation - Determination of steady-state thermal resistance and related properties - Guarded hot plate apparatus" [13] and ASTM C177 "Standard Test Method for Steady-State Heat Flux Measurements and Thermal Transmission Properties by Means of the Guarded-Hot-Plate Apparatus" [14].

ISO 22007 describes three techniques to determine the thermal conductivity and diffusivity of plastics: i) Transient plane heat source (hot disc) method; ii) Temperature wave analysis method; and iii) Laser flash method [7] [8] [9]. The principle behind the first of these methods, the hot disc method, involves applying a stepwise heat pulse via an electrical current through a hot-disc probe embedded within the test specimen. The transient temperature response is correlated with the material properties from knowledge of the probe construction and assumed boundary conditions. The Temperature Wave 
Analysis (TWA), is a recently developed method that relies on the frequency dependency of thermal diffusivity as a function of temperature. The laser flash method provides a technique to obtain thermal diffusivity by measuring the back side temperature response of a thin specimen that is exposed to a short energy pulse.

\subsection{Armor Construction and Materials}

A literature review was conducted to determine the extent of information that is available in the public domain regarding armor recipes and material properties. Unfortunately (although not unexpected), very little information on actual armor recipes was found available in the published literature. However, based on the information gathered and further confirmed by individuals that work in the armor industry, the Army Composite Armor Vehicle (CAV) recipe can be assumed to be representative of many armor recipes:

\section{Army Composite Armor Vehicle (CAV) Construction}

- Thin protective PMC (polymer matrix composite) outer face-sheet

- Thick ceramic/alumina armor tiles

- $\quad$ Thin EPDM (ethylene propylene diene monomer) rubber

- Thick structural composite

- Thin-to-moderate fire-protective "spall” phenolic inner liner 


\section{Northrop Grumman Patent 6135006}

Armor recipes can even be found within patent disclosures such as the following fiberreinforced ceramic matrix composite (FRCMC) armor developed by Northrop Grumman. This armor recipe is constructed by laying pairs of hard ceramic layers and less-thick ductile layers. The thin ductile layers consist of woven 3M Nextel 312 alumina-boriasilica fiber sheets and the hard ceramic layers consist of black glass silicon-carboxyl resin.

Very little information on the properties for armor materials can be obtained from the public domain, as was the case for armor recipes. Additionally, the material properties for an individual material may have a wide variation of thermal properties. For example, the thermal conductivity of silicon-carbide can vary by over an order of magnitude, indicating that knowledge of a particular armor recipe may not be sufficient to accurately characterize the thermal response of the armor as constructed.

\section{Table 2.1 - Various armor material properties}

\begin{tabular}{|l|l|l|l|l|l|l|}
\hline Material & $\mathrm{k}[\mathrm{W} / \mathrm{m} / \mathrm{K}]$ & $\mathrm{Cp}[\mathrm{J} / \mathrm{kg} / \mathrm{K}]$ & $\mathrm{E} \quad[\mathrm{GPa}]$ & Density $[\mathrm{kg} / \mathrm{m} 3]$ & $\mathrm{c}[\mathrm{km} / \mathrm{s}]$ & $\mathrm{CTE} \quad[\mathrm{K}]$ \\
\hline Boron carbide & $27-29$ & 1854 & $440-483$ & 2510 & $13.2-14.5$ & $2.6 \mathrm{E} 6-5.6 \mathrm{E} 6$ \\
\hline Silicon carbide & $10-125$ & 670 & $410-476$ & $3100-3190$ & $11.5-12.2$ & $3.0 \mathrm{E} 6-4.78 \mathrm{E} 6$ \\
\hline Timetal 6.4 titanium & 7.2 & 560 & $106-114$ & 4420 & $4.9-5.1$ & $8.8 \mathrm{E} 6-9.2 \mathrm{E} 6$ \\
\hline Kevlar 49 & 0.2 thru, 1-2 axial & 1000 & $24-77$ & 1440 & $4.1-7.3$ & $6.0 \mathrm{E} 7-1.1 \mathrm{E} 8$ \\
\hline
\end{tabular}




\subsection{An Ultrasonic Approach to Property Measurement}

Research was also performed to determine the extent of material properties obtainable from using ultrasonic wave propagation through an armor sample. The basis of the ultrasound approach is that the time between when an ultrasonic wave is emitted and its echo received is determined by the properties of a particular material, specifically, the speed of sound of that material at some reference state. In addition, multilayered objects produce multiple echoes, indicating that the assessment of material properties within a layered sample may be feasible using ultrasound.

A typical application of ultrasonic devices is to obtain the number of layers within a layered material as well as the individual thicknesses of these layers. Since the material associated with each layer is usually known beforehand, an ultrasonic technician simply provides the device with the known material makeup of the specimen, which embedded software uses to access built-in material properties databases so that the TOF (time-offlight), or $G$ (s), between the emission of the wave and the receipt of the echo can be expressed in terms thickness (m). These devices tend to be reasonably priced; for example, a higher end ultrasonic thickness gage from Olympus only costs somewhere between $\$ 4 \mathrm{~K}$ to $\$ 5 \mathrm{~K}$. 


\subsubsection{Relationship between Ultrasonic TOF and Temperature}

Besides the estimation of thickness and layer counts, techniques for estimating the internal temperature distribution using ultrasound can also be found in the published literature [16]. Ultrasonic temperature measurement is typically employed in nondestructive-evaluation (NDE) applications, such as those involving testing on living tissue.

The relationship between ultrasonic time-of-flight $(G)$ and the temperature change from an initial state $(\theta)$ can be expressed as follows,

$$
G=2 L / c_{0}-\frac{(\alpha+\gamma)}{c_{0}} \int_{0}^{L} \theta(x) d x
$$

where $L$ is the thickness of the layer (m), and $\alpha, \gamma$, and $\mathrm{c}_{0}$ are thermally dependent material properties: $\alpha$ is the coefficient of linear expansion $\left(\mathrm{K}^{-1}\right), \gamma$ is the Young's Modulus change with temperature $\left(\mathrm{K}^{-1}\right)$, and $\mathrm{c}_{0}$ is the speed of sound at the reference state $(\mathrm{m} / \mathrm{s})$ [17]. The temperature difference, $\theta,(\mathrm{K})$ is defined as the difference in temperature at any point at a distance, $\mathrm{x}$, through the system and its initial state:

$$
\theta(x)=T(x)-T_{0}
$$


Ultrasonic thermometers are a relatively new technology and as such can be quite expensive, e.g. IMS, Inc. sells an ultrasonic thermometer for between $\$ 60 \mathrm{~K}$ and $\$ 80 \mathrm{~K}$. This device may be able to detect temperature changes in up to 16 layers (depending on the similarity of the materials), however doing so, requires knowledge of the material makeup of the system being measured.

\subsection{Original Design Concept}

A design concept was proposed by the funding agency for this project (TARDEC) to back out material thermal properties using an in situ approach. The plan involved outfitting a vehicle with an array of temperature sensors and then thermally activating the surface of the armor using a heat source. After completion of the experiment(s), measured temperatures and heat fluxes would be provided to a code that couples the MuSES thermal solver (ThermoAnalytics, Inc.) with an optimization algorithm to determine the material properties, as illustrated Figure 2.1.
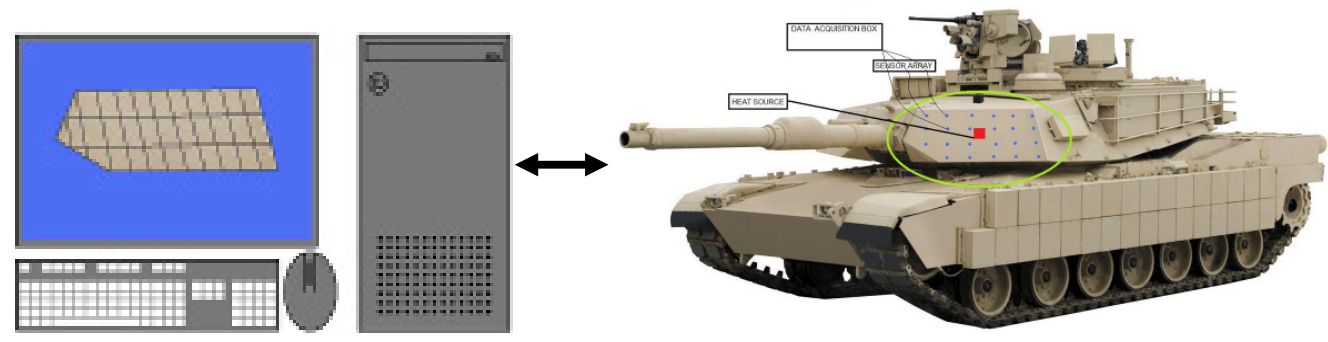

Figure 2.1 - Illustration of the original design concept test setup 


\section{Summary of original design concept:}

1. Heat source is applied to a surface

2. Array of thermocouples are applied to both front and back surfaces

3. Computer running LabView controls the heat source

4. LabView output data (temperatures and heat fluxes) are read in by a MuSES user routine

5. Iteratively run MUSES simulations while optimization code finds matching thermal resistance and capacitance 
Chapter 3 - Objective

\section{Chapter 3}

\section{Objective}

The goal for this research project was to develop a device and associated test methods that can be applied to military ground vehicle components in situ for the purpose of producing a set of thermal modeling properties. While a device that is able to accurately reverse engineer a vehicle component would be extremely valuable in its own right, it is important to note that the intent of the desired device behind this effort is to obtain a set of approximate property values that essentially produce accurate overall vehicle temperature predictions when performing thermal simulations of ground vehicles.

Advancement of the current techniques for material thermal properties measurement, from an in situ perspective, is beneficial to research scientists and engineers performing numerical simulation of systems consisting of unknown or unpublished material properties. By providing researchers with a tool to directly measure the thermal properties of the components of interest, they will no longer need to rely on estimates based on engineering judgment or require time consuming searches for information. The use of such a tool in industry will facilitate the rapid development of thermal models, thereby reducing project cost. Such a tool could also be beneficial to educators and students by providing a practical insight into the material properties of commonly encountered objects in everyday life. 


\section{Chapter 4}

\section{Methods}

\subsection{Basic Approach}

The solution approach for most thermal modeling problems is to predict a set of temperatures for an object of interest, given a set of known thermal properties, initial conditions, and boundary conditions. To accomplish this, conservation of energy is enforced by solving the heat equation at each location within the model. The governing equation in its most basic form (neglecting internal heat generation) can be expressed using two terms: i) A heat storage term for characterizing transients based on an initial state; and, ii) a conduction term for characterizing heat transfer within the object; i.e.,

$$
\rho c_{p} \frac{\partial T}{\partial t}=k \nabla^{2} T
$$

where $\rho$ is the density $\left(\mathrm{kg} / \mathrm{m}^{3}\right)$ of the material, $c_{p}$ is the specific heat of the material $(\mathrm{J} / \mathrm{kg} \cdot \mathrm{K}), \mathrm{T}$ is the temperature $(\mathrm{K}), \mathrm{t}$ is the time $(\mathrm{s})$, and $k$ is the conductivity of the material $(\mathrm{W} / \mathrm{m} \cdot \mathrm{K})$. The boundary condition for characterizing environmental heat transfer at the surface, $q_{e n v}^{\prime \prime}$, typically includes effects due to convection, radiation, and 
conduction; however, latent heat transfer (e.g. evaporation, condensation) involving phase change at the surface can also be included, when applicable:

$$
-k \nabla T=q_{e n v}^{\prime \prime}
$$

Rather than treating the temperatures as the unknowns as in typical thermal modeling applications, the basic approach behind material properties measurement is to treat the model parameters as the unknowns and solve for them by exciting the system using known, or measured, boundary conditions and the measured transient temperature response.

For layered materials, it can be shown from Equation 4.1 that the front side temperature and heat flux response is a function of the material properties of the constituent layers. For example, a generalized planar stack-up of equal thickness materials can be discretized into distinct temperature regions, which can be expressed using the following system of simultaneous equations:

$$
\begin{aligned}
& \Delta x \rho_{1} c_{p, 1} \frac{\left(T_{1}-T_{1}^{\prime}\right)}{\Delta t}=q_{\text {front }}^{\prime \prime}+\frac{k_{1}}{2 \Delta x}\left(T_{2}-T_{1}\right) \\
& \Delta x\left(\rho_{j} c_{p, j}+\rho_{j+1} c_{p, j+1}\right) \frac{\left(T_{i+1}-T_{i+1}^{\prime}\right)}{\Delta t}=\frac{k_{j}}{2 \Delta x}\left(T_{i}-T_{i+1}\right)+\frac{k_{j+1}}{2 \Delta x}\left(T_{i+2}-T_{i+1}\right) \\
& \Delta x \rho_{m} c_{p, m} \frac{\left(T_{n}-T_{n}^{\prime}\right)}{\Delta t}=\frac{k_{m}}{2 \Delta x}\left(T_{n-1}-T_{n}\right)+q_{b a c k}^{\prime \prime}
\end{aligned}
$$


where $j$ subscripts indicate the material layer, and $i$ subscripts indicate locations at which temperatures will be solved for (i.e., at the front and back, as well as at the interfaces between materials), and $T^{\prime}$ indicates the temperature from the previous time step. Each term in the equations has units of flux $\left(\mathrm{W} / \mathrm{m}^{2}\right)$, including the boundary conditions, $\dot{q}_{\text {front }}^{\prime \prime}$ and $\dot{q}_{b a c k}^{\prime \prime}$, which may also include an appropriate imposed heat source necessary to excite the system for property determination purposes.

\subsection{Approximation Using Effective Material Properties}

The feasibility of approximating the thermal response of an armor specimen (based on a representative CAV armor recipe) by using a single effective anisotropic material was examined to determine if a simple thermal model could be used to represent layered armor.

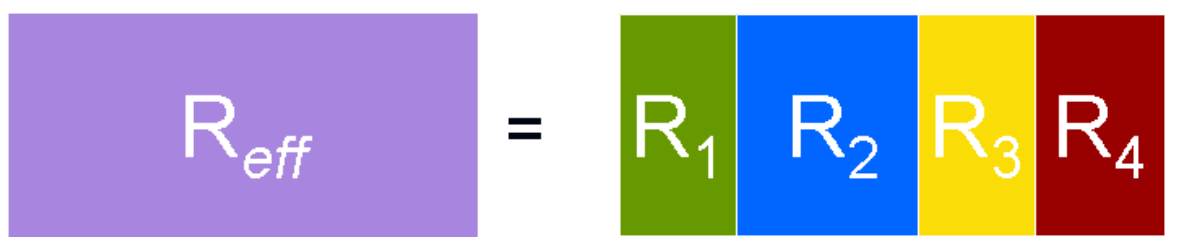

Figure 4.1 - Approximating armor thermal response using an effective resistance

Effective material properties can be constructed by summing up the individual resistances that appear in series as follows, 


$$
R_{e f f}=\sum_{i}^{n} L_{i} / k_{i}
$$

where $R_{\text {eff }}(\mathrm{K} / \mathrm{W})$ is the resulting effective resistance, and $L_{i}(\mathrm{~m})$ and $k_{i}(\mathrm{~W} / \mathrm{m} \cdot \mathrm{K})$ are the thickness and thermal conductivity of an individual layer making up the complete armor recipe, respectively. A similar procedure can be employed for obtaining the effective capacitance, $C_{\text {eff }}(\mathrm{J} / \mathrm{K})$,

$$
C_{e f f}=\sum_{i}^{n}\left(\rho V c_{p}\right)_{i}
$$

expressed here in terms of the density, $\rho\left(\mathrm{kg} / \mathrm{m}^{3}\right)$, volume, $\mathrm{V}\left(\mathrm{m}^{3}\right)$, and specific heat, $c_{p}$ $(\mathrm{J} / \mathrm{kg} \cdot \mathrm{K})$, of the individual layers.

A series of preliminary thermal modeling studies was performed to validate the approach of using a single effective anisotropic material to approximate an actual armor specimen. Two armor models were employed for this analysis; i) one based on a representative armor recipe; and ii) another based on a single effective anisotropic material. Both models were appropriately discretized through the thickness in order to accurately capture the transient temperature profile. For these analyses, a heat source was applied at the front surface and the temperature on the backside of the armor was logged over a period of simulation time (Figure 4.2). 


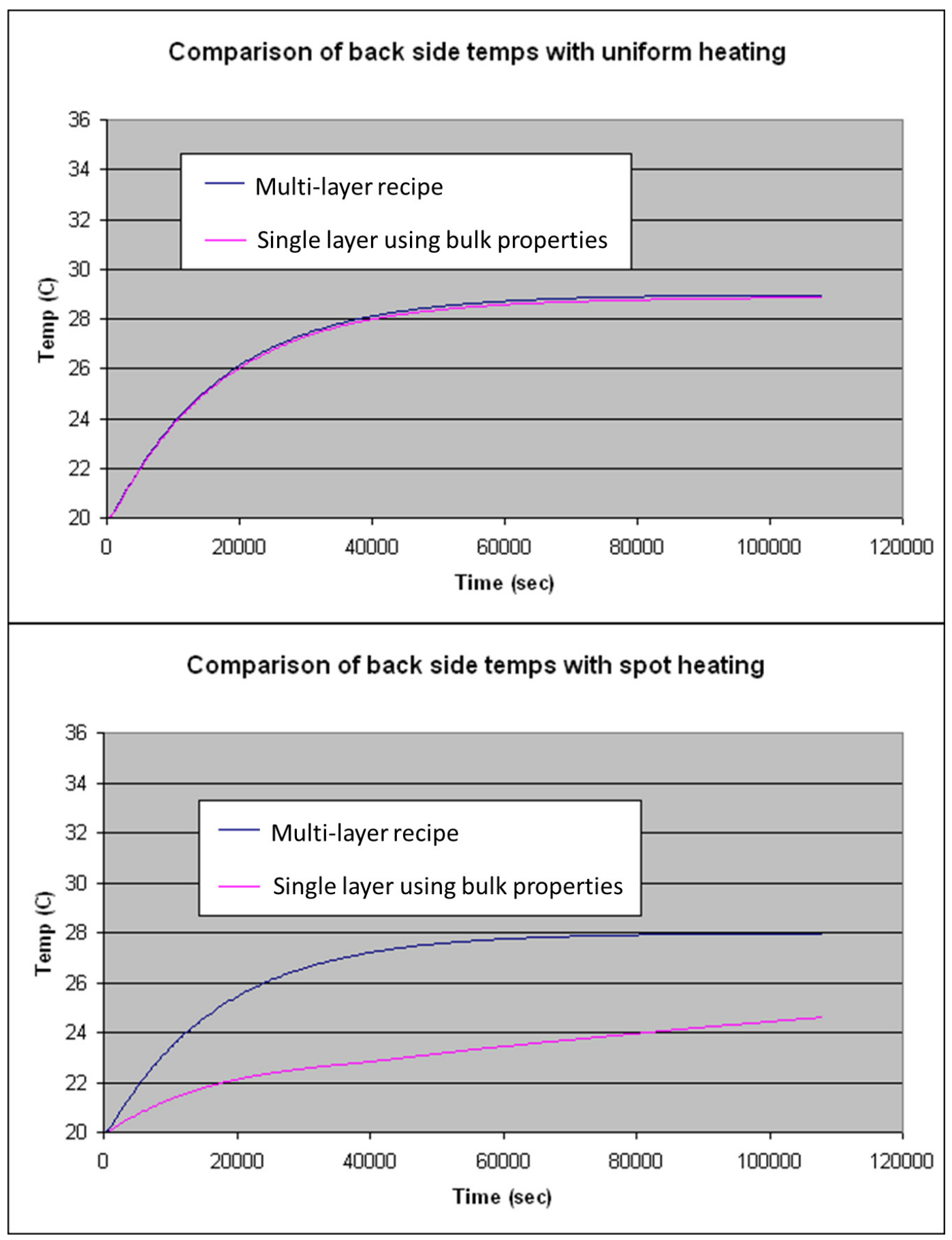

Figure 4.2 - Use of a single effective material to characterize a layered specimen 
Results from these preliminary thermal analyses indicate that using a single anisotropic material is not sufficient for characterizing armor plates when dealing with local spot heating. Therefore, it may be necessary to solve for an effective armor construction, that is, to have the thermal measurement device provide a resulting effective layered armor recipe consisting of at least two or three layers, in order to explicitly capture any anisotropic effects due to layering of armor materials.

\subsection{Accessibility to Armor Backside}

A visit to a military vehicle testing site containing a variety of armored vehicles provided an opportunity to examine the practicalities involved in performing actual armor thermal testing experiments. A hindrance to the feasibility of the proposed original concept

immediately became apparent: The inability to instrument the backside of an armor plate installed on a vehicle (Figure 4.3). 


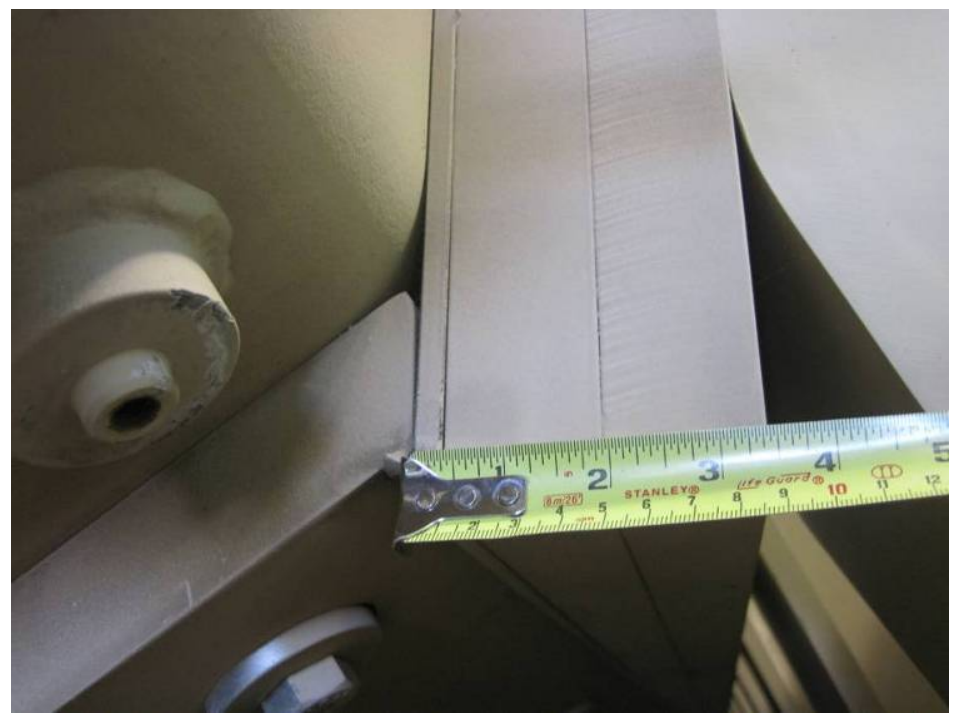

Figure 4.3 - Accessibility of Armor Backside

It follows that the testing procedure may undoubtedly require an approach that involves applying the heat source and measuring the temperature response on the same surface.

\subsection{Investigation of Heater/Armor Interaction}

An additional thermal simulation analysis was performed to gauge the quality of response information obtainable from careful instrumentation of the armor surface using an array of thermocouples (Figure 4.4). The purpose of this investigation was to ascertain the spatial variation of the surface heat diffusion from both a qualitative as well as a quantitative perspective. 

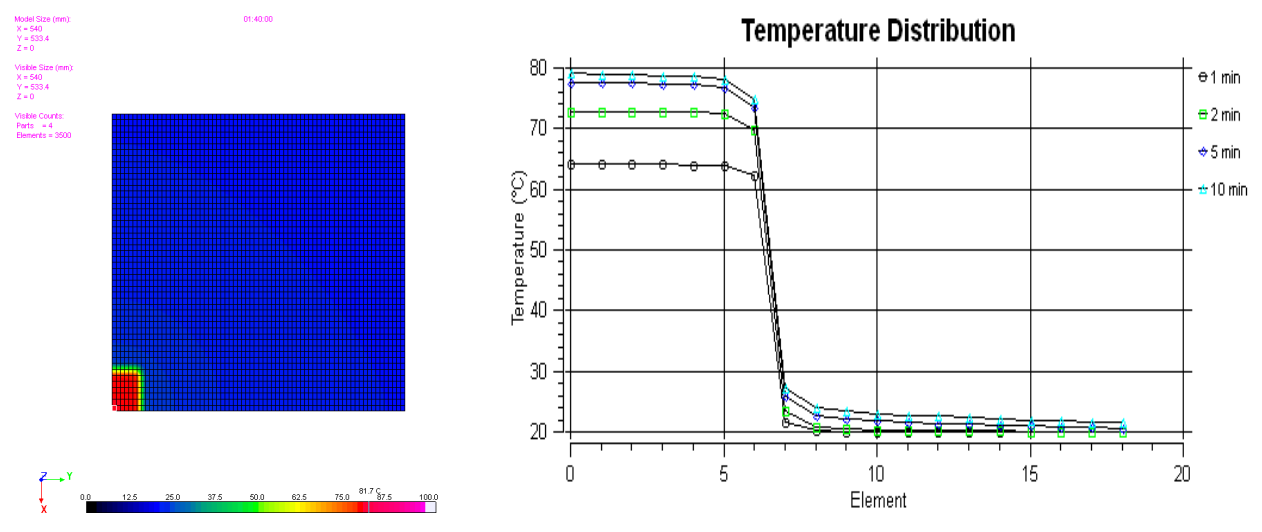

Figure 4.4 - Front side spatial variation

The results from these thermal simulations based on representative armor recipes indicate that the heat affected zone is essentially confined to the heater footprint. This conclusion was further confirmed by the results of physical measurements on actual armor that was performed at a military vehicle testing facility early on in the project. These findings indicate that the final design of the measurement device may only require a single heat flux sensor and/or thermocouple positioned directly below the heater element. 


\section{Summary of Preliminary Technical Hurdles:}

1. Difficult or impossible to access backside of armor

a) Will need to apply heat and measure temperature response from same side

b) Backside boundary conditions will be difficult to characterize

2. Minimal spatial temperature variation on front side

a) Limited to only one relevant response (temperature) measurement

3. Very little information on actual armor recipes and material properties in published literature.

a) Validity of measurements based on actual armor will be difficult to assess

\subsubsection{Development of an Ultrasound-based Property Measurement Device}

Constructing an ultrasonic thermometer capable of determining temperature changes within a multilayer specimen requires coupling an extremely high-precision data acquisition system with a sophisticated signal processing framework. A set of various sized transducers capable of emitting and receiving a wide range of frequencies would also be required.

Ultrasonic flaw detection devices tend to be much more versatile than their more common thickness gage counterparts. These devices typically allow direct access to the signal of interest, providing estimations of time of flight measurements from layer to layer as determined by embedded signal processing techniques. Subsequently, ultrasonic 
flaw detection devices are more expensive than thickness gauges: For example, a MasterScan ultrasonic flaw detector from Sonatest costs about $\$ 8,500$.

Preliminary calculations (confirmed by physical testing on actual armor with an ultrasonic technician on-site) indicated that the level of precision provided by these devices is not sufficient for internal temperature measurement within the relatively tight range of temperatures necessary for thermal property measurement. The development of a more precise data acquisition system may explain the prohibitively high cost of the ultrasonic thermometers provided by their manufacturers.

\section{Ultrasound Technical Hurdles}

Ultrasonic temperature measurement requires knowledge of layer materials and thicknesses

- Layer transition from low to high impedance could cause a reflection of most of the ultrasonic wave, reducing ability to measure material properties beyond this layer

- Layers beyond any air or rubber layers would be difficult to characterize

- Excessive noise could make signals and echoes difficult to process the precision required for thermal property measurement

- Material properties needed for relating temperature to sound speed for commonly used armor materials are difficult to obtain 


\subsection{A Thermal Measurement Only Approach}

Considering the compounding series of difficulties associated with the ultrasonic approach, the extent and nature of the properties that can be obtained from using a pure thermal approach (that is, when the experiment is limited to applying heat and measuring temperature from same side of an armor component) was investigated.

It can be shown from an analytic solution involving a semi-infinitely thick slab exposed to a surface heat flux (i.e. the semi-infinite solid solution [17]) that a relatively simple closed form equation can be obtained relating material properties of interest to surface heat flux and temperature,

$$
k \rho c_{p}=\frac{4 t}{\pi}\left(\frac{q_{s}^{\prime \prime}}{T_{s}-T_{i}}\right)^{2}
$$

where $t$ is the elapsed time (s), $q_{s}^{\prime \prime}$ is the heat flux imposed at the surface, $T$ is the temperature (K), and the subscripts $s$ and $i$ denote "surface" and "initial," respectively. The resulting material properties are combined to form a single value representing the product of conductivity, density, and specific heat, or from an analogous electrical perspective, the quotient $\mathrm{C} / \mathrm{R}$, where $\mathrm{C}$ is the thermal capacitance $(\mathrm{J} / \mathrm{kg} \cdot \mathrm{K})$ and $\mathrm{R}$ is the thermal resistance $\left(\mathrm{m}^{2} \cdot \mathrm{K} / \mathrm{W}\right)$. Despite the fact that the desired material properties are 
expressed as a product, this result is still useful given that the heat capacity tends to be similar for many armor materials.

A simple MuSES model of a flat steel plate was constructed to determine the validity of applying the semi-infinite solid solution to a non-semi-infinite slab (such as an armor plate) to obtain thermal properties. The conductivity of the armor material was solved for based on values of heat flux, temperature, elapsed time, given an exact value for the product of heat capacity and density. The results of this experiment are shown in Figure 4.5 .

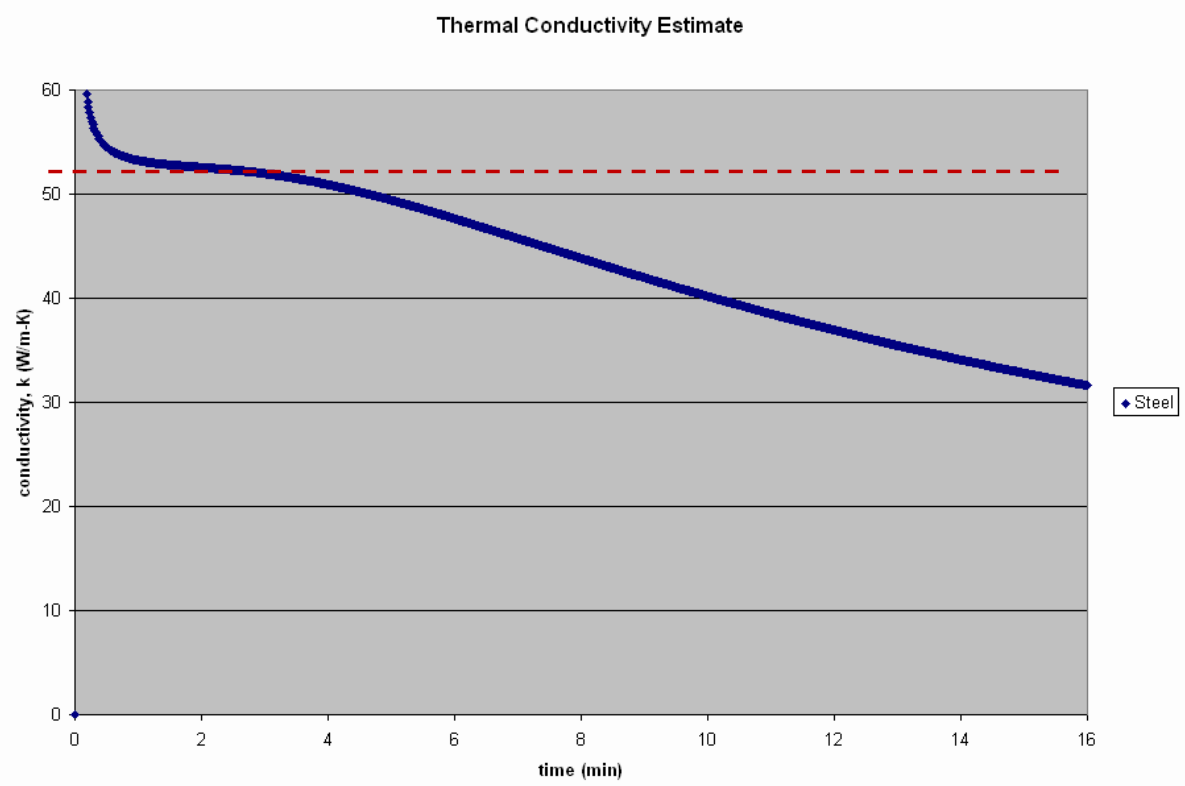

Figure 4.5 - Estimation of conductivity using the semi-infinite solid approximation

It was found that solution of the semi-infinite solid equation results in an accurate prediction of the assigned conductivity value $(52.0 \mathrm{~W} / \mathrm{m}-\mathrm{K})$ when confining the 
experimental data to a window positioned near the beginning of the experiment. A plateau in the estimate appears at the location of the correct conductivity value which, with appropriate signal processing, may be used to determine a convergence criterion. The analytical estimate begins to drop off at the same point in time at which the surface heat flux begins to significantly affect the backside temperature. At this point in the experiment, the model no longer behaves like a semi-infinite solid. In other words, the semi-infinite solid approximation begins to break down as the backside boundary condition begins to affect the surface temperature response.

This investigation provides an indication that the surface temperature response may help characterize the backside boundary conditions, or at least at a minimum, provide the analysis with a stopping point trigger that bounds the window of valid experimental data needed to avoid the need to characterize the backside boundary conditions.

\subsection{Optimization-MuSES coupling}

Although the closed-form solution to the semi-infinite solid problem appears to indicate that it is only the combination of the capacitance and resistance that can be obtained from knowledge of the elapsed time, surface temperature and heat flux, an effort was undertaken to attempt to solve for these material properties independently of one another using a combination of a thermal solver and an optimization code, i.e. MuSES and Mode FRONTIER (Esteco), respectively. 
A test model was constructed involving a homogeneous sheet of material consisting of mild steel $\left(k=52.019(\mathrm{~W} / \mathrm{m} \cdot \mathrm{K}), c_{p}=460.967(\mathrm{~J} / \mathrm{kg} \cdot \mathrm{K}), \rho=7768.98\left(\mathrm{~kg} / \mathrm{m}^{3}\right)\right)$. To improve accuracy, the sheet was subdivided into multiple thermal nodes, equally spaced through the thickness from the front to the backside. The surface was exposed to a constant heat flux for the duration of the 30 minutes of simulation time. For this simple case, the heat transfer was assumed to flow in only one direction, from front to back, and an adiabatic boundary was assumed in all other directions. A single front side surface temperature was logged at every minute during the simulation. This data was written out to an ASCII file so that it could be subsequently read in by an optimizer.

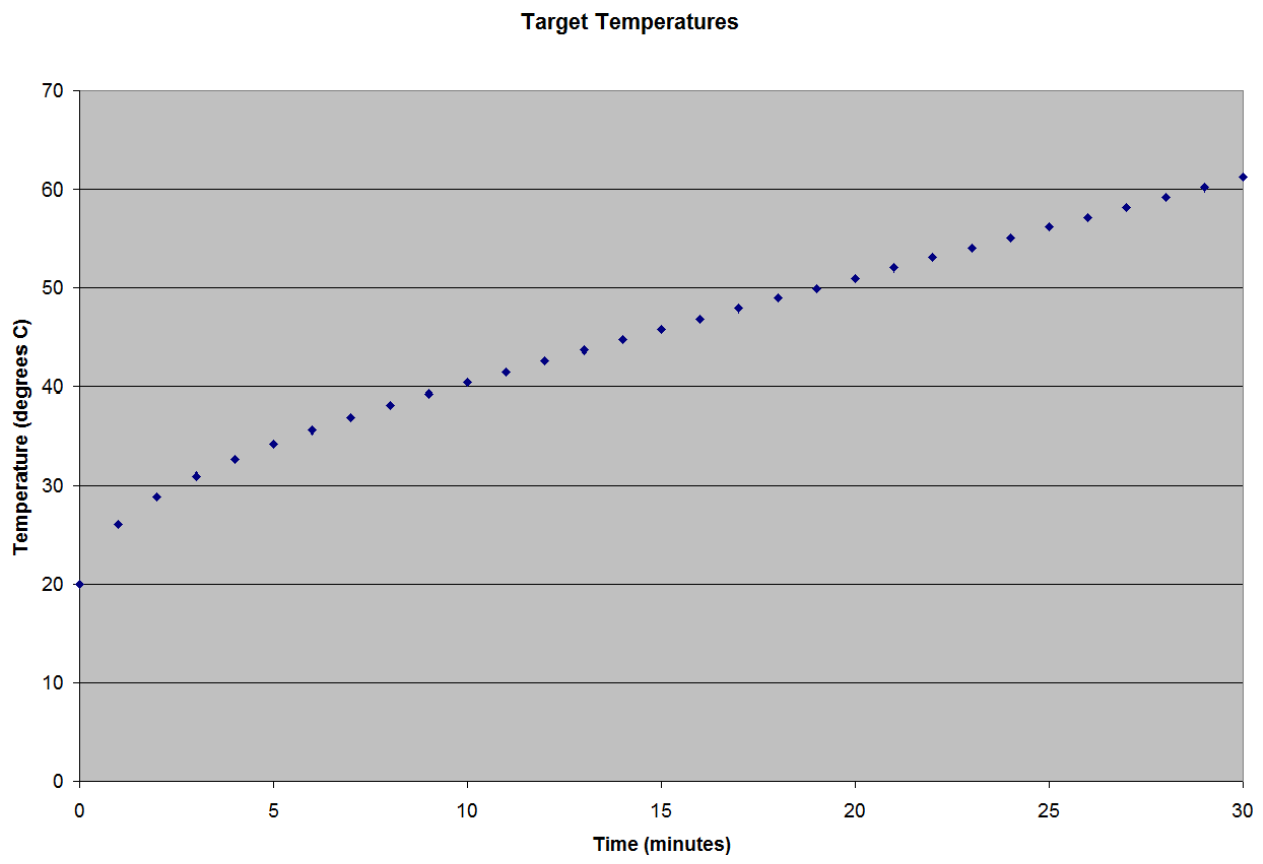

Figure 4.6 - Target temperatures used by the optimizer 
The Mode FRONTIER optimizer was used to extract the temperatures from the thermal solver's ASCII output file and find both thermal resistance and heat capacity simultaneously and independently of one another through a series of controlled thermal simulations. In order to gauge the progress of the optimizer during the solution by monitoring the thermal properties in terms of conductivity and specific heat, the same thickness and density that were supplied to the Mode FRONTIER control model were also supplied to the optimized model; these values were held constant in all cases. The Mode FRONTIER optimizer was able to converge on both an accurate as well as precise result for the conductivity value $\left(\mathrm{k}_{\text {effective }}=52.019 \mathrm{~W} / \mathrm{m} \cdot \mathrm{K}\right)$ and specific heat value $(460.967 \mathrm{~J} / \mathrm{kg} \cdot \mathrm{K})$ after approximately 170 iterations (Figure 4.7$)$.

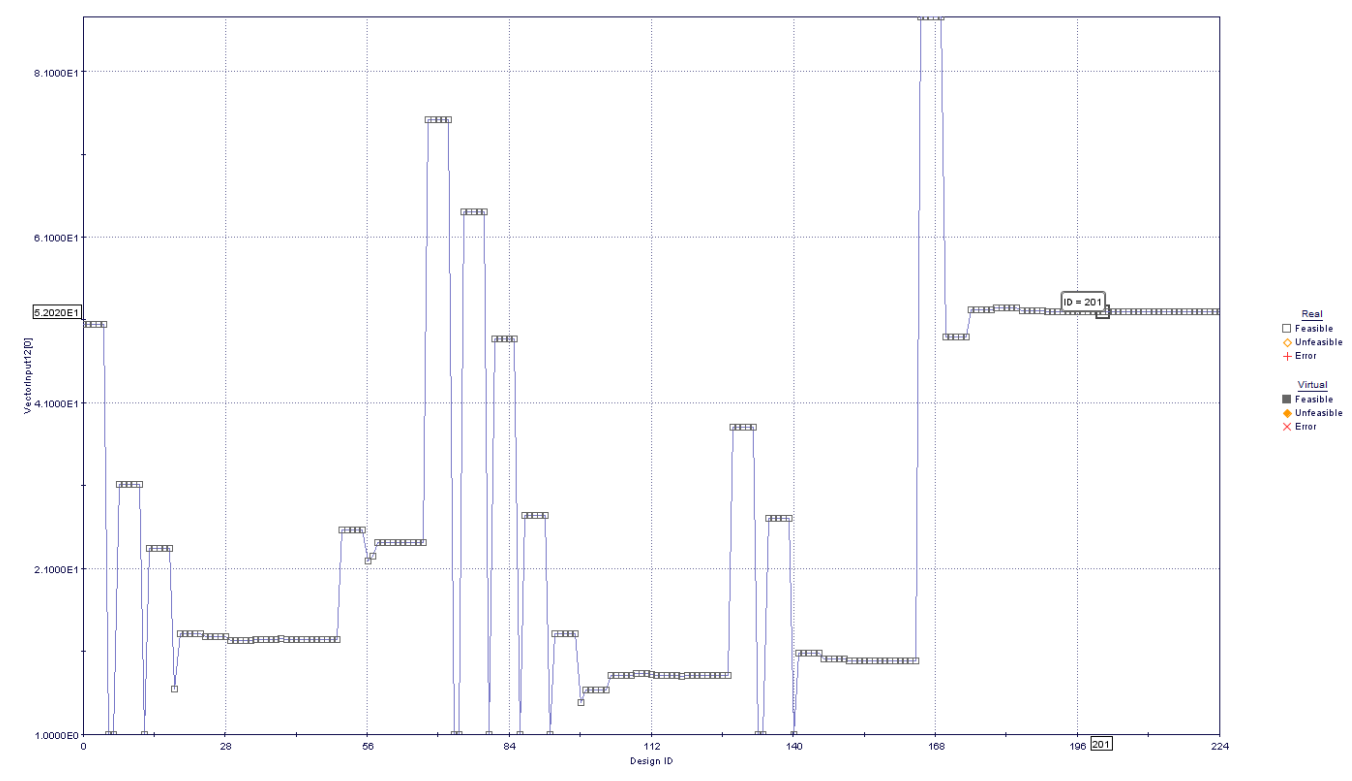

Figure 4.7 - ModeFRONTIER optimization convergence 
This same experiment was also performed with an internally developed Nelder-Mead based optimizer and yielded similar results.

\subsection{Time Varying Inputs}

A series of simulations based on actual armor recipes was performed to characterize the time window available for capturing meaningful front side temperature responses when using a step input function. This window is defined as the elapsed time between the start of the experiment and the point at which the heat input has a chance to significantly influence the back side temperature.

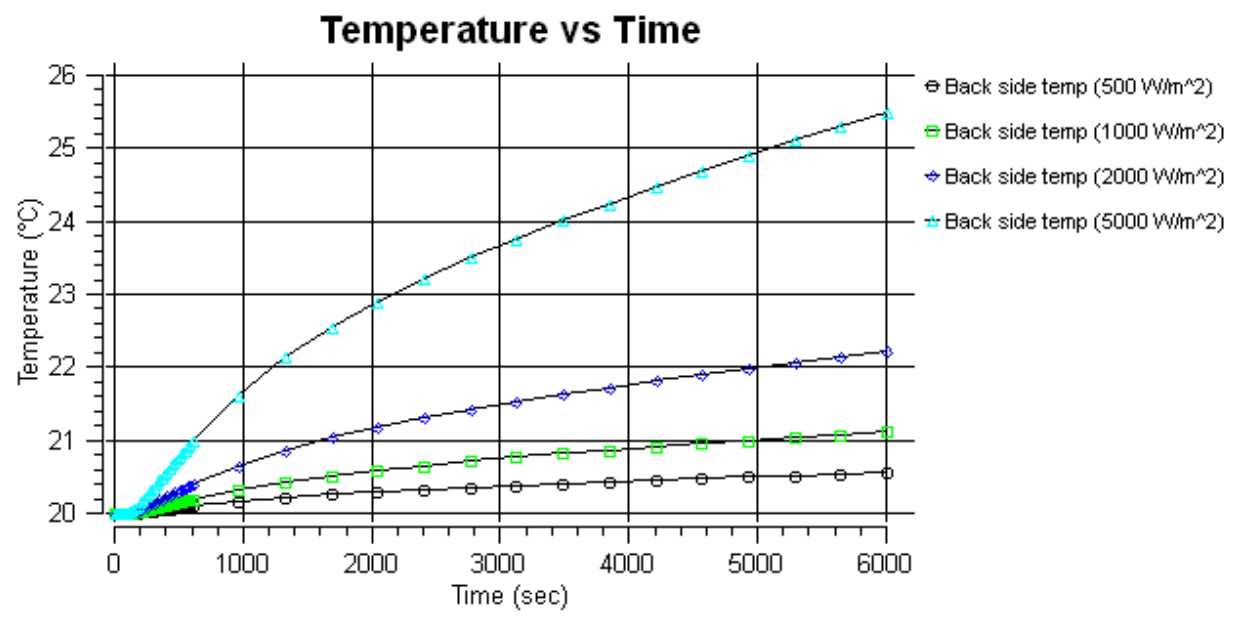

Figure 4.8 - Assessing the time until the backside temperature is affected

Imposing a constant heat flux on the surface can result in a significant backside temperature deviation after only 'a few' minutes, depending on the magnitude of the heat 
Chapter 4 - Methods

flux supplied. This sets a very short time period for which the 'insulated back side' assumption is valid. However, if a periodic heat flux were imposed on the surface, which could remove the same amount of heat as injected, it is possible to keep the back surface temperature from deviating from its initial state. Furthermore, a periodic (sinusoidal) heat flux would provide a testing course that would expose the test object to a wide range of controlled thermal states. High frequencies would heat primarily the outer layer(s) closer to the front surface, while the low frequencies would also excite the back layers.

A detailed investigation was undertaken to examine the front surface temperature response due to the thermal excitation by a sinusoidal heat flux input. The thermal response was examined for various frequencies and amplitudes as well as for various material stack-ups. To begin with, a simple two-layer configuration was put together in which the front surface layer's material properties were held constant, and the back layer's material properties were varied, resulting in models consisting of the following: Steel over copper, steel over rubber, and steel over steel. A series of sinusoidal heat fluxes was applied to each configuration until the object reached a sustained thermal state for each particular frequency and amplitude. The results of each simulation were then combined to form a plot of the frequency response function for each configuration (Figure 4.9 and Figure 4.10). 
Chapter 4 - Methods

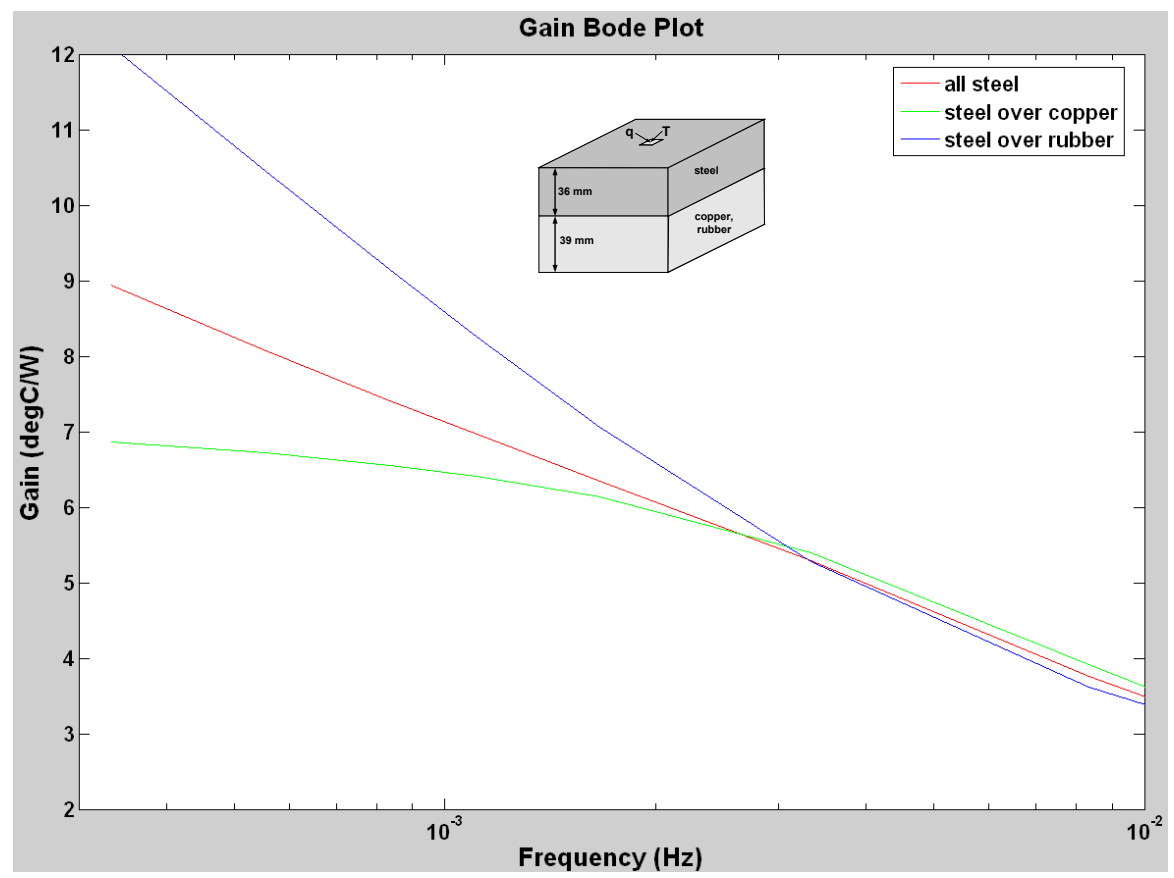

Figure 4.9 - Gain response of steel cover layer configuration

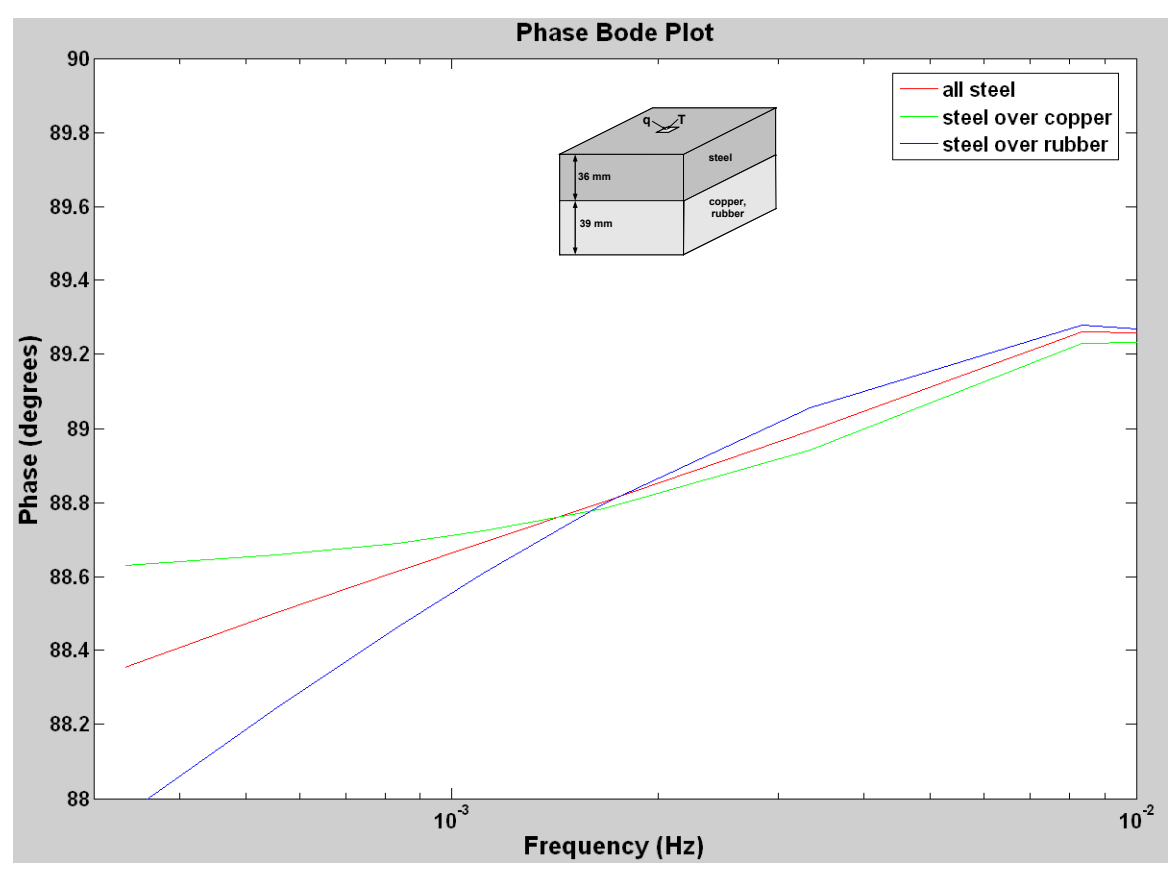

Figure 4.10 - Phase delay of steel cover layer configuration 
The gain (defined as the output divided by the input, $\Delta T_{s} / \Delta q_{s}^{\prime \prime}$ ) and the phase delay of the temperature response relative to the imposed heat flux can substantially differ in each individual simulation, depending on both the material stack-up and the excitation frequency. This behavior is significant since it indicates that the surface temperature response is dependent on the material properties of the layers within the object. As can be seen in the frequency response plots, the amplitude and phase tend to the same value for higher frequencies (short periods as shown in Figure 4.11) and then begin to diverge as the frequency of the input signal is decreased. 
Chapter 4 - Methods
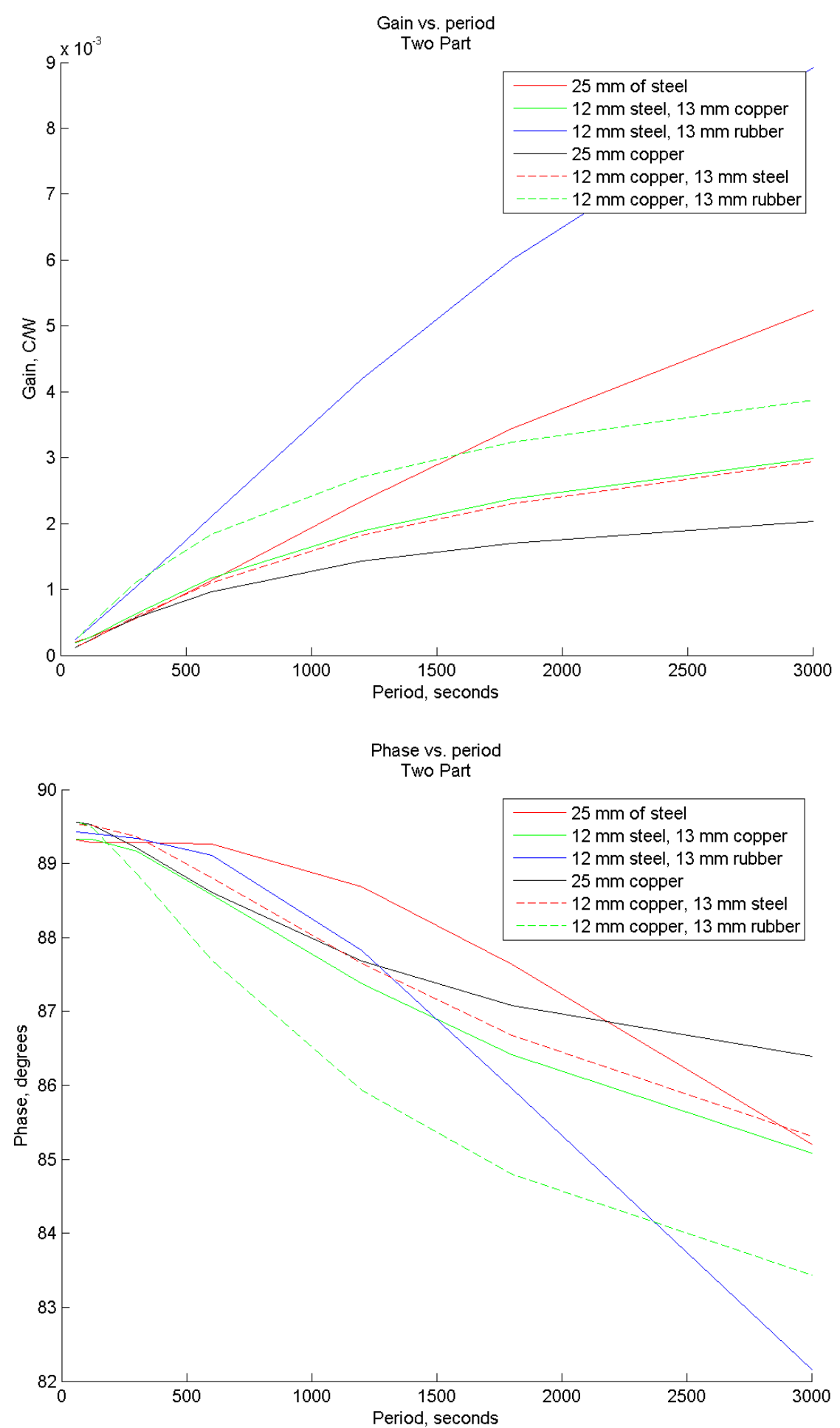

Figure 4.11 - Frequency response when front cover layer is varied 
The higher frequencies excite mainly the top surface layer, which subsequently dominates the frequency response of the entire layered material. Conversely, the lower frequency inputs also excite the deeper layers, resulting in a different response for each layer configuration. Given these results, it follows that it may be possible to separate the effective layer properties by careful excitation and examination of the response at different frequencies.

This study was extended to analyze differences in frequency response for material stackups in which the front layer material was varied. This analysis also showed significant differences in frequency response for each configuration.

A set of frequency response functions was also obtained from simulations consisting of material stack-up configurations of representative armor recipes. Although a broad overlap was found to exist in the materials used in each of the armor recipes, the frequency response of the materials was found to be different for each configuration. The frequency response functions are also very different depending on the side being examined, further confirming the fact that the order of layers in the armor stack up is significant in terms of heat diffusion through the system. 


\subsection{Characterization of Individual Effective Layers}

The following method was developed for determining an operative layered material construction that is thermally representative of a corresponding armor plate for which thermal modeling parameters are desired: i) Perform a series of tests with sinusoidal heat flux inputs (i.e. one frequency per test) and measure the surface temperature response; ii) Find the effective layer construction by optimizing each layer of a thermal model. The thermal model will consist of the same number of layers as there are frequencies for which test data is available; the optimization routine would find the thermal properties of each layer separately.

For example, given experimental data based on three input frequencies, three optimizations could be performed to obtain the thermal resistance and heat capacity of each individual effective layer. With proper selection of frequencies, the high frequency test data would be used to characterize the front effective layer; the medium frequency would be used to characterize the middle effective layer; and the low frequency would be used to characterize the back effective layer. 


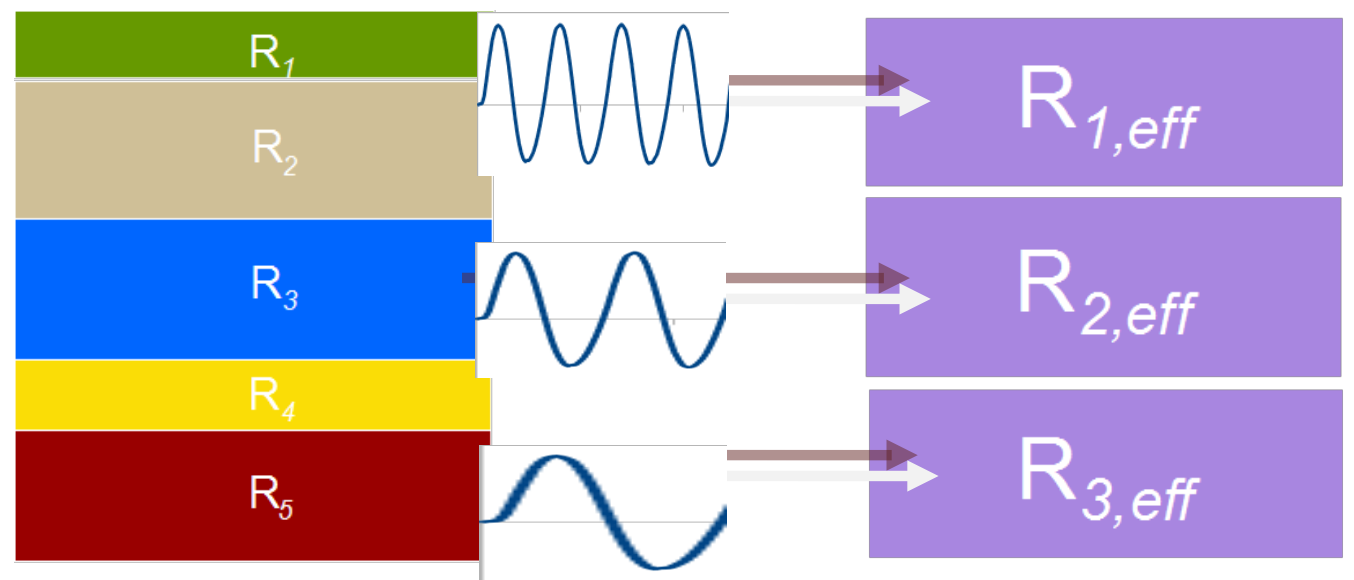

Actual Armor Recipe

Recipe Ottained from Measurement Device

Figure 4.12 - Characterization of individual effective layers

The optimization proceeds until the simulated armor's frequency response converges upon the frequency response based on the test data. Should convergence not be reached, it would be an indication that insufficient measurement data is available to characterize the armor, requiring additional experiments to be performed at other frequencies before an acceptable operative armor recipe is achieved.

\subsection{Boundary and Initial Condition Sensitivity}

A trade study was performed that involved successively running the thermal model using sinusoidal heat fluxes and adjusting the model inputs to find the sensitivity to various parameters. 
Chapter 4 - Methods

With respect to boundary conditions modeling, it was found that small radiation and convection inaccuracies have little effect on the temperature response underneath the heater where the measurement is taken for determining the material properties. This is because of the relatively high thermal resistance of the surroundings as compared to the high conductance within the solid material. For thermally resistive specimens (e.g. HDPE), the thermal wave does not propagate very far from the heater footprint, allowing the extended portions of the material sample to essentially remain at their initial state. This situation results in virtually an adiabatic condition at the boundary for the duration of the experiment. For thermally conductive samples the heat transferred from the heater is quickly diffused into the specimen, resulting in a reduced rise in temperature underneath the heater as compared to the high temperatures experienced with thermally resistive samples with similar heat inputs. Although the specimen portions far from the heater do experience a small rise in temperature due to this heat diffusion, the resulting potential difference between the surface of the specimen and the surroundings is also correspondingly small, resulting in a small increase in heat transfer from the sample to the surroundings. Therefore small inaccuracies in boundary condition characterization, while slightly more significant for thermally conductive specimens, have a negligible effect on the results of the property determination.

Given that the temperature response of highly conductive materials is reduced under the heating element, small thermocouple calibration errors have the potential to affect the optimizer's solution convergence. This can be further compounded by inaccuracies in 
initial condition characterization of the test specimen. In order to address this issue, a slope-only comparison approach was investigated for use within the optimizer cost function, given that preliminary experiments have shown that it is the amplitude and phase lag of the temperature under sinusoidal excitation that is affected by the material properties of a specimen rather than in its absolute temperature. The following fourth order central difference approximation was therefore used to characterize the slope of the transient temperature response:

$$
f^{\prime}\left(x_{i}\right)=\frac{f\left(x_{i-2}\right)-8 f\left(x_{i-1}\right)+8 f\left(x_{i+1}\right)-f\left(x_{i+2}\right)}{12 h}
$$

\subsection{Mesh Sensitivity Analysis}

A mesh sensitivity analysis was performed based on actual temperatures and heat fluxes obtained from measurement experiments. A preliminary low density mesh was developed to take advantage of the inherent symmetry associated with both the physical layout and the thermal wave propagation. This mesh represents one of the quadrants of the test setup, reducing the mesh size to a quarter of the full experimental setup (Figure 4.13). 

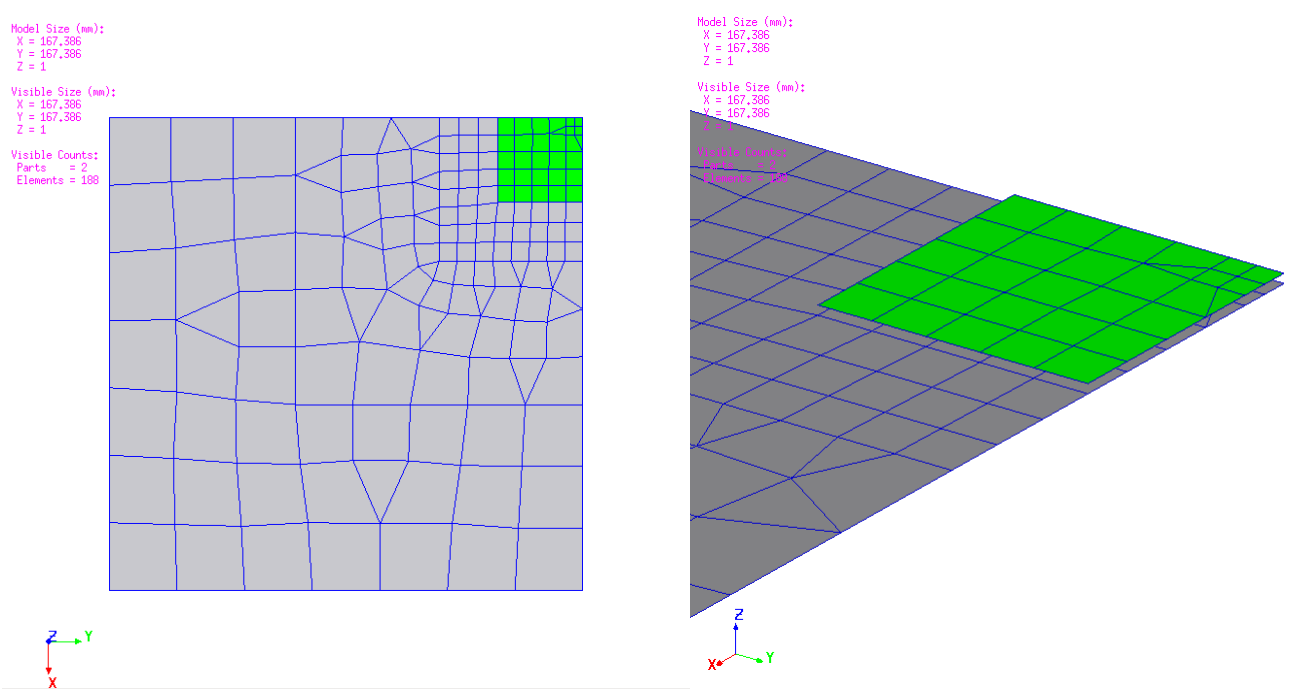

Figure 4.13- Coarse "quadrant" mesh with close-up of heater geometry

The effective $\mathrm{x}$ and $\mathrm{y}$ dimensions of both the heater and the armor are cut in half, with the heater positioned in one of the corners rather than in the center.

This mesh was further simplified by taking advantage of the remaining symmetry along the diagonal (Figure 4.14). The thermal results obtained from this simplification were found to be consistent with results obtained using the full quadrant mesh. 


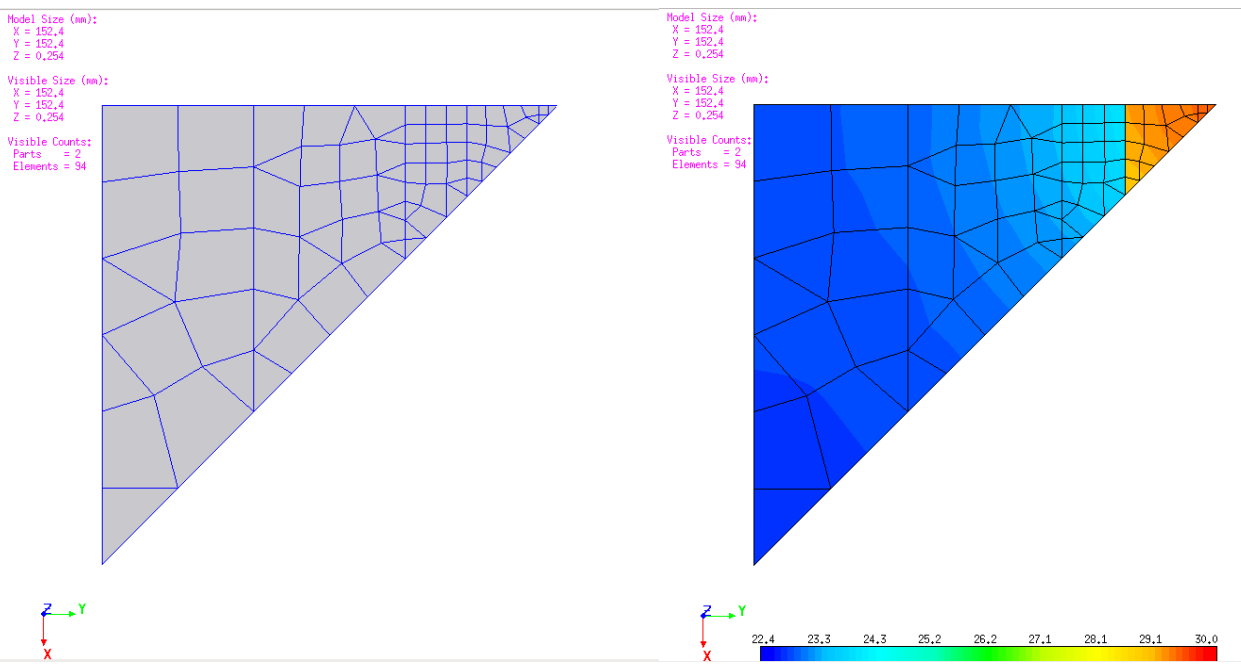

Figure 4.14- The half quadrant mesh takes advantage of symmetry to reduce mesh size

Although this mesh is sufficient for modeling thermally resistive specimens (e.g. HDPE), it is not adequate for the accuracy required for determining the thermal properties of thermally conductive specimens (e.g. aluminum). A subsequent effort was made to further increase the mesh density, especially in the area directly under the heater where the largest magnitude thermal gradients can occur (Figure 4.15).
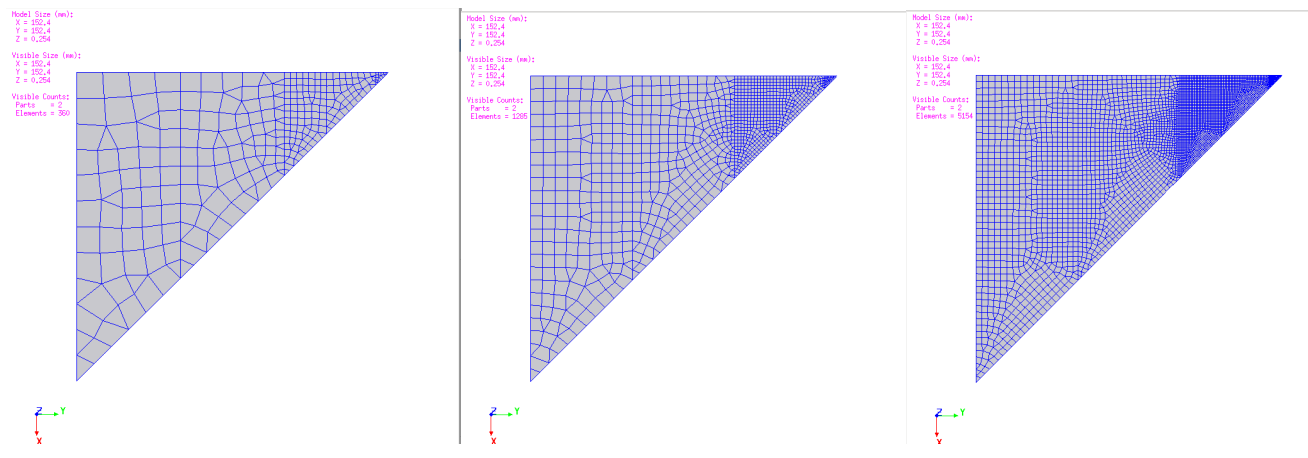

Figure 4.15 - Mesh refinement needed to capture transients in highly conductive specimens 
The finely discretized graded mesh reduces the element size in the corner (where the thermal couple is located) to approximately $1 / 3$ of a $\mathrm{mm}$. Due to the exhaustive number of elements, the thermal simulation time required to solve this mesh on a standard desktop or laptop computer is too lengthy to be practical, especially when considering the numerous iterative thermal solutions required for the purposes of property determination.

A compromise mesh was therefore constructed that retains the fine discretization under the heater, but provides the coarseness in the areas far off to aid in the reduction of thermal solution time (Figure 4.16). This mesh provides essentially the same accuracy as the most discretized mesh constructed for this analysis.

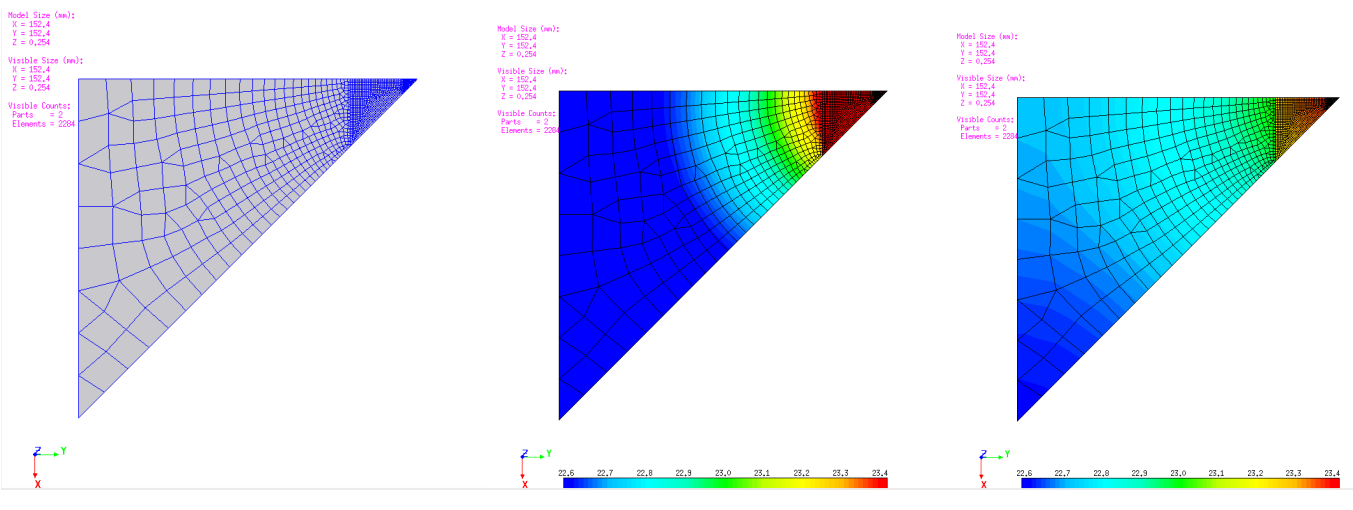

Figure 4.16 - Graded "compromise" mesh 


\subsection{Material Property Testing}

Testing armor samples as described above requires the input of a sinusoidal heat flux onto a surface resulting in a temperature response that oscillates about its initial temperature. The heat flux generating device must be carefully controlled so as to apply and remove heat at various stable frequencies. One such device, a thermoelectric cooler, is able to perform this function and is available as an off-the-shelf product.

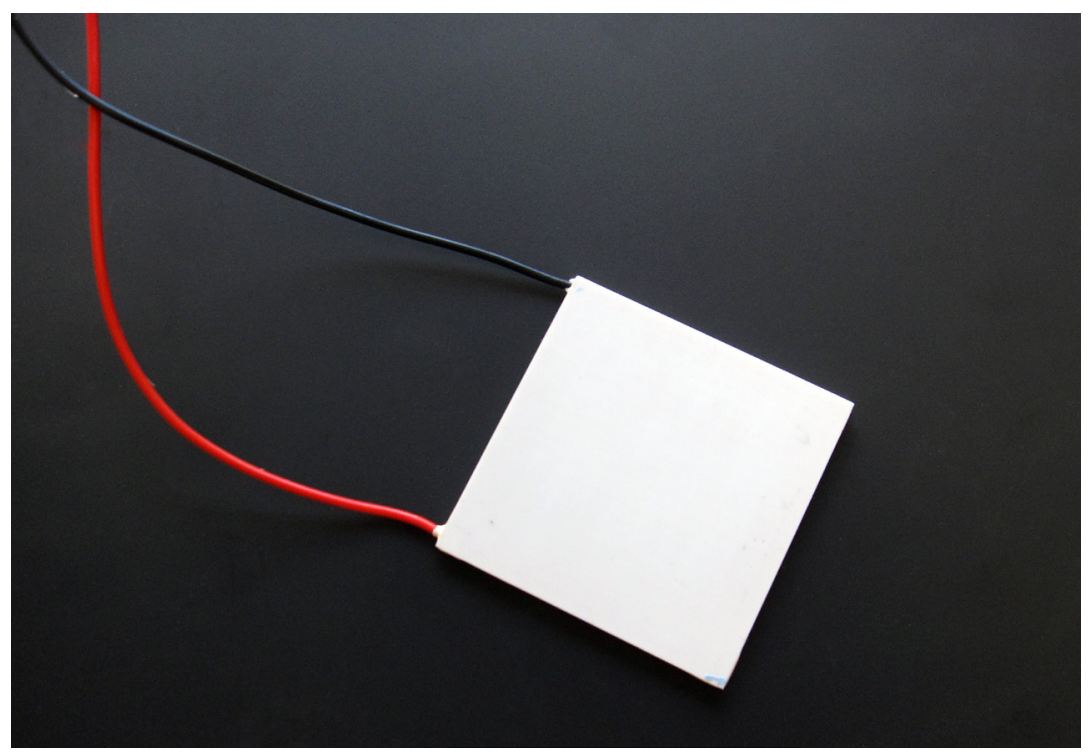

Figure 4.17- Example of a thermoelectric (Peltier) module

Thermoelectric devices are designed to directly convert a voltage difference into a temperature difference and vice versa. This phenomenon is known as the thermoelectric effect and results from the diffusion of charged carriers in the material in a direction relative to an applied temperature gradient. In addition to being able to provide heating 
and cooling, this effect has also been exploited by engineers to generate electricity and measure temperature differences within an object.

A high power thermoelectric heater/cooler (CP-200HT-TT) and associated controller (TC-36-25-RS485) were obtained to verify the practical feasibility of operating a thermoelectric device in a sinusoidal fashion. This thermoelectric device is the highest power device manufactured by TE Technology, Inc. (Traverse City, MI) with a capacity rating of 200 Watts at a zero degree temperature difference. An experiment using a function generator to provide a reference temperature was successfully completed. Using a 1" thick aluminum test specimen resulted in measureable sinusoidal temperature oscillations with periods of less than 2 minutes (Figure 4.18).

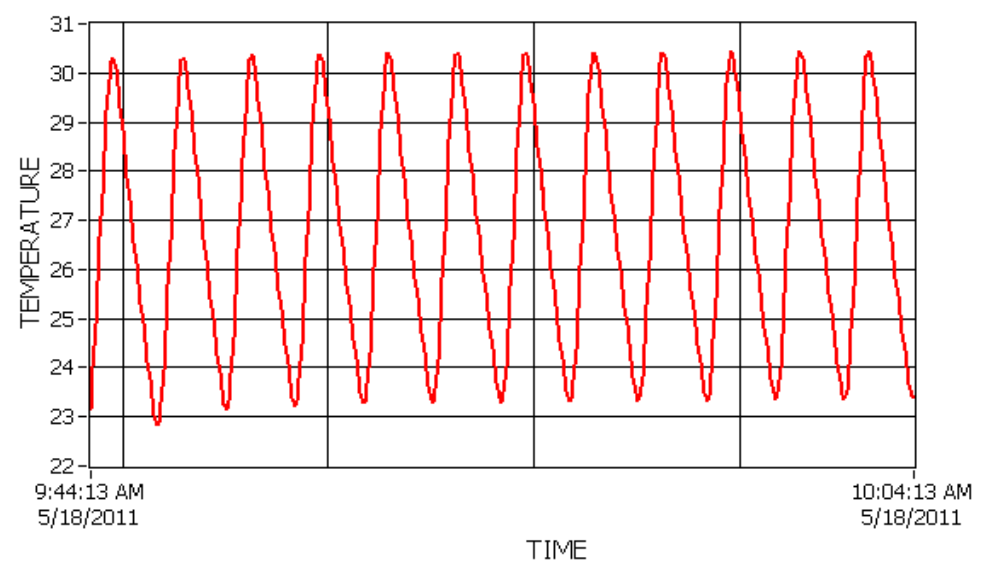

Figure 4.18- Sinusoidal operation of a thermoelectric device using temperature control 
Rather than using the thermoelectric plate temperature for control, it is more desirable to simply control the input current and voltage and measure the resulting the heat flux imposed onto the armor and the subsequent temperature response of the armor surface. For such an application, a single thin foil flexible heat flux sensor with an embedded thermocouple can be sandwiched between the thermoelectric heater/cooler plate and armor. These devices can be ordered to size, are reasonably priced, and can be glued on to a surface of interest.

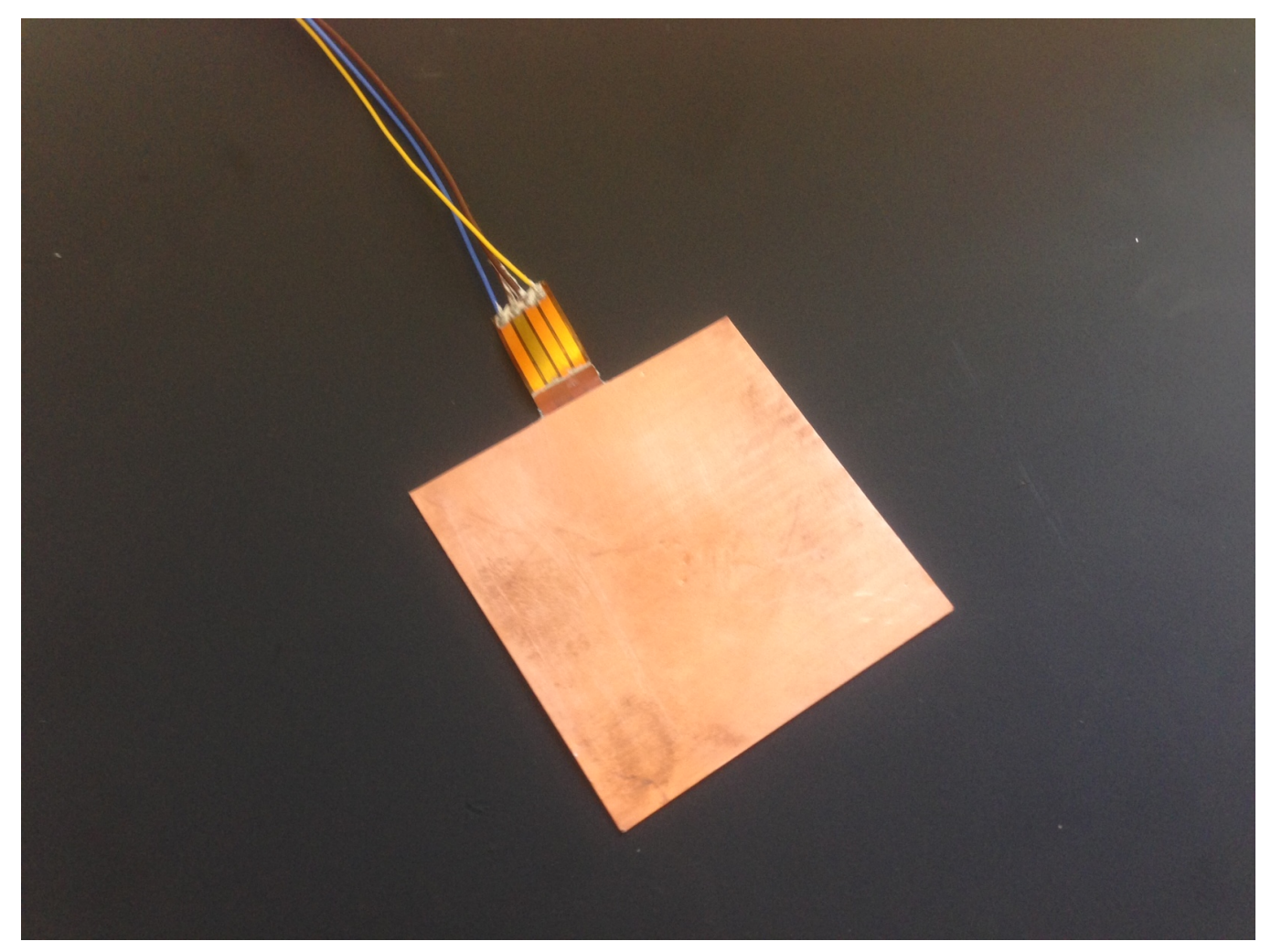

Figure 4.19- Example of a thin film heat flux sensor 
For example, Captec Enterprise manufactures a line of thin foil heat flux sensors that are $0.4 \mathrm{~mm}$ thick, have a response time of 0.3 seconds, and work within an operating range of -180 to $200{ }^{\circ} \mathrm{C}$. The manufacturer also provides a service to custom design a heat flux sensor to fit an area equal to the dimensions of the thermoelectric prototype device (216 $\mathrm{mm} \times 161 \mathrm{~mm}$ ). The total price for such a sensor is between $\$ 1000$ and $\$ 1500$ each. 


\section{Chapter 5}

\section{Development of a Property Measurement Device}

\subsection{Preliminary Proof-of-Concept Prototype}

A measurement device prototype was developed by Motolla, LLC to produce a sinusoidal heat input at a user-specified period so that the heat flux and temperature can be measured at the interface between the thermoelectric device and the test sample (Figure 5.1). The device was capable of recording the heat flux and temperature along with the time stamp with measurements taken at one second increments.

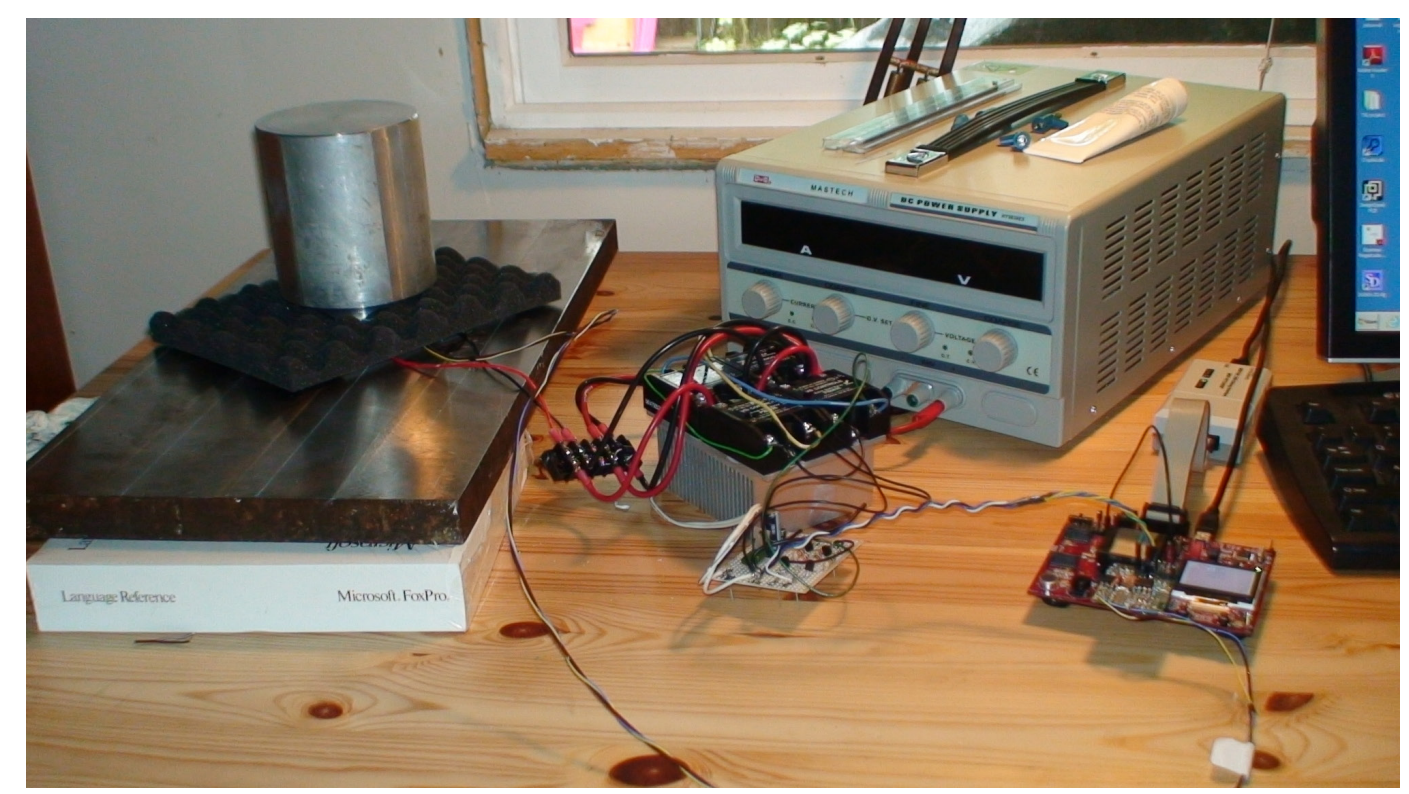

Figure 5.1 - Preliminary Measurement Device Prototype 
The prototype device consists of a TI MSP-EXP430F5438 test board with an embedded MSP430F5438 microprocessor (Figure 5.2). The $\mathrm{C} / \mathrm{C}++$ based control and processing code can be uploaded to the microprocessor firmware using a special MSP-FETU430IF programming pod.

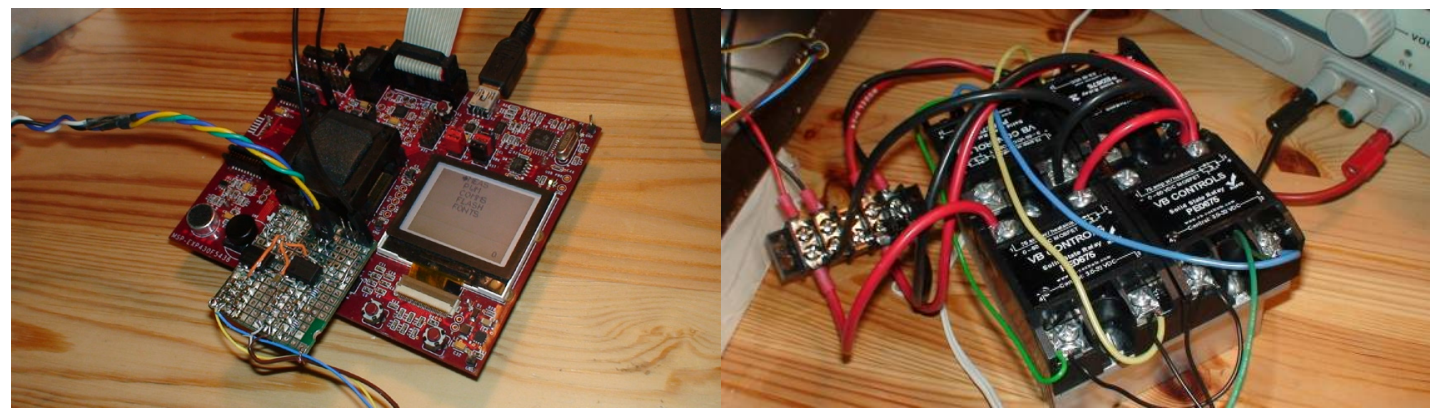

Figure 5.2 - TI MSP-EXP430F5438 test board with custom-built signal conditioner and H-bridge needed to drive sinusoidal operation of thermoelectric device

The device employs a pulse-width-modulation (PWM) technique to drive the necessary sinusoidal operation of the thermoelectric module. The average value of the input signal to the thermoelectric module is controlled by switching the power between on and off states. A custom built H-bridge component was developed to convert the pulse-widthmodulated sinusoidal signal from the testing board in order to drive a high-power thermoelectric unit. In addition, a custom-built interface board consisting of op-amps and filters provides conditioning of the heat flux sensor and thermocouple signals and removes electrical noise from $\mathrm{H}$-bridge operation prior to analog-to-digital conversion in the testing board. 
Chapter 5 - Development of a Property Measurement Device

\subsection{Thermoelectric Device and Heat Flux Sensor}

In order to obtain useful data for property determination, the device must have a heating element that is capable of generating a large amount of heat over the widest surface area possible. This requirement must also be harmonized with the practicality of measuring the heat flux over the entire face of the thermoelectric element.

One of the largest and most powerful thermoelectric devices that is currently available as an off-the-shelf component is the TEC2H-62-62-43775-CS (Eureca, GmbH) and was purchased for testing. This element has dimensions of $62 \mathrm{~mm} \times 62 \mathrm{~mm}$, and is capable of producing $437 \mathrm{~W}$ of cooling power at a maximum temperature difference of $80 \mathrm{~K}$. The maximum operating temperature for this device $120{ }^{\circ} \mathrm{C}$, which falls within the desired operating limits to ensure that testing does not damage the surface of the test specimen.

A corresponding off-the-shelf heat flux sensor with embedded T-Type thermocouple was found through Sequoia Technology, Ltd. This SHF6060T sensor has almost identical dimensions $(60 \mathrm{~mm} \times 60 \mathrm{~mm})$ to the desired high powered thermoelectric device, leaving only $1 \mathrm{~mm}$ of non-contact distance around the perimeter.

\subsection{Heat Sink Research}

Research was performed to evaluate the performance of different heat sinks in removing waste heat from the backside of the thermoelectric module (Figure 5.3). A variety of approaches were explored, including: i) A traditional air-cooled heat sink comprising of 
Chapter 5 - Development of a Property Measurement Device

fins coupled with an electric fan; ii) a large aluminum chunk with inherently high

conductivity and thermal capacity to act as a semi-infinite heat reservoir; iii) a passive

heat-pipe system; and, iv) a water-cooled system that includes a fan-cooled radiator. 
Chapter 5 - Development of a Property Measurement Device
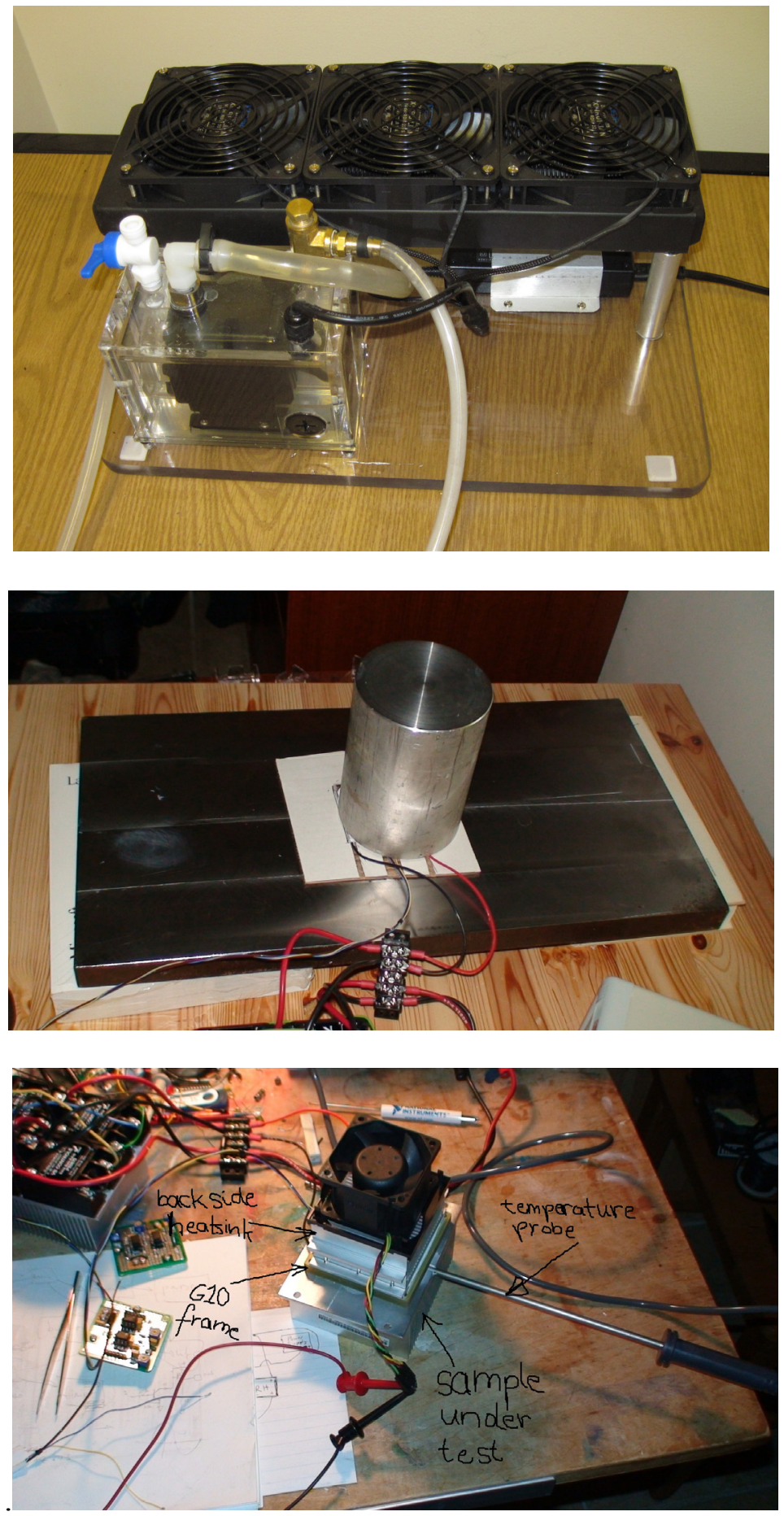

Figure 5.3 - Evaluation of various heat sink approaches 
Comparison of the benefits and drawbacks of all of the approaches are listed in Table 5.1. The outcome of this research revealed that an actively cooled heat sink is necessary to remove large amounts of extra heat generated by the thermoelectric module's inherently inefficient operation.

Table 5.1 - Heat Sink Evaluation

\begin{tabular}{|l|l|l|}
\hline Heat Sink Technique & Pro & Con \\
\hline Fins and fan & $\begin{array}{l}\text { Simple and commonly used } \\
\text { heat sink }\end{array}$ & Limited cooling power \\
chunk & $\begin{array}{l}\text { High heat capacity and } \\
\text { thermal conductivity allow } \\
\text { for the transfer and storage } \\
\text { of large amount of heat }\end{array}$ & $\begin{array}{l}\text { Thermoelectric module's } \\
\text { inherently poor } \\
\text { rise in overall } \\
\text { temperature of heat sink } \\
\text { over time }\end{array}$ \\
\hline Heat pipe and fan & $\begin{array}{l}\text { Passive device relies on } \\
\text { phase change to extract } \\
\text { large amount of heat }\end{array}$ & $\begin{array}{l}\text { Horizontal orientation } \\
\text { requirement suitable for } \\
\text { laboratory testing } \\
\text { purposes but impractical } \\
\text { for real-world testing }\end{array}$ \\
\hline Liquid cooling & $\begin{array}{l}\text { Capable of removing } \\
\text { adequate amount of Joule } \\
\text { heating generated by } \\
\text { thermoelectric module }\end{array}$ & $\begin{array}{l}\text { More complicated setup } \\
\text { and operation }\end{array}$ \\
\hline
\end{tabular}


Chapter 5 - Development of a Property Measurement Device

\subsection{Device-Armor Thermal Coupling Techniques}

Research was performed to evaluate various non-destructive methods for temporarily attaching the heater/sensor component to armored vehicles for testing purposes. The adherence technique must be able to support any position corresponding to the orientation of the armor as installed on a military vehicle (e.g. vertical, horizontal and diagonal positions). Although pressure sensitive adhesives are available, these typically require chemical/abrasive cleanup that is likely to cause some damage to the vehicle in some way or another. Superglue is commonly employed by NDE technicians when attaching probes with small surface areas. Damage to a surface is relatively minor due to the small probe size and is touched up with paint, if necessary. However, such adhesive-based coupling techniques are not acceptable for use in armor applications since damage to surface paint can compromise survivability in the field.

Suction cups provide an ideal solution since they are minimally invasive, and easy to apply and remove. These can be categorized into three classes: 1) Simple; 2) Actively controlled; and 3) Hand-pumped suction cups. Simple suction cups are not realistically feasible since they do not provide adequate resistance to loading. However, actively controlled vacuum suction cups (typically using an air compressor) are often used when dealing with extremely rough and/or porous surfaces. For sufficiently smooth surfaces, hand-pumped suction cups can be employed. 
An example of a hand-pumped suction cup is given in Figure 5.4. These are easy to operate and can carry substantial loads. An indicator on the pump actuator also warns the user of when loss of suction is imminent. Initial testing of a large surface area handpumped suction cup on various armored vehicle surfaces showed that these are capable of adhering to a surface for a long period of time (e.g. for at least 10 to 15 minutes) while maintaining a large load (greater than 100 lbs.).

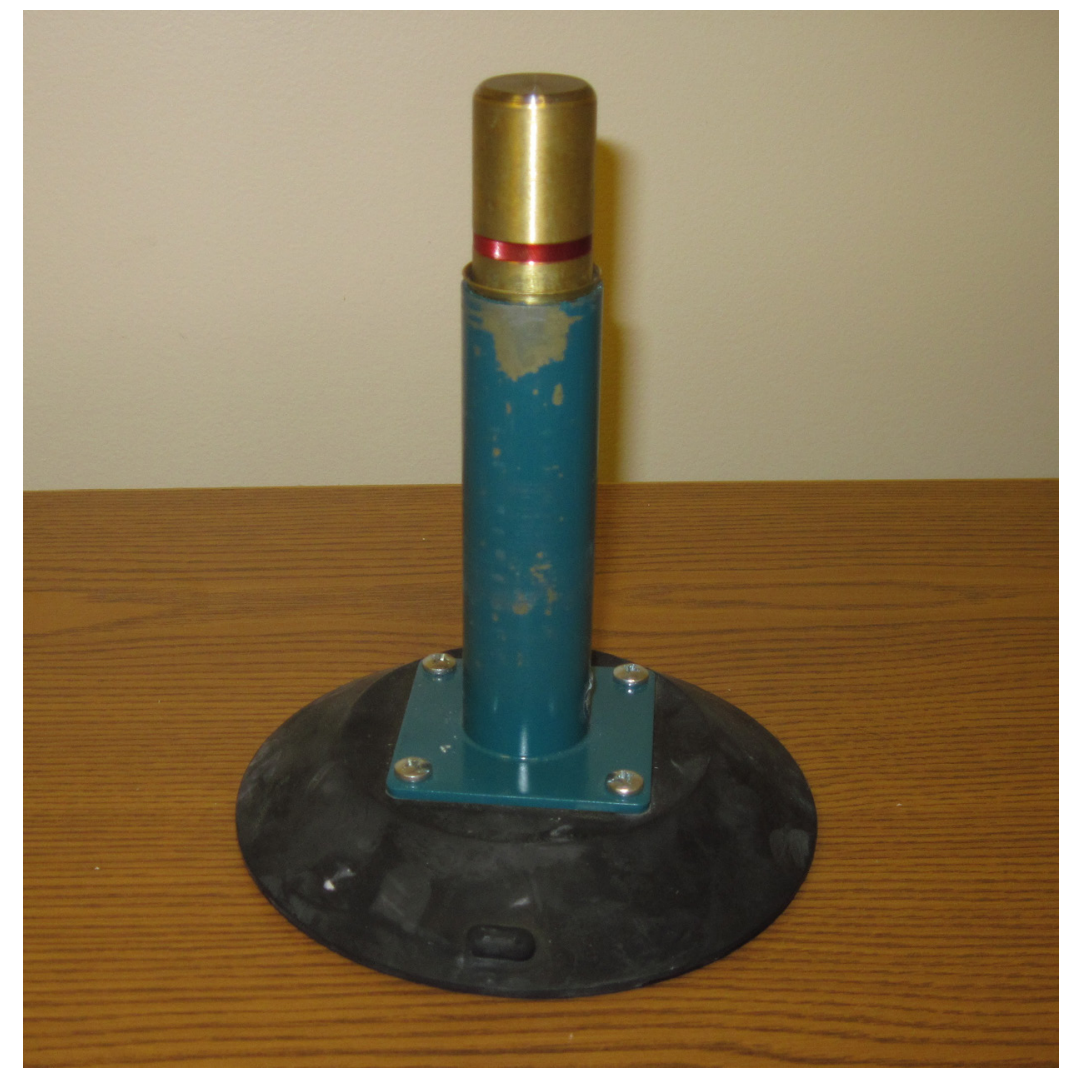

Figure 5.4 - Example of a hand-pumped suction cup 
Chapter 5 - Development of a Property Measurement Device

\subsection{Thermal Interface Material}

Optimal determination of thermal properties requires knowledge of the temperature and heat flux response within the infinitesimal surface of the test specimen. Since this cannot be accomplished from both a practical, as well as non-destructive testing perspective, it is therefore necessary to perform the measurements as close to the surface as possible, while minimizing the thermal contact resistance at the interface between the sensor and the specimen. Additionally, maximizing the heat transfer from the thermoelectric device to the test sample will also maximize the test sample response, subsequently increasing the signal to noise ratio.

A wide variety of thermal interface materials are available on the market to minimize the contact resistance between components. The intent of a thermal interface (e.g. a grease, paste, tape or pad) is to fill the microscopic air gaps inherently present due to surface roughness with a high conductivity material. Because these materials are commonly used in the high-powered computing industry to improve the conduction between electronic chips and heat sinks, a large variety of thermal interface materials are available on the open market (Figure 5.5). 
Chapter 5 - Development of a Property Measurement Device

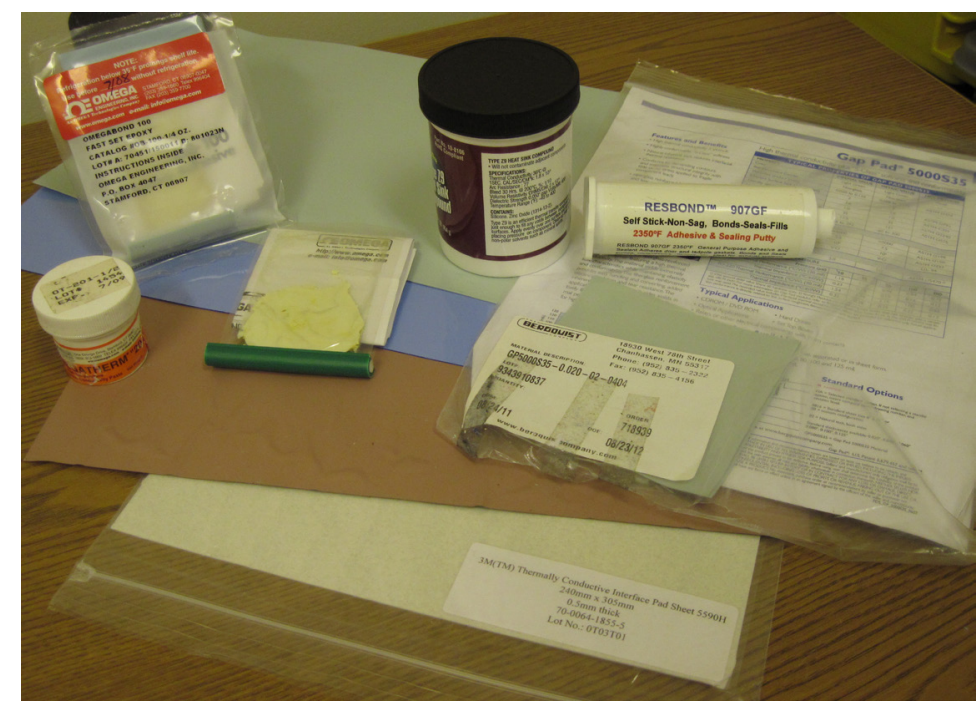

Figure 5.5 - Examples of various thermal interface materials

A review of currently available interface materials was performed based on information obtained from manufacturers' websites to provide an assessment in the differences in price, temperature range and thermal conductivity. The results of this study are shown in Table 5.2.

Table 5.2 - Thermal interface material performance

\begin{tabular}{|c|c|c|c|c|}
\hline Thermal Interface Material & Price & Temp Cycles & Features & Conductivity \\
\hline Antec 77063 Silver Thermal Compound & $\$ 10.62$ & $-60 \mathrm{C}$ to $170 \mathrm{C}$ & Made with $99 \%$ pure micronized silver & \\
\hline Coollaboratory & $\$ 24.97$ & & $100 \%$ metal & \\
\hline Evercool & & $-50^{\circ} \mathrm{C} \sim 150^{\circ} \mathrm{C}$ & & $3.8 \mathrm{~W} / \mathrm{mK}$ \\
\hline GC Electronics $10-8109$ & $\$ 8.43$ & -40 to $400^{\circ} \mathrm{F}$ & Zinc Oxide & $1.8 \times 10-3$ \\
\hline Jetart Heatpaste & $\$ 5.70$ & & CK4800 is Nano Diamond & $>8.5 \mathrm{~W} / \mathrm{m} . \mathrm{C}$ \\
\hline Kingwin Super White Freeze & $\$ 3.49$ & $-50 C-150 C$ & & $3.8 \mathrm{~W} / \mathrm{mK}$ \\
\hline $3 \mathrm{M}^{\mathrm{TM}} 8815$ Thermally Conductive AdhesiveTape & $\$ 2.95$ & & Filled Acrylic Polymer & $(\mathrm{W} / \mathrm{m}-\mathrm{K}) 0.6$ \\
\hline Masscool & $\$ 4.49$ & & & $7.5 \mathrm{~W} / \mathrm{m}-\mathrm{K}$ \\
\hline Zaward Super Thermal Grease & & $-50 \mathrm{C} \sim 170 \mathrm{C}$ & Silicone / Metal Oxide & $\sim 6 \mathrm{~W} / \mathrm{m} \mathrm{k}$ \\
\hline Zerotherm & $\$ 9.99$ & & Tape & $3.1 \mathrm{~W} / \mathrm{mK}$ \\
\hline Akasa Thermal Adhesive Tape & $\$ 4.50$ & & Tape & $0.9 \mathrm{~W} / \mathrm{m} .{ }^{\circ} \mathrm{C}$ \\
\hline
\end{tabular}


Chapter 5 - Development of a Property Measurement Device Although thermal grease appears to be the most effective interface material from a heat transfer perspective, a number of problems are associated with it from a material testing perspective. One of the major issues with grease is the uncertainty in the characterization of its thermal resistance when applied in a practical setting, which is compounded by the fact that uneven thermal grease application can further reduce its effectiveness.

Additionally, thermal grease can be cumbersome to apply and even more difficult to remove from a test surface. The most common non-destructive removal method involves multiple applications of commercial thermal grease remover and cotton swabbing; isopropyl alcohol can also be used with similar performance. Abrasive cleaners such as off-the-shelf "Goo-gone" can also be used; however, these have the potential of damaging the surface coating, which is not desirable for military vehicles from a vulnerability perspective.

Thermal interface pads appear to provide a much better fit for the proposed NDE-based thermal property measurement device than thermal grease. Although the contact resistance is greater with pads than with thermal grease, there is less uncertainty associated with the thermal conductivity and thickness of the pad since these are listed in the manufacturer's specification data sheet, providing less potential for user error and measurement inconsistency. 


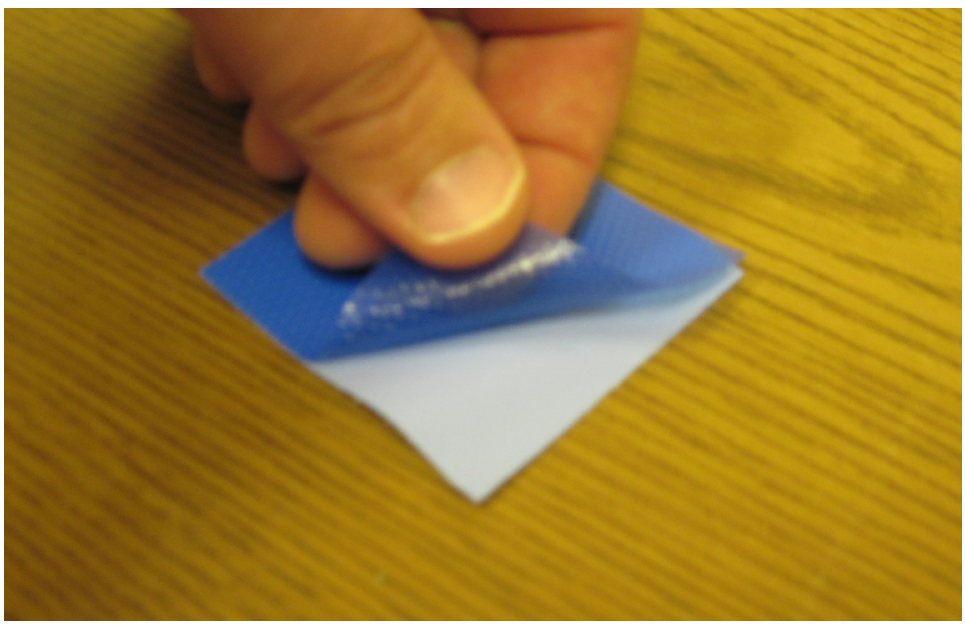

Figure 5.6 - Example of a peel-and-stick thermal interface pad

Thermal pads are relatively inexpensive, can be cut to the exact size of the heat flux sensor, are easy to apply (being available with peel-and-stick surfaces), do not require any clean-up (thereby minimizing any potential damage to a painted surface), and have the potential of being used more than once, that is, from specimen to specimen. Finally, the thermal pad can be explicitly modeled in the MuSES thermal model using the established R-value of the pad.

\subsection{Structural Design and Fabrication}

A structural component was designed and constructed to combine and fasten together the heater/sensor module with the suction cups and liquid-cooling system. This component was fabricated from aluminum in order to reduce weight and also provide strength. The design allows for the suction cup distance to be adjusted to properly position the 

heater/sensor module onto measurement surfaces that may consist of protruding elements (Figure 5.7).

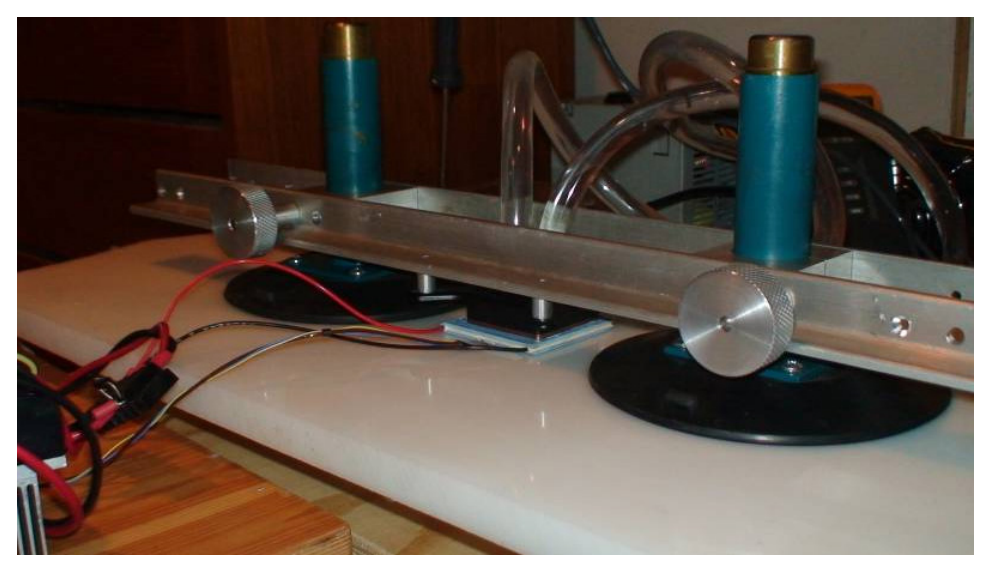

Figure 5.7 - Structural component fabricated by Motolla, LLC

\subsection{Device Calibration}

Before material testing could be performed, it was necessary to convert the raw heat flux sensor and T-Type thermocouple signals obtained from the device to valid heat fluxes and temperatures in proper units (i.e $\mathrm{W} / \mathrm{m}^{2}$ and ${ }^{\circ} \mathrm{C}$ ) so that the measurement data can be processed by the property determination code.

A special cold junction compensation (CJC) procedure was developed to work with the custom electronics of the device to account for the gain and offset error associated with the signal conditioning system that is positioned between the cold junction and the microprocessor (Figure 5.8). The voltage at the cold junction is fed through a seven pole 
analog filter, op-amp, ADC, and digital filter before it is processed and converted to a temperature signal via a multi-coefficient polynomial equation.

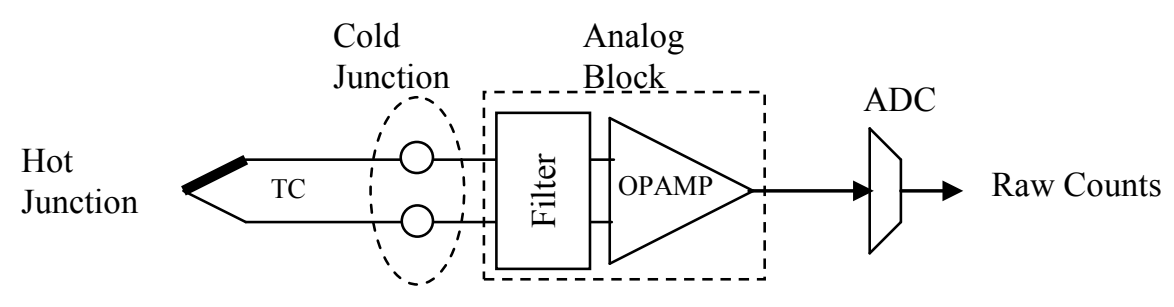

Figure 5.8 - Temperature measurement diagram showing cold junction compensation

Rather than assessing the individual voltage drops across each circuit component between the microprocessor and the cold junction, a correlation was obtained to provide a direct conversion of the reported $\mathrm{ADC}$ counts to the analog voltage required by the temperature equation. This was accomplished by successively changing the input voltage at the hot junction using the thermoelectric module until a steady signal was obtained from the TType thermocouple, and then simultaneously measuring the voltage response across the cold junction input terminals and keeping track of the ADC counts reported by the microprocessor (Figure 5.9). 


\section{ADC Counts to Thermocouple Voltage Correlation}

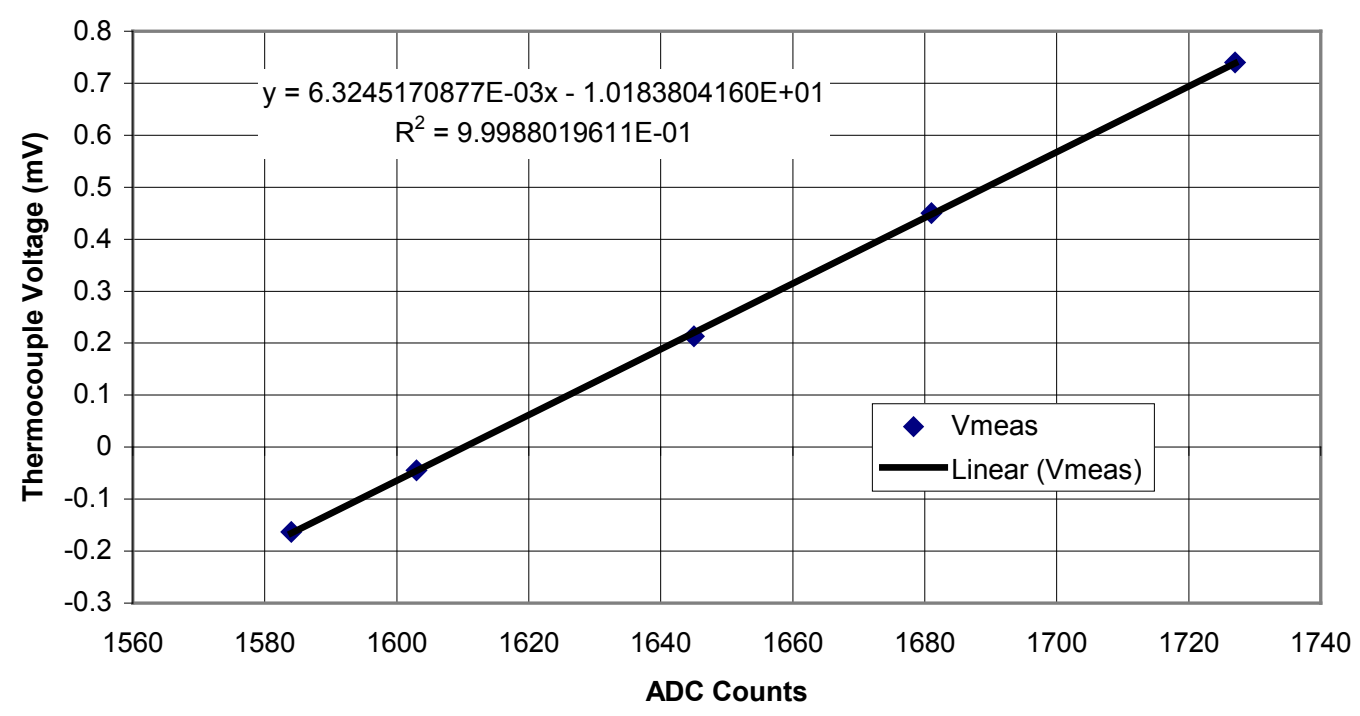

Figure 5.9- Direct correlation between ADC counts and T-Type thermocouple voltage

The T-Type thermocouple itself was calibrated by correlating its reported temperature with expected values obtained from an Omega ${ }^{\circledR}$ TrueRMS Supermeter $^{\mathrm{TM}}$ outfitted with a K-Type thermocouple. The gain and offset error obtained from this procedure were subsequently applied to the temperature predicted by the polynomial equation as a last step prior to reporting the temperature to the user (Figure 5.10). 
Thermocouple Calibration

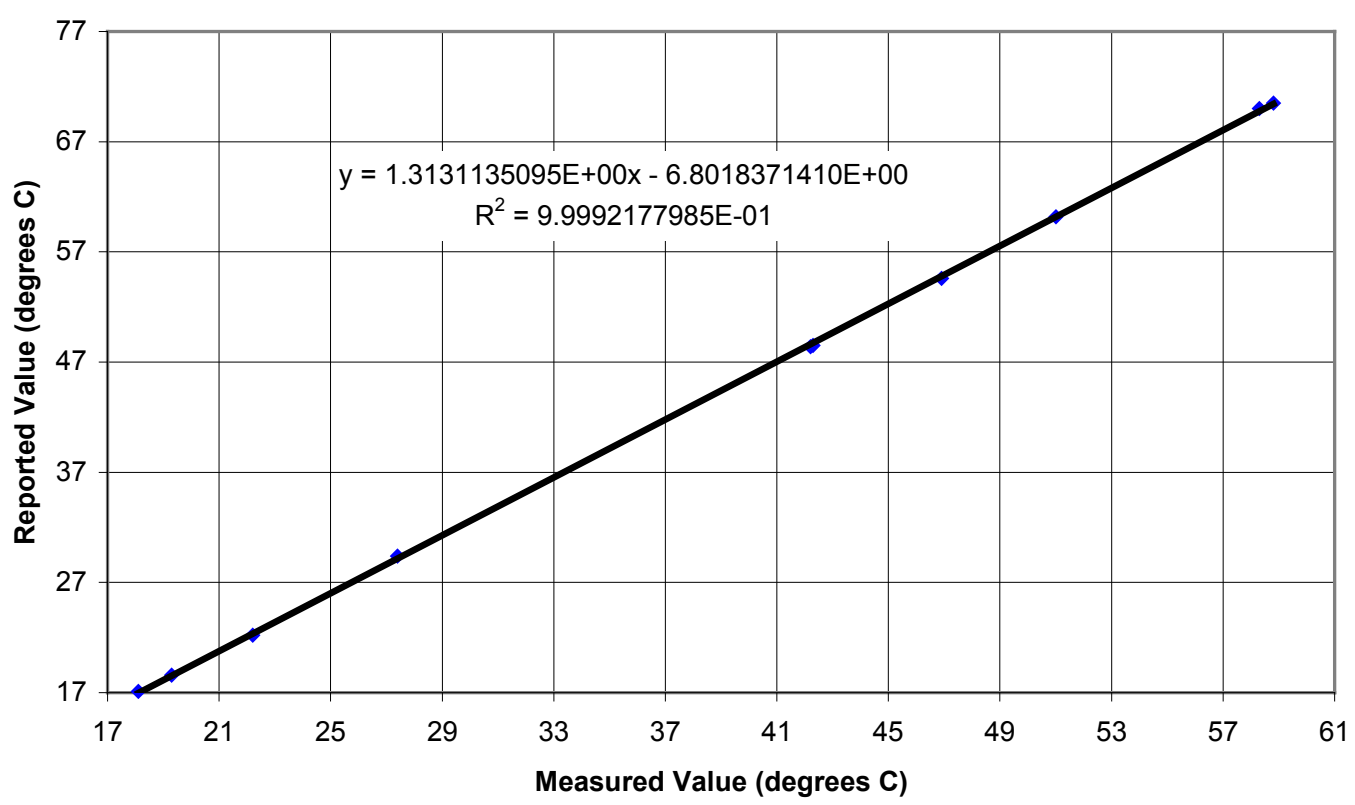

Figure 5.10- Correction for gain and offset error of the embedded T-Type thermocouple

The standard T-Type thermocouple voltage-temperature polynomial equation was developed under conditions involving a cold junction kept at a constant $0{ }^{\circ} \mathrm{C}$; however, in practical situations the cold junction temperature changes according to shifts in ambient operating temperature. The input voltage must therefore be corrected by subtracting the temperature-dependent voltage generated at the cold junction. Given the relatively narrow range of typical ambient temperatures, a thermistor can be employed to measure the temperature at the cold junction, which can be subsequently correlated to voltage by a logarithmic function (Figure 5.11). This voltage at the cold junction can then be dynamically measured and used as the reference voltage that must be subtracted from the voltage supplied to the polynomial equation. 


\section{Thermistor Calibration}

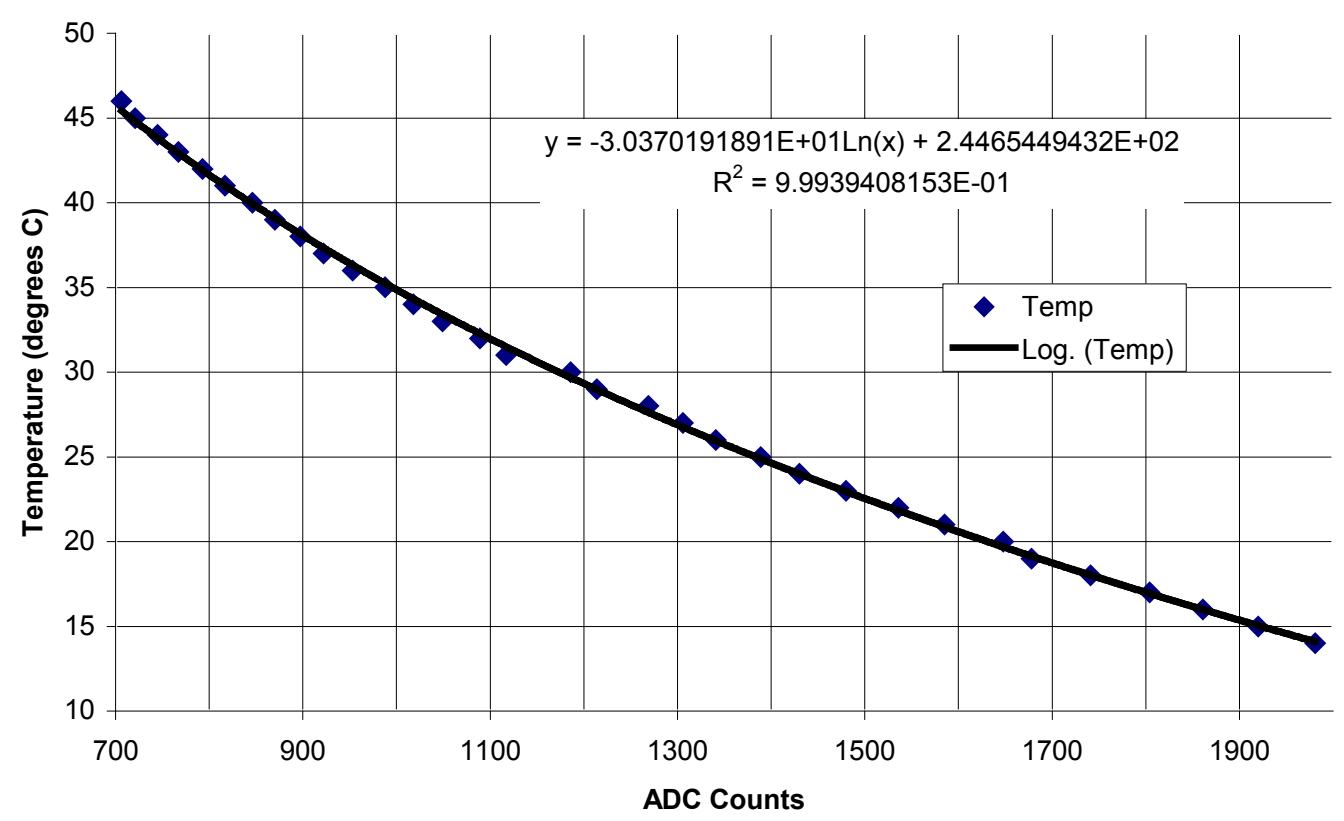

Figure 5.11- Thermistor temperature correlation to voltage-based ADC counts

Finally, a correlation between ADC counts and heat flux voltage was obtained for the heat flux sensor using a similar procedure as for that of the T-Type thermocouple (Figure 5.12). 
Heat Flux Sensor Conversion from ADC Counts to Voltage

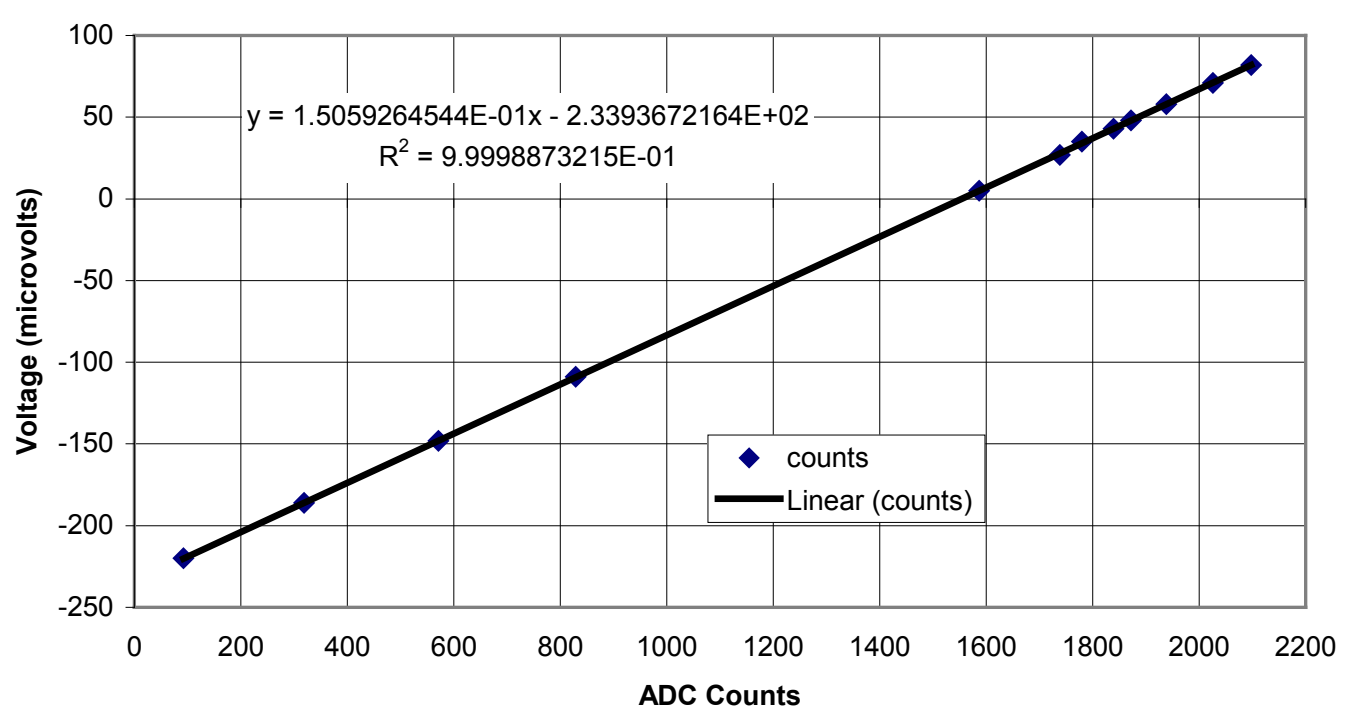

Figure 5.12- ADC Counts to voltage correlation for the heat flux sensor

The heat flux voltage is linearly proportional to the heat transferred across the component, and unlike thermocouples, can be used directly without any cold junction compensation. The proportionality constant is measured by the manufacturer and supplied with the component (i.e. $28.2 \mu \mathrm{V} / \mathrm{W} / \mathrm{m}^{2}$ ) prior to shipment.

\subsection{Property Determination Software}

A GUI-based software application was developed to measure, filter, and analyze the signals so that the material properties can be obtained from a set of test data. The underlying principle behind the code design is to provide users with a user-friendly 

interface to guide them through the entire measurement and property determination process within a single software application (Figure 5.13).
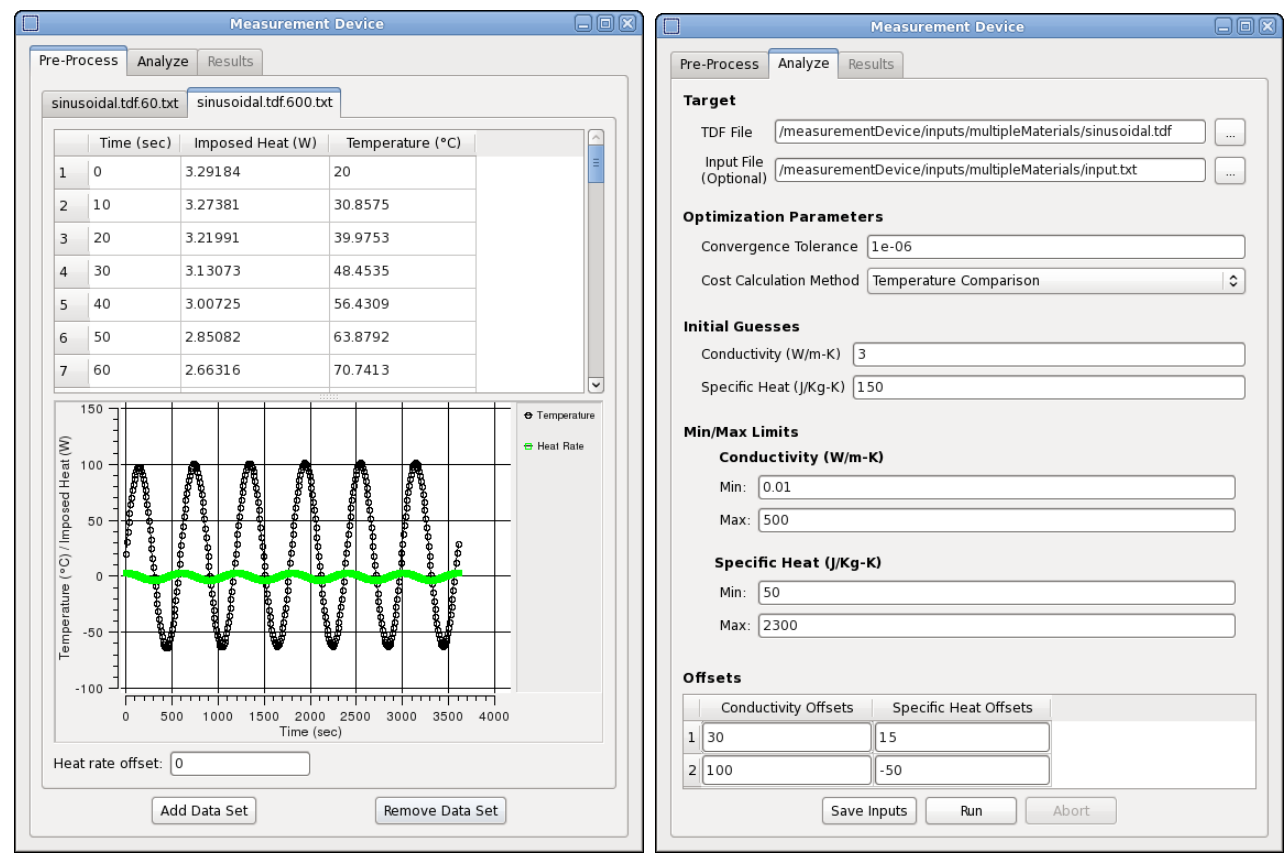

Figure 5.13- Property determination code graphical user interface

Graphing tools are provided to allow for visualization of the measurement data through every step in the process. Heat flux and/or temperature data can be visualized during data acquisition, filtering, pre-processing, and analysis.

\subsection{Laboratory Testing}

After final calibration was complete, testing of the prototype device was performed by sinusoidally heating and measuring the heat flux and temperature response on different specimens at the Motolla, LLC testing laboratory. Various materials of differing thicknesses were tested using 3,6, and 9 minute periods. The following specimens were 
tested: a 1'x1.5'x1/2" aluminum sheet (Figure 5.14); a 1'x2'x1" steel sheet (Figure 5.15); and a 1'x2'x3/4" HDPE (high density polyethylene) sheet (Figure 5.16).

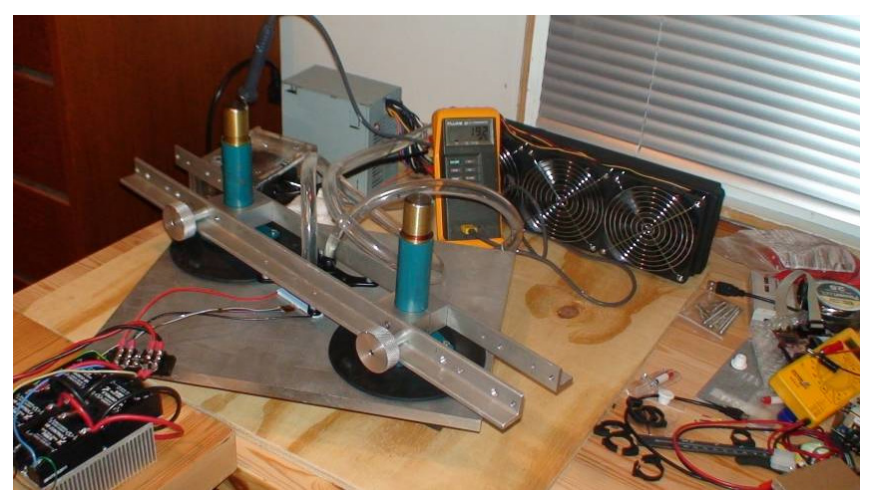

Figure 5.14 - Testing of a homogenous aluminum sheet (1'x1.5'x1/2")

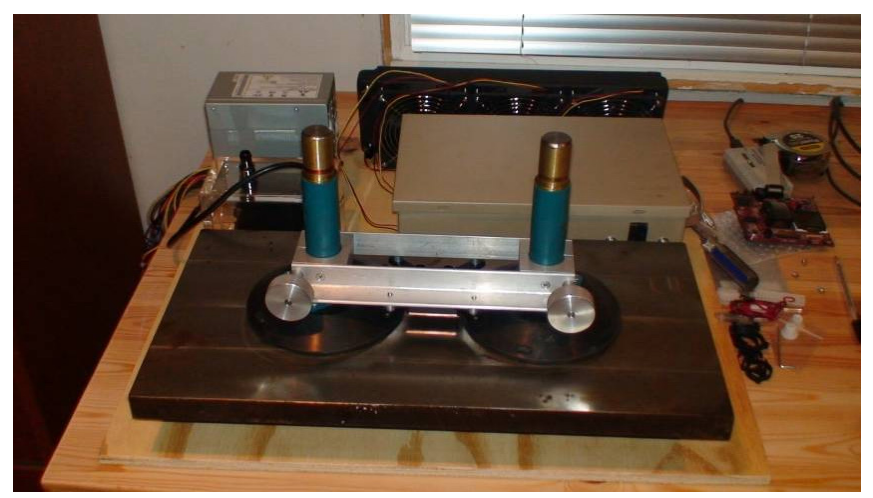

Figure 5.15 - Testing of a homogenous steel sheet (1'x2'x1")

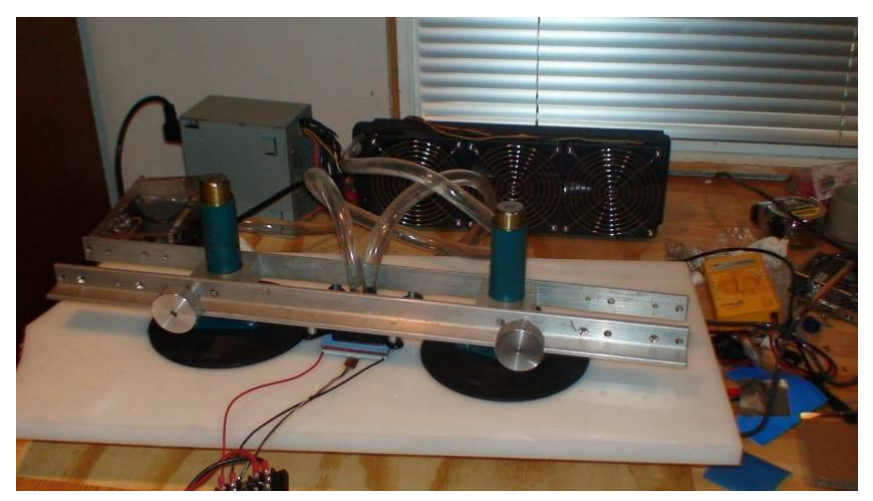

Figure 5.16 - Testing of a homogenous HDPE sheet (1'x2'x3/4") 


\subsection{Preliminary Results and Analysis}

A MuSES thermal model was constructed to replicate the experimental setup and the material properties were obtained through the built-in optimization process contained within the property determination code. The heat loss due to air convection was modeled using the built-in natural convection algorithm within MuSES using an air temperature estimate obtained from measurement of the initial water temperature of the cooling system being piped through the air-cooled radiator. The model dimensions were consistent with the dimensions of the material specimens. The heat transfer to the suction cups was not modeled since it was considered negligible. The results from these initial tests are shown in Table 5.3.

Table 5.3 - Results of preliminary homogenous material testing

\begin{tabular}{|l|c|c|c|c|}
\hline \multicolumn{1}{|c|}{ Specimen } & \multicolumn{2}{|c|}{ Conductivity (W/m-K) } & \multicolumn{2}{c|}{ Specific Heat (J/kg-K) } \\
\cline { 2 - 5 } & Measured & Expected & Measured & Expected \\
\hline Aluminum (1/2") & 160 & $167-201$ & 640 & 884 \\
\hline Steel (1") & 23 & $40-50$ & 460 & 461 \\
\hline HDPE (3/4") & 0.5 & $0.4-0.5$ & 1000 & $1550-1700$ \\
\hline
\end{tabular}

The error associated with the thermal property data obtained from the property determination code is incurred from various sources from both the modeling and measurement side. Error incurred from modeling includes mesh discretization, neglecting of temperature dependency in the optimized material properties, boundary condition error 
Chapter 5 - Development of a Property Measurement Device (uncertainty in the contact resistance between the device and the sample, convection modeling, heat loss through the suction cups), and uncertainty in characterization of the initial conditions.

The greatest boundary condition uncertainty was that of the contact resistance between the heat flux sensor and the material specimen. This resistance can be affected by the introduction of microscopic air gaps during application of the thermal pad, which can increase in size during device operation due to the thermal stresses experienced during alternating heat up and cool down cycles. The model was therefore calibrated by adjusting the thermal resistance of the gap pad to find a single value that provided the best agreement in expected material properties for all three specimens. This value was determined to be $0.002 \mathrm{~m}^{2} \mathrm{~K} / \mathrm{W}$ during the preliminary testing period. Although this resistance is greater than that listed for the pad in the manufacturer's technical sheet, it is still less than a nominal maximum corresponding to a $0.1 \mathrm{~mm}$ air gap $\left(0.00408 \mathrm{~m}^{2} \mathrm{~K} / \mathrm{W}\right)$. 
Chapter 5 - Development of a Property Measurement Device

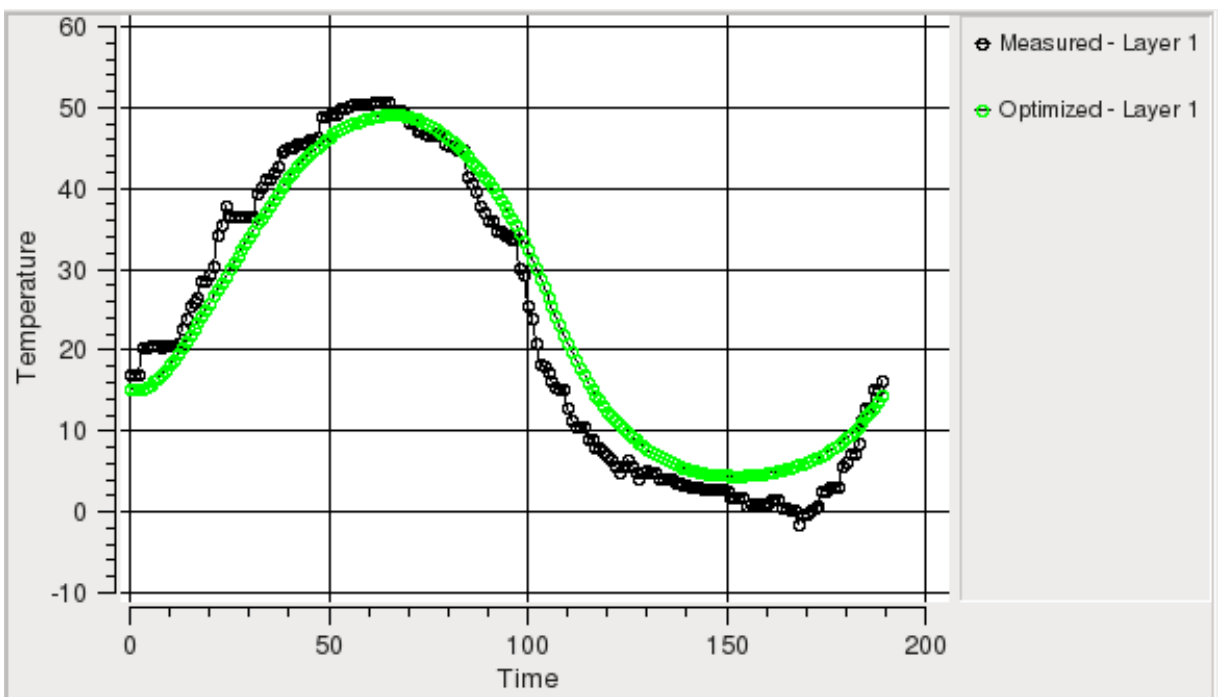

Figure 5.17- Correlation between measured temperaure data and model results

Sources of measurement derived error include both heat flux and thermocouple calibration errors as well as system noise incurred by the electronics and pulse-widthmodulation approach. Finally, since the test specimens were not measured by an independent party, there is some uncertainty in the expected results which were obtained through the literature search and/or obtained from built-in MuSES material database.

\subsection{Development of a Refined Device Prototype}

A refined device prototype was constructed by Motolla, LLC that consisted of a single component containing a built-in power supply and custom electronics (Figure 5.18). 


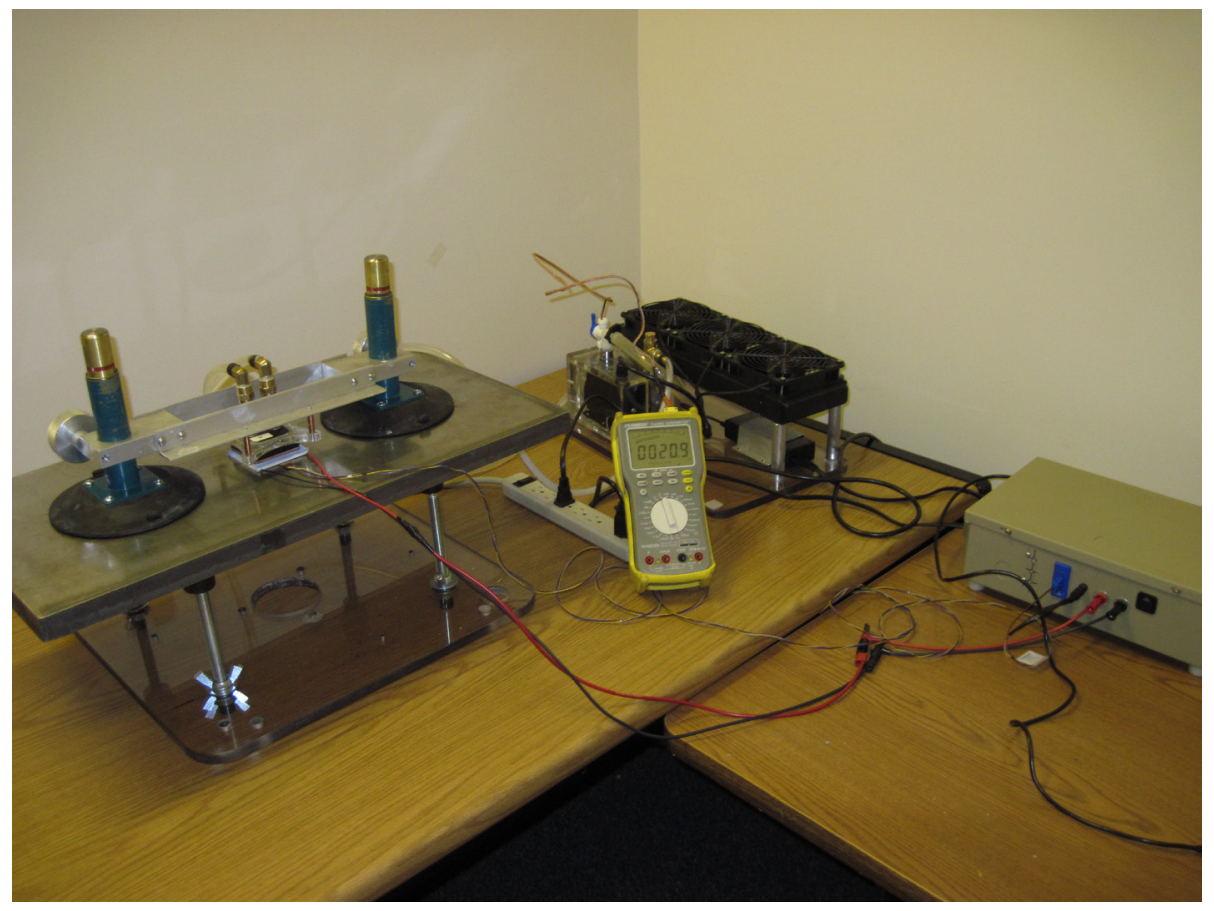

Figure 5.18 - Refined device prototype developed by Motolla, LLC

Rather than employing a traditional pulse-width-modulation approach using a constant DC voltage signal from a desktop power supply, this refined prototype used a special phase-angle-firing (PAF) method. This technique makes use of time proportioning to directly convert the $\mathrm{AC}$ power available from the grid (i.e. $60 \mathrm{~Hz}$ ) to provide sinusoidal operation of the thermoelectric module at a user defined frequency.

Use of the PAF sinusoidal control strategy provides for much more electrically quiet and gentile operation than with the traditional pulse-width-modulation approach. 
Chapter 5 - Development of a Property Measurement Device

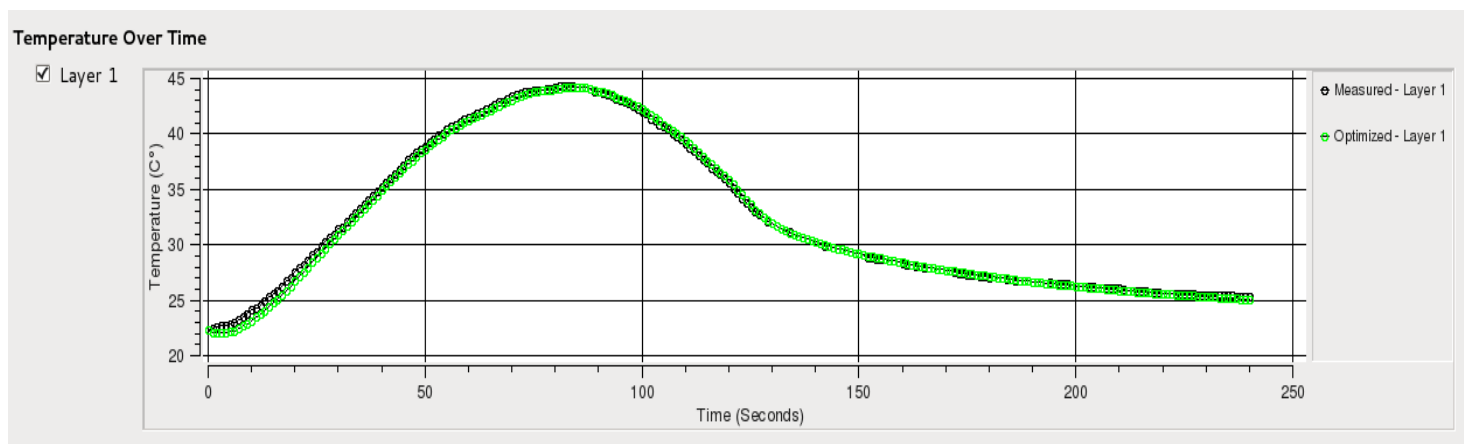

Figure 5.19 - PAF control strategy reduces electrical noise and produces cleaner measurements

The clean measurement data obtained with the refined measurement device virtually eliminates the need to pre-process the data with any filtering operations. 


\section{Chapter 6}

\section{Results and Analysis}

\subsection{Comprehensive Material Testing}

Comprehensive material testing was performed on various specimens using a wide range of driving frequencies. Although the material specimens were comprised of different thicknesses, the lengths and widths of the material specimens were carefully machined to obtain an identical surface area on the front and back faces (1'x2'; $30.48 \mathrm{~cm} \mathrm{x} 60.96 \mathrm{~cm})$; this consistency was maintained in order to facilitate the thermal modeling side of the analysis (Table 6.1).

Table 6.1 - Materials and layered stack-ups used for testing

\begin{tabular}{|l|l|l|l|l|l|l|}
\hline Thickness & $1 / 4 "$ & 1 " & $1 / 4 "$ & $3 / 4 "$ & 1 "(1/4" over $3 / 4 ")$ & 1 " (3/4" over 1/4") \\
\hline Material & Steel & Steel & Aluminum & HDPE & Steel over HDPE & HDPE over Steel \\
\hline
\end{tabular}

The range of frequencies employed was based on the capabilities of the device. For example, the maximum frequency that the device prototype was able to support (at the time of testing) was $0.03333 \mathrm{~Hz}$, while the minimum frequency was based on practical limitations, since the prototype device would periodically stop operating during tests involving long durations. At least two cycles worth of temperatures and heat fluxes were acquired for all of the specimens at a minimum frequency of $0.002083 \mathrm{~Hz}$. Testing was 
therefore performed at the following frequencies (periods): $0.03333 \mathrm{~Hz}$ (30 seconds), $0.01667 \mathrm{~Hz}$ (60 seconds), $0.01111 \mathrm{~Hz}$ (90 seconds), $0.008333 \mathrm{~Hz}$ (120 seconds), $0.004167 \mathrm{~Hz}$ (240 seconds), and $0.002083 \mathrm{~Hz}$ (480 seconds).

The heat flux and temperature results from the tests were further processed so that they could be represented in terms of the frequency response of the material specimens. The gain, expressed as the output $\left(\mathrm{T}_{\max }-\mathrm{T}_{\min }\right)$ over the input $\left(\mathrm{q} "{ }_{\max }-\mathrm{q} "{ }_{\min }\right)$, and the phase shift, measured with respect to the effective zero crossings of the input and output signals, were obtained for each experiment and shown in Figure 6.1 and Figure 6.2.

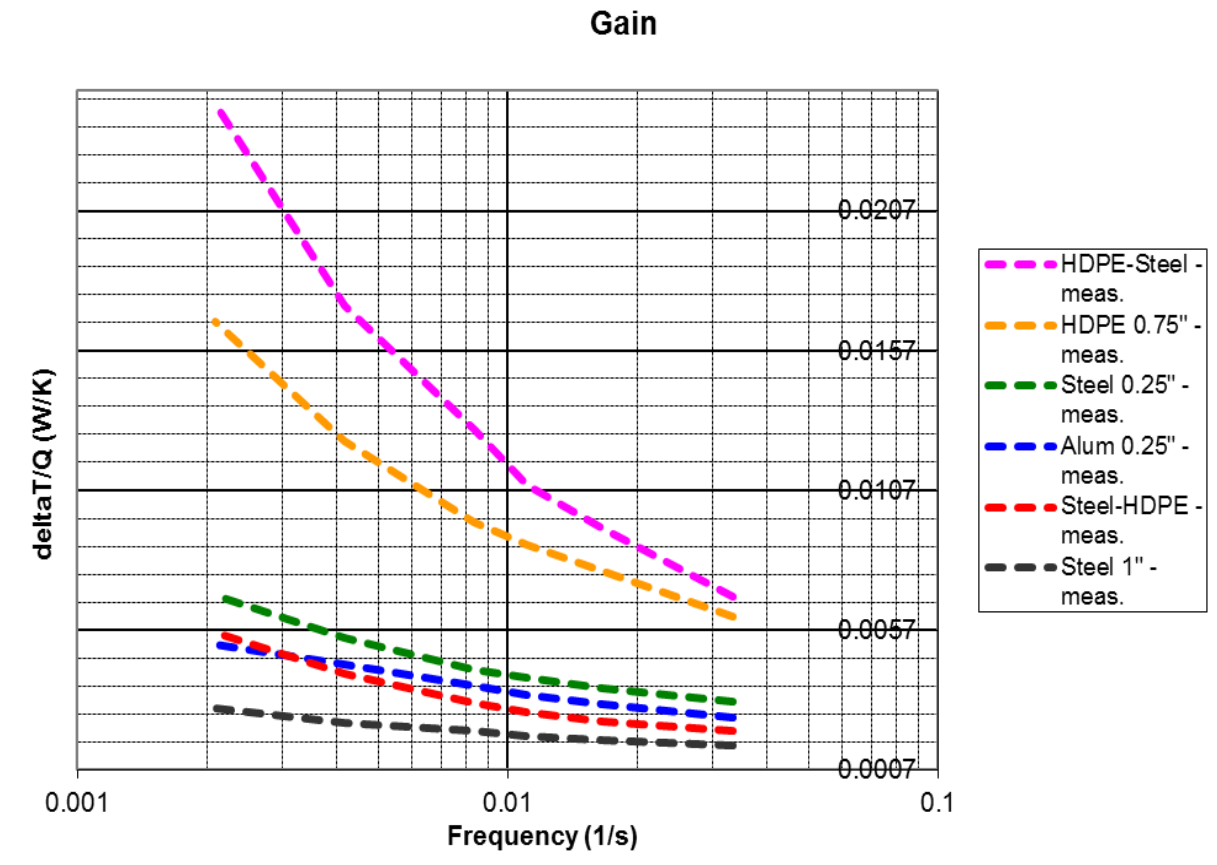

Figure 6.1 - Gain response for range of applied sinusoidal heating frequencies 


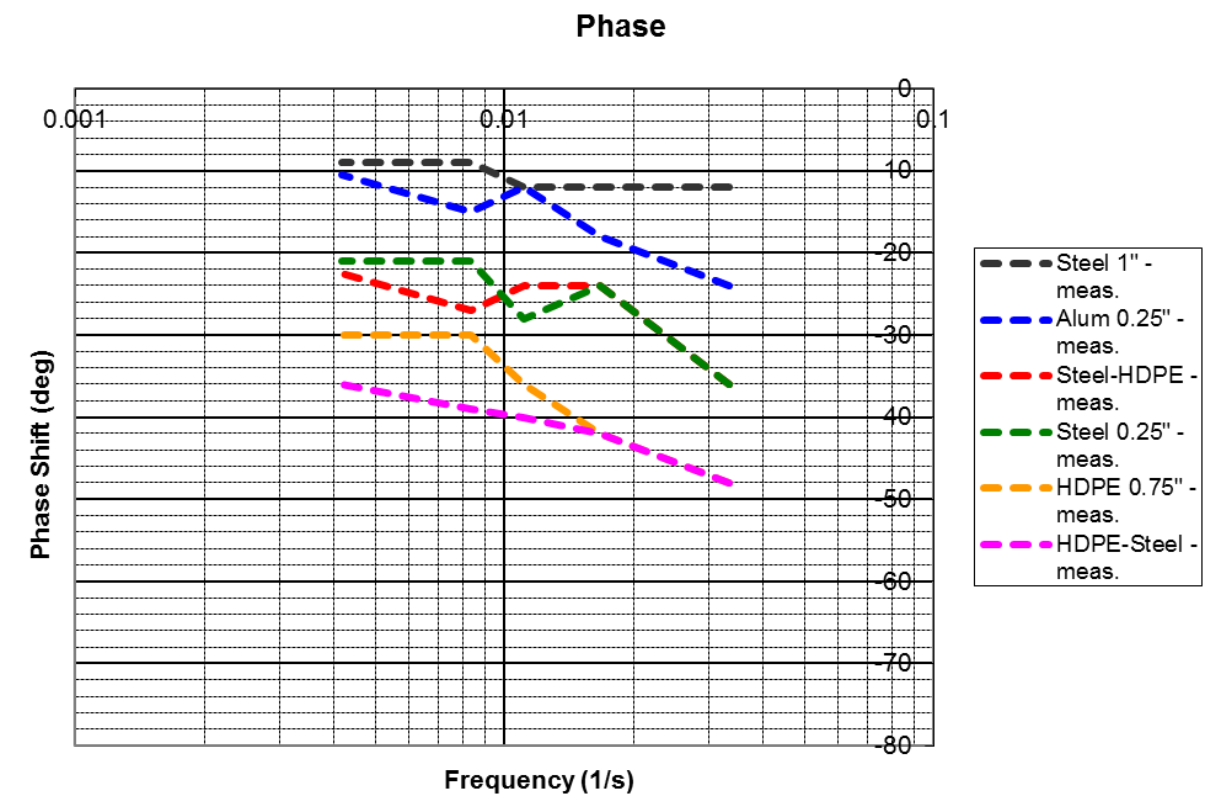

Figure 6.2 - Phase response for range of applied sinusoidal heating frequencies

Simulations were subsequently performed by supplying the measured heat fluxes from each corresponding test as the driving boundary condition for the material specimen. The material properties were approximated by employing nominal values for steel, polyethylene, and aluminum obtained from the MuSES database. A nominal gap pad resistance of $0.001 \mathrm{~m}^{2}-\mathrm{K} / \mathrm{W}$ was used for all simulations. The results of these simulations are provided in terms of their frequency response (Figure 6.3 and Figure 6.4). 
Chapter 6 - Results and Analysis

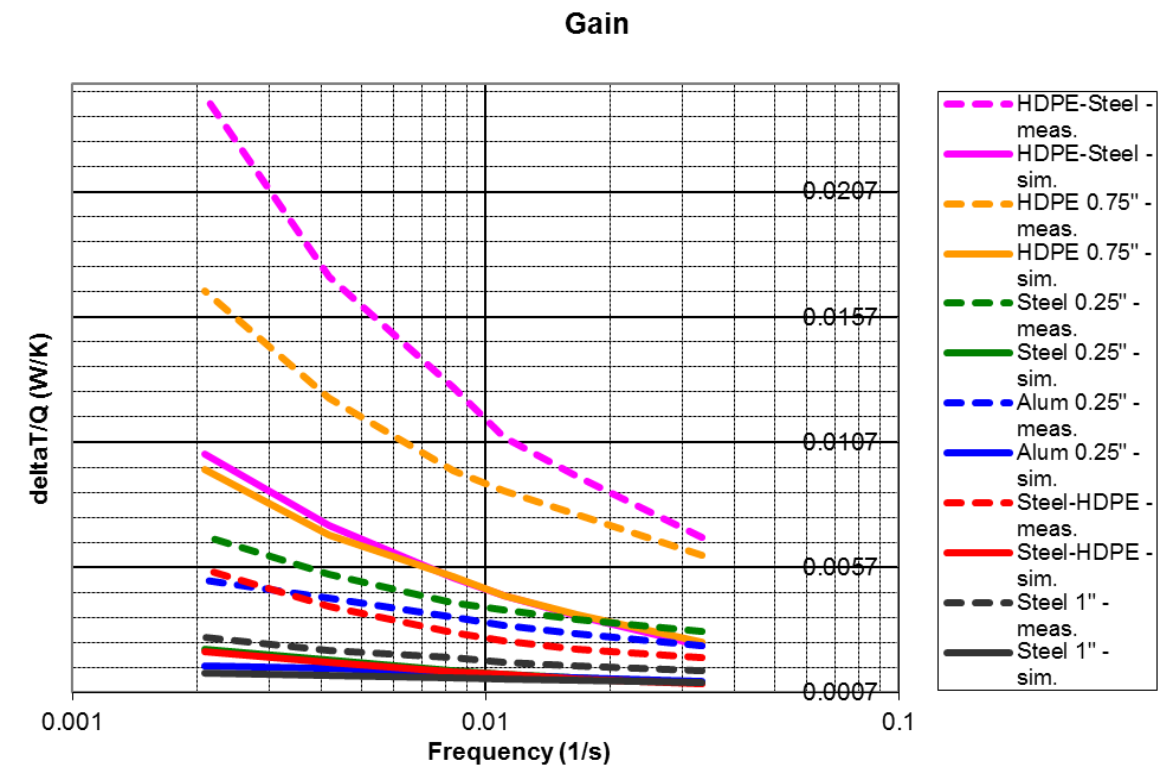

Figure 6.3 - Simulated gain response compared with measurements

\section{Phase}

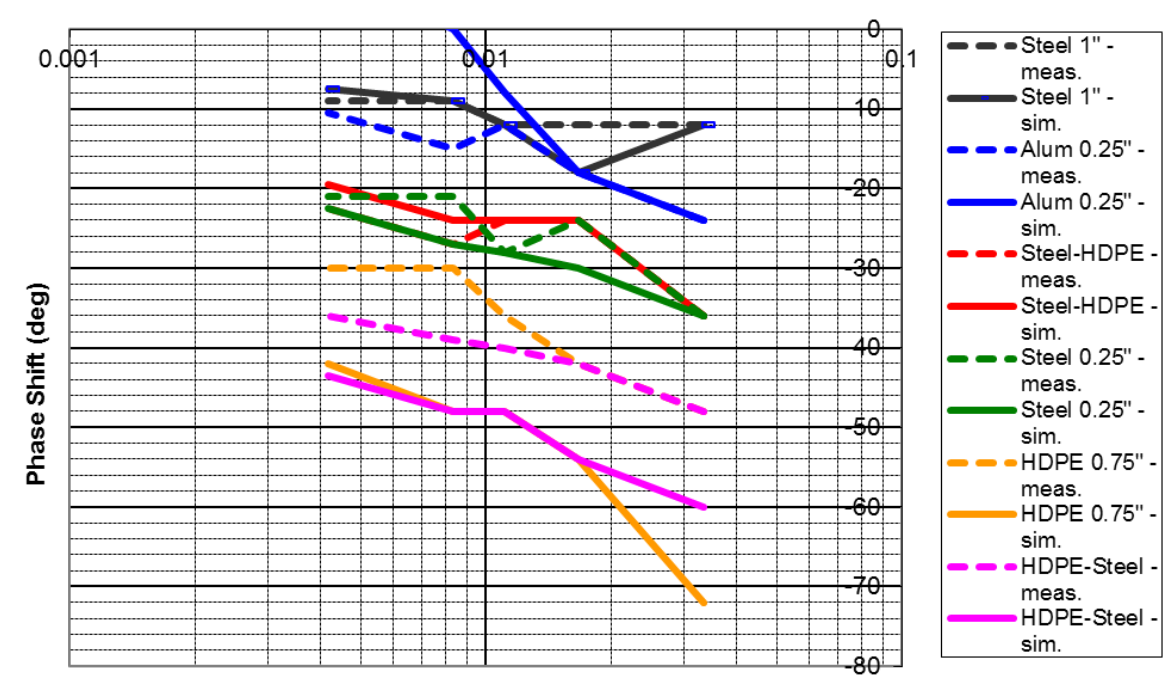

Frequency (1/s)

Figure 6.4 - Simulated phase response compared with measurements 


\subsection{Application of a Gain Error Calibration Factor}

A parametric study was performed that involved varying the thermal resistance of the thermal interface and the boundary conditions in model; however, none of these parameters significantly affected the gain in the frequency response of the simulated materials. However, it was found that the simulated frequency response correlation to the measured response could be improved by applying a consistent gain error factor to the measured heat flux that was used to drive the simulations,. For example, applying a nominal gain factor of 2.0 to the input heat flux to the simulation provided the improved correlation between the measured and simulated gain and phase frequency response, as seen in Figure 6.5 and Figure 6.6.

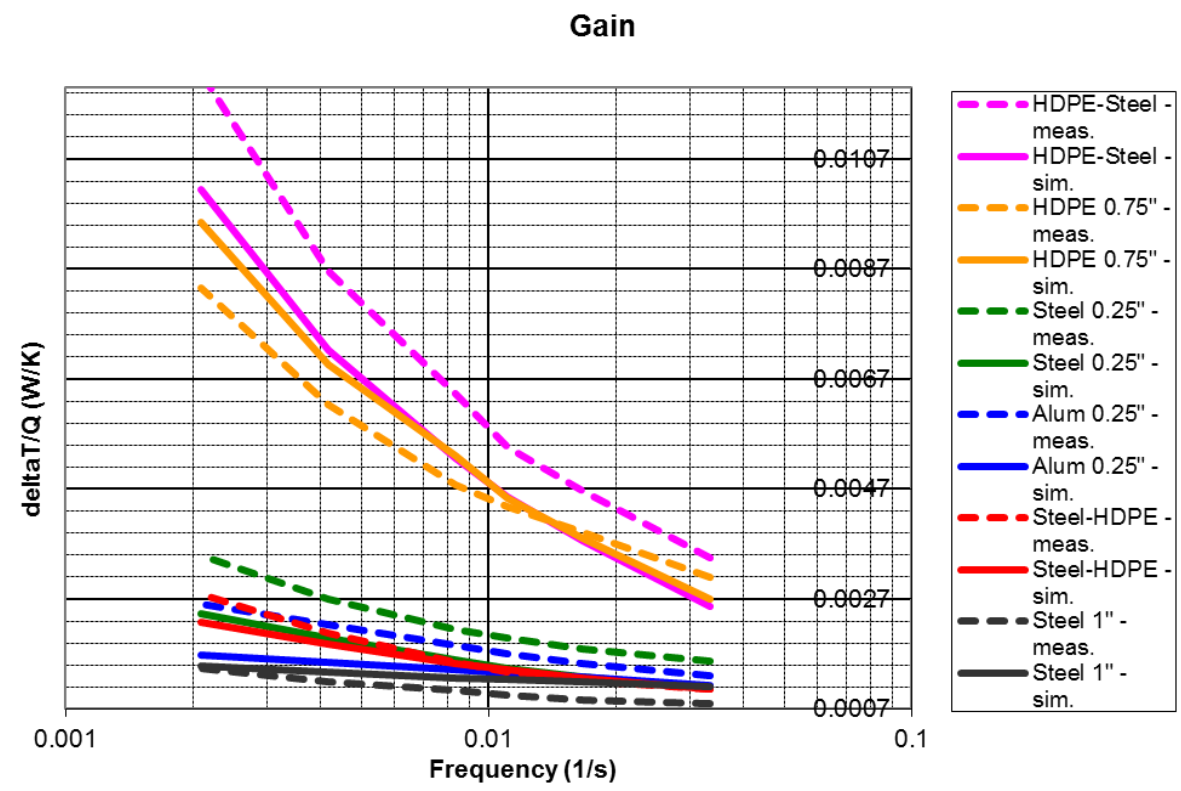

Figure 6.5 - Gain of the frequency response of simulated samples using a gain factor of 2.0 


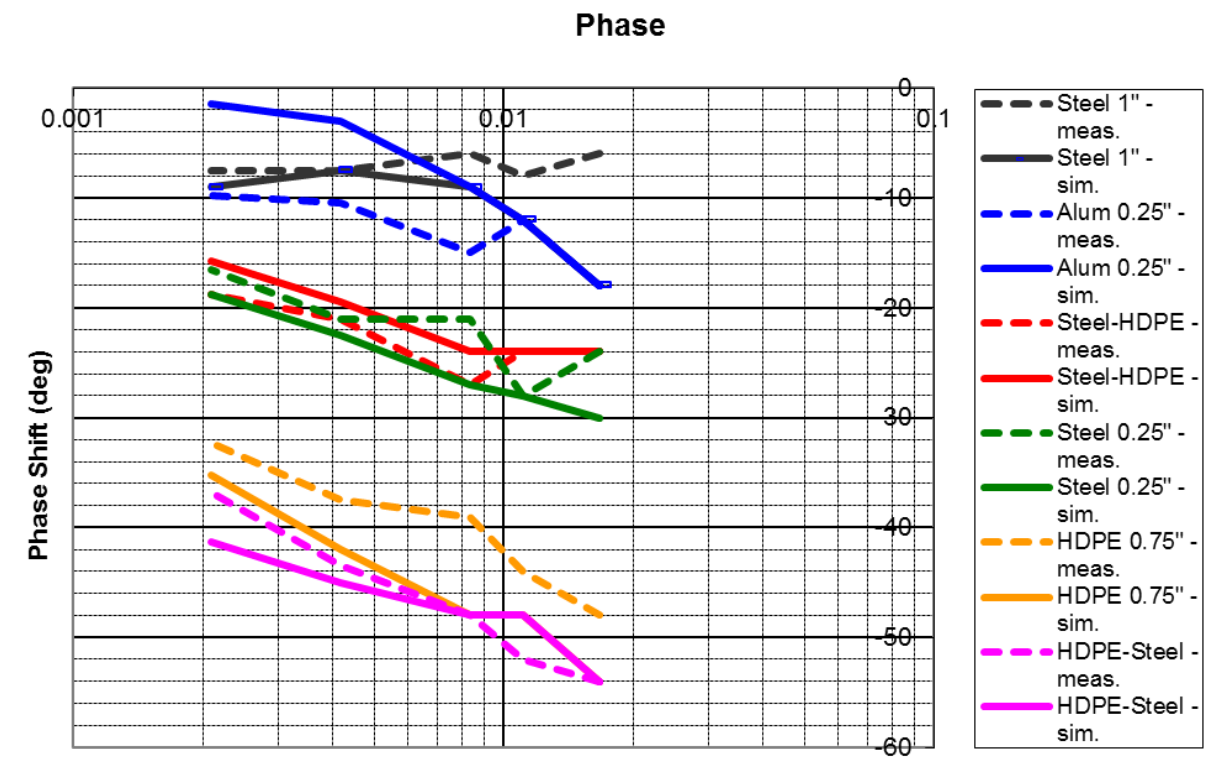

Frequency (1/s)

Figure 6.6 - Phase of the frequency response of simulated samples using a gain factor of 2.0

Additionally, the use of a gain factor provided an improved correlation between the measurements and simulations for all frequencies, considering that perfect correlation was not expected, since the properties of the samples were approximated. The slopes of the lines, along with their relative order from top to bottom within the plots, are consistent from material to material and frequency to frequency. Additionally, with respect to the HDPE and steel stack-ups, the simulations assume a perfect bond at the interface between them, while in actuality these materials were held together using thermal grease and C-clamps. 


\subsection{Heat Flux Sensor Calibration Study}

An experiment was performed to assess the validity of the heat flux sensor calibration factor provided by the manufacturer. To accomplish this, a $60 \mathrm{~mm} \times 60 \mathrm{~mm}$ calibration coupon was machined out of $1 / 2$ " HDPE so that a 1-D heat transfer problem could be formulated to quantify steady-state heat transfer through the sample, based on the measured temperatures and the thermal conductivity of HDPE (Figure 6.7, Figure 6.8).

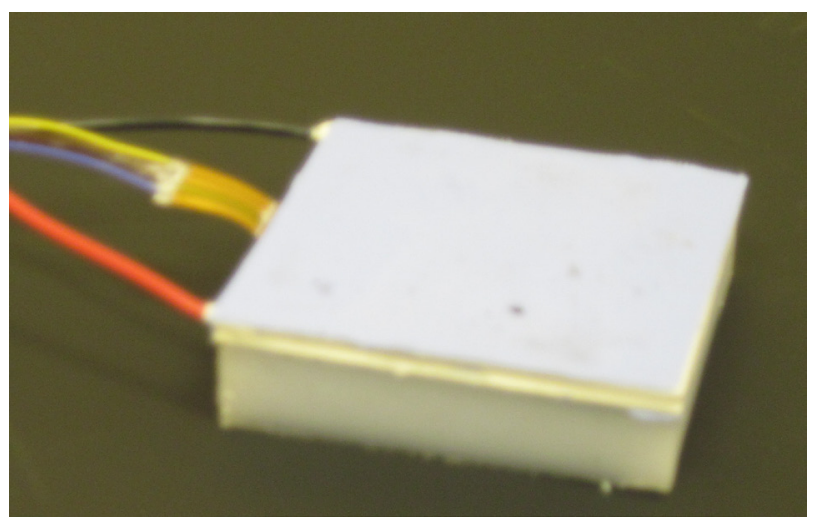

Figure 6.7 - HDPE calibration sample connected to heat flux sensor and Peltier module

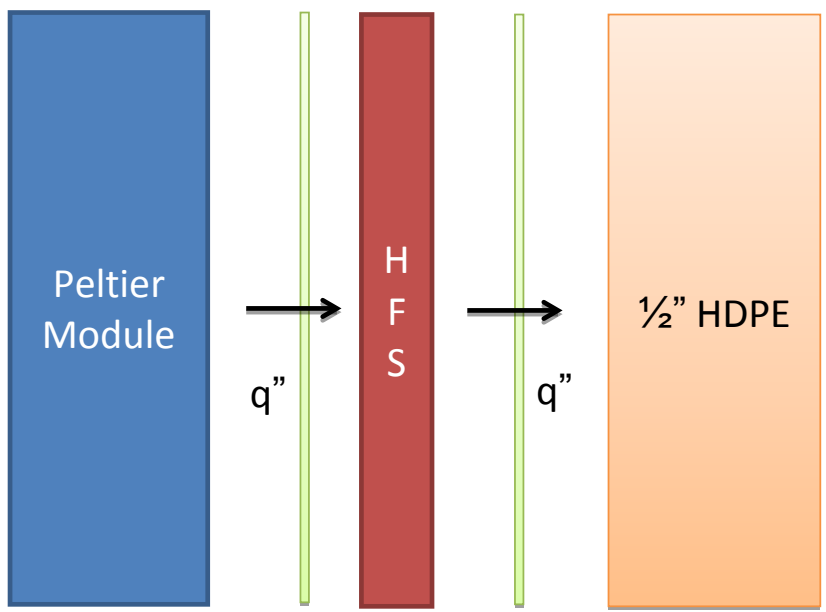

Figure 6.8 - Heat flux sensor calibration setup

The test was performed at $50 \%$ power output to the Peltier module. Once the system reached its steady-state, the measured temperatures on both faces of the HDPE coupon 
were $54.7^{\circ} \mathrm{C}$ and $49.2{ }^{\circ} \mathrm{C}$, respectively. Using a nominal conductivity of $0.43 \mathrm{~W} / \mathrm{m}-\mathrm{K}$ for the HDPE, an expected heat flux of $185.7 \mathrm{~W} / \mathrm{m}^{2}$ was calculated, which provided excellent correlation with the reported heat flux from the device, $186.6 \mathrm{~W} / \mathrm{m}^{2}$. The thermal model was also modified to conform to this calibration test, which provided results that were in agreement with the calculated heat flux under steady-state.

In order to examine the transient response of the heat flux sensor, a subsequent test was carried out to measure the transient backside temperature. For this test, the Peltier module was lowered to $40 \%$ of its maximum power to shorten the time to steady-state. Although at steady-state the use of the measured heat flux as a boundary condition in the model produced temperatures that were in agreement with the measurements, during the transient phase of the experiment the predicted temperature response was much lower than the measured values. In contrast, using the measured front side temperature as a boundary condition to the simulation resulted in a backside temperature prediction that was in agreement with the measurements. However, the predicted heat flux was almost double the measured value during the predominantly transient phase of the simulation, which decayed towards the reported measured value of heat flux as the system reached its steady-state (Figure 6.9). 


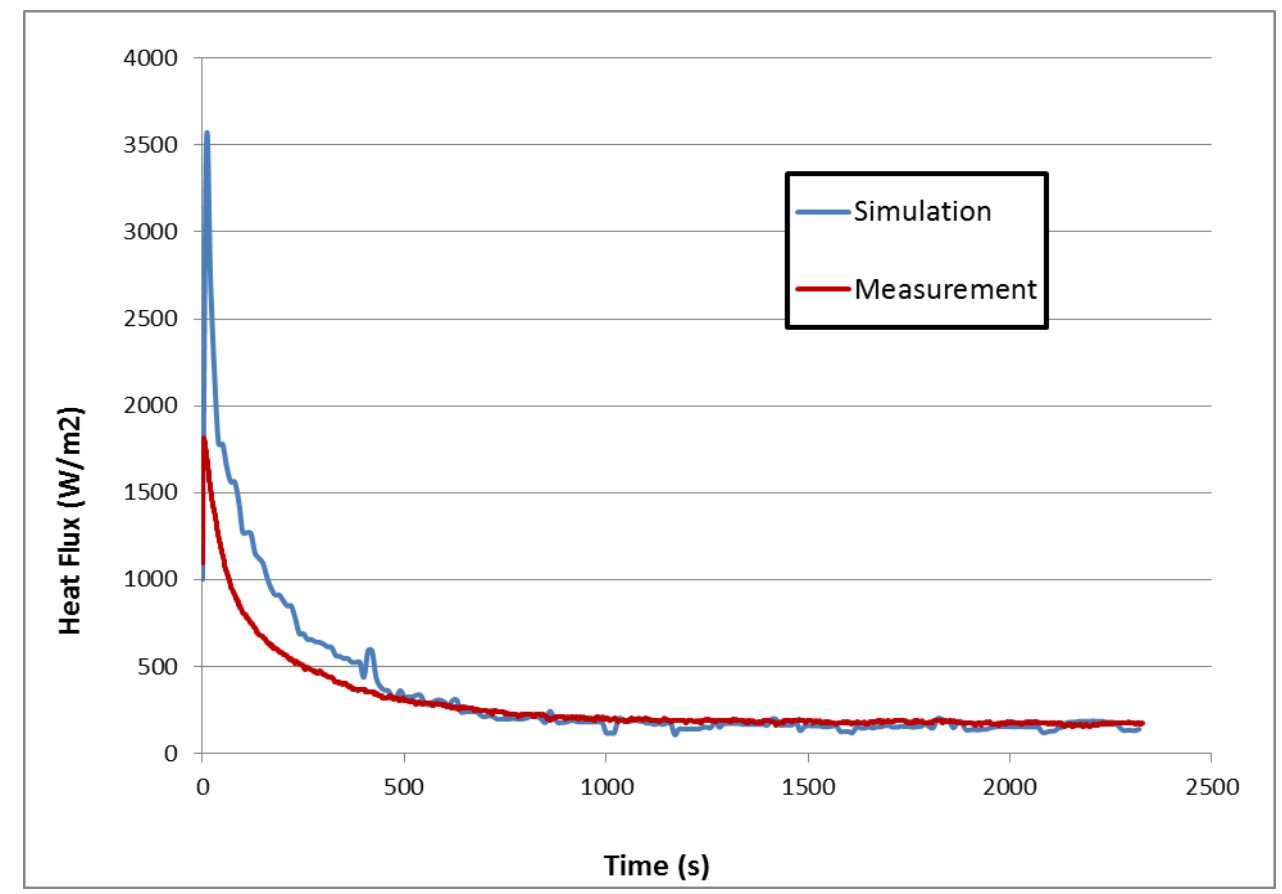

Figure 6.9 - Comparison of measured heat flux with simulated heat flux (constant power)

The model's results were compared against an analytical solution (i.e. the semi-infinite solid), to confirm the validity of the temperature predictions. The results of the analytical solution were consistent with the numerical model, which further indicated that almost double the required heat was required to provide the necessary temperature response that was measured.

The test coupon was further subjected to a sinusoidal heat input, with varying amplitudes. Application of the measured temperature as a boundary condition to the model resulted in a consistently greater heat flux prediction as compared to the measured value (i.e., by about a factor of 2). The measured and predicted results are given in Figure 6.10. 


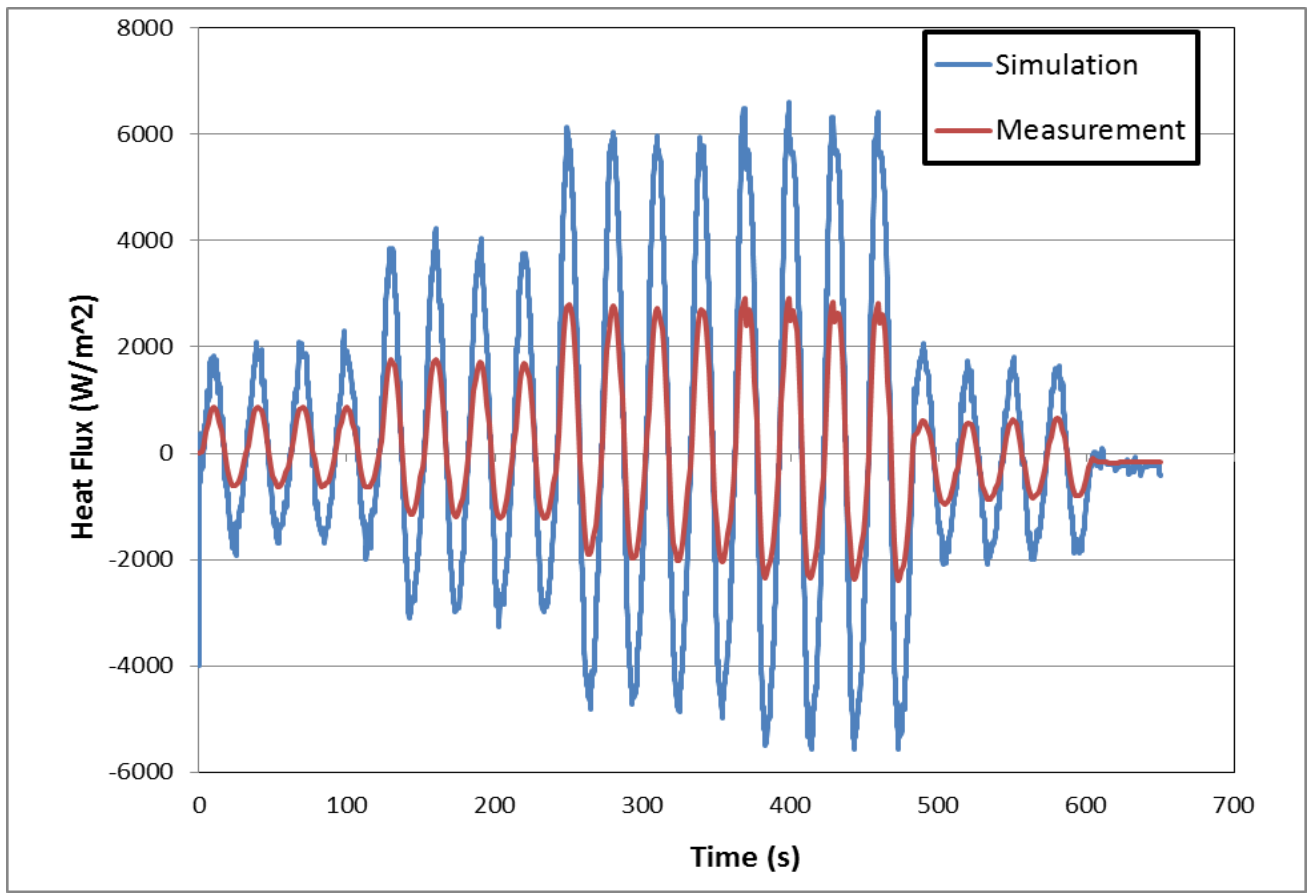

Figure 6.10 - Comparison of measured heat flux with simulated heat flux (sinusoidal power)

Based on the results of this preliminary testing, it was hypothesized that the error was introduced by the sensor's inability to properly integrate the heat flux across its face in the presence of a non-uniform temperature distribution. The source of the non-uniformity was suspected to be caused by the particular Peltier module that was being used. Thermal imagery was subsequently obtained using an IR camera pointed at an exposed Peltier module when operating at a constant power output to confirm the validity of this theory. It was found that a non-uniform temperature is indeed present during operation of this particular module, being clearly evidenced in the image provided in Figure 6.11. 


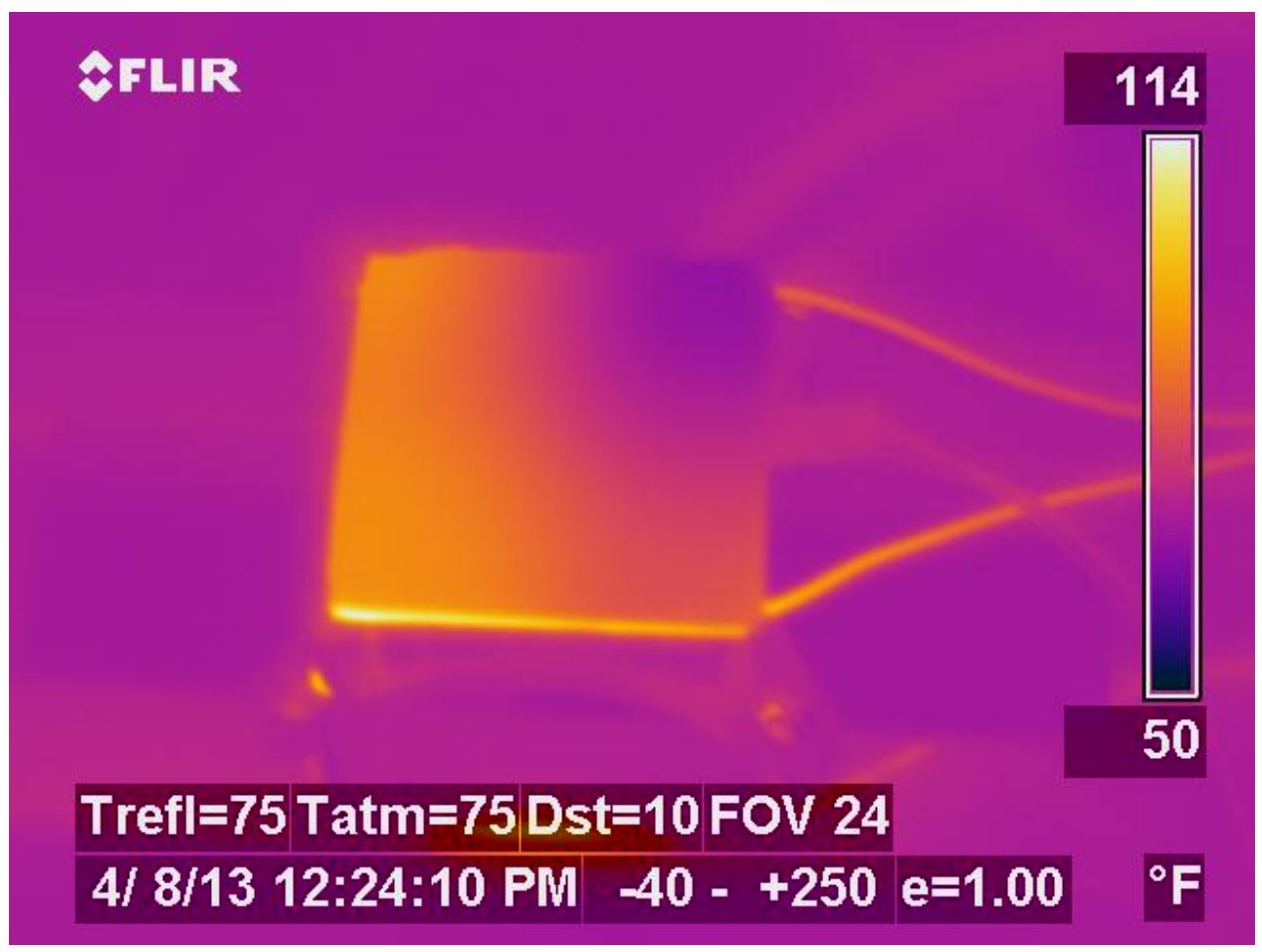

Figure 6.11 - Thermal image showing non-uniform temperature at Peltier module surface

Further testing of the heat flux sensor was therefore performed to evaluate its accuracy under non-uniform temperatures. A test was conducted in which the bottom of the heat flux sensor was held at constant temperature near $50{ }^{\circ} \mathrm{C}$ (the heat source), while the top of the sensor was exposed to two heat sinks operating at different temperatures. The primary heat sink was driven by an ice bath, while the secondary heat sink maintained ambient temperature. The secondary sink essentially maintained a temperature between that of the primary heat sink and the source. The contact area was varied in relation to the primary heat sink $(100 \%, 75 \%, 50 \%, 25 \%$, and $0 \%$ coverage $)$. Analytical solutions were computed based on the measured temperatures and a nominal contact resistance of 0.004 $\mathrm{m}^{2} \mathrm{~K} / \mathrm{W}$. The ratios of the calculated and reported (measured) heat flux for each of the 
measurements is given in Figure 6.12.

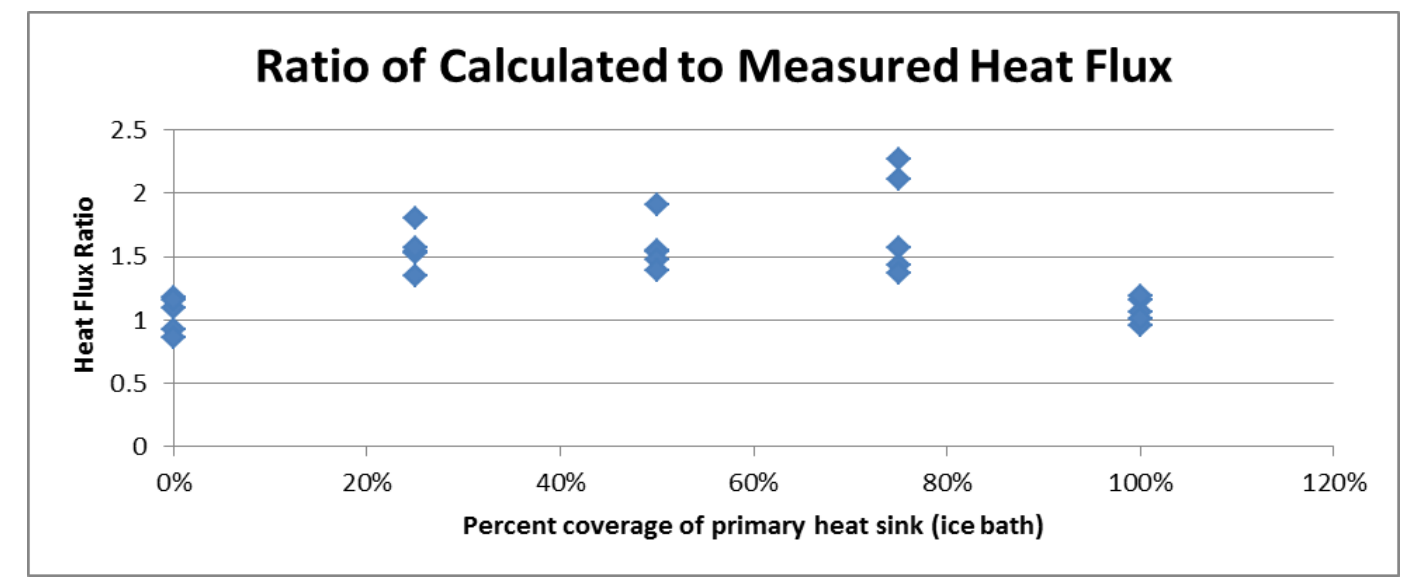

Figure 6.12 - Ratio of calculated to measured heat flux

The results of these experiments clearly show that the reported heat flux value is not equal to the calculated (expected) value when the sensor is exposed to non-uniform temperatures at one of its surfaces.

\subsection{Property Determination of Homogeneous Materials}

Despite the measurement error introduced by the non-uniform temperature at the sensor face, an attempt at property determination was undertaken to assess the quality of material properties obtainable through the use of an additional calibration gain factor that would be appropriate for sinusoidal heating. Unfortunately, exhaustive analysis failed to produce a consistent calibration gain factor for all materials and frequencies. Specifically, if the supplied gain factor was overestimated or underestimated, the optimization routine 
would diverge. In spite of this, the best compromise gain factor for the available data was found to correspond to a value of approximately 1.9. Although convergence was not always possible for all materials and frequencies, the gain factor value produced relatively good agreement with expected material properties when used in combination with a thermal interface resistance bounded by 0.0007 and $0.0016 \mathrm{~m}^{2} \mathrm{~K} / \mathrm{W}$.

A definite frequency dependence on the gain factor was discovered when processing material properties from the measured data. For example, this can be seen in the values obtained for the $3 / 4$ " HDPE specimen, provided in Table 6.2. While the properties obtained from the measurements taken with the 480 second period correlated well with nominal values $\left(\mathrm{k} \approx 0.5 \mathrm{~W} / \mathrm{m}-\mathrm{K}, \mathrm{c}_{\mathrm{p}} \approx 1500 \mathrm{~J} / \mathrm{kg} / \mathrm{K}\right)$, the accuracy of the predictions is shown to decrease with increasing frequency.

Table 6.2- Material property results obtained from $3 / 4$ " HDPE at different frequencies

\begin{tabular}{|r|r|r|r|r|r|r|}
\hline $\begin{array}{l}\text { Period } \\
(\mathbf{s})\end{array}$ & $\begin{array}{l}\boldsymbol{R}_{\text {interface }} \\
\left(\boldsymbol{m}^{2} \mathbf{-} \boldsymbol{K} / \boldsymbol{W}\right)\end{array}$ & $\begin{array}{l}\text { Gain } \\
\text { Factor }\end{array}$ & Cost & Convergence & $\begin{array}{l}\boldsymbol{k} \\
(\boldsymbol{W} / \boldsymbol{m}-\boldsymbol{K})\end{array}$ & $\begin{array}{l}\boldsymbol{c}_{\boldsymbol{p}} \\
(\boldsymbol{J} / \boldsymbol{k g}-\boldsymbol{K})\end{array}$ \\
\hline 30 & 0.0009 & 1.9 & 0.307 & $5.03 \mathrm{E}-07$ & 2.03 & 179.6 \\
\hline 60 & 0.0009 & 1.9 & 0.062 & $5.34 \mathrm{E}-07$ & 1.53 & 278.1 \\
\hline 90 & 0.0009 & 1.9 & 0.033 & $8.32 \mathrm{E}-07$ & 1.29 & 378.5 \\
\hline 120 & 0.0009 & 1.9 & 0.024 & $6.31 \mathrm{E}-07$ & 1.11 & 492.7 \\
\hline 240 & 0.0009 & 1.9 & 0.014 & $9.57 \mathrm{E}-07$ & 0.69 & 977.8 \\
\hline 480 & 0.0009 & 1.9 & 0.009 & $8.82 \mathrm{E}-07$ & 0.53 & 1356.1 \\
\hline
\end{tabular}

Without a sufficiently large gain factor to enable the predicted temperature to reach the corresponding measured amplitude, the optimizer attempts to reconcile the mismatch between the model and the measurement by decreasing the specific heat capacity. The 
potential phase lag introduced by this adjustment is subsequently counter balanced by the optimizer with an increase in the predicted conductivity.

\subsection{Multilayer Material Property Determination}

Further material testing was performed on multilayered stack-ups to evaluate the extent and accuracy of material properties that can be obtained for layers residing deeper within the test specimen. To accomplish this, the measurement data obtained from the steel over HDPE stack-up (i.e., an outer sheet of 1/4" steel over 3/4" HDPE, sandwiched together using thermal grease and traditional C-clamps) was employed, as depicted in Figure 6.13.

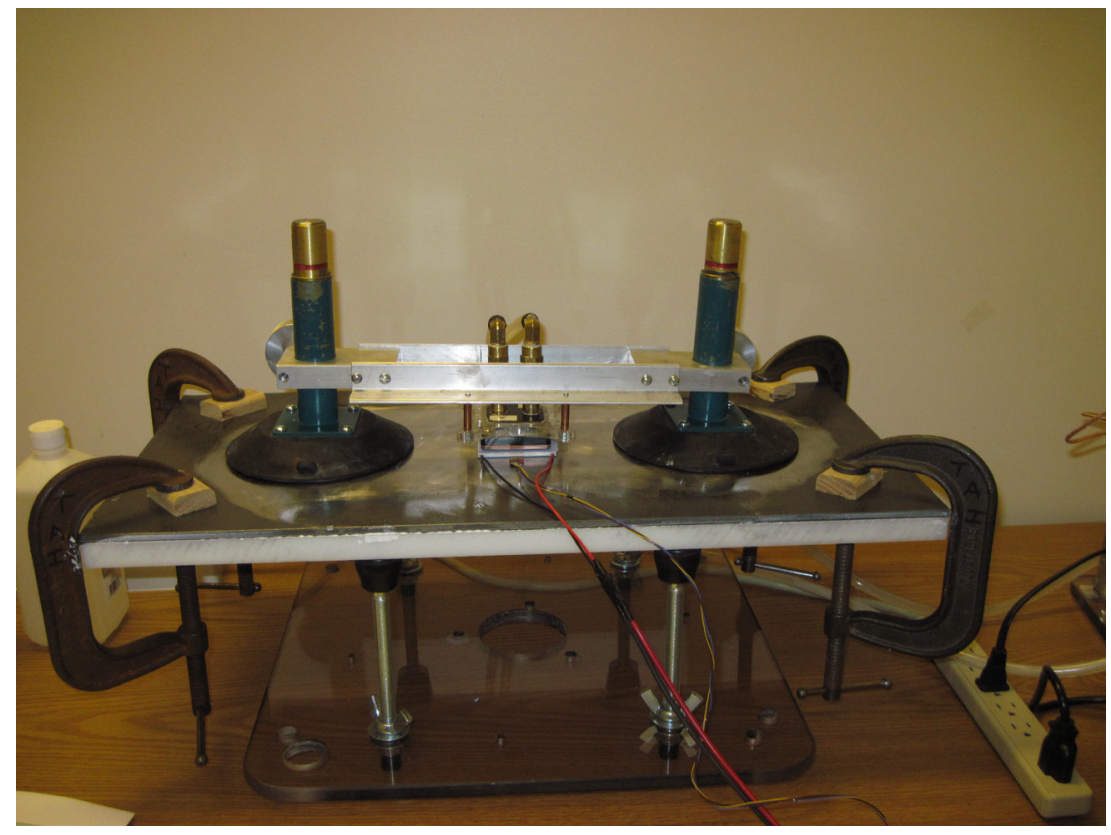

Figure 6.13 - Testing of a steel over HDPE layered stack-up 
In order to make use of a single gain factor, while keeping in mind its minor frequency dependence, it was necessary to obtain the effective material properties from applying only the three shortest periods ( 30 seconds, 60 seconds, and 90 seconds), since convergence was not found to be feasible when using all seven available data sets. The following process was therefore employed to obtain the thermal properties of the three effective layers:

1. The measurement data associated with the highest frequency was used to obtain the effective conductivity and specific heat of the first layer, by solving for its material properties by treating the entire specimen as one homogenous material.

2. The properties obtained from step one were subsequently applied to the front layer and held constant while the optimization routine attempted to find a single effective conductivity for the rest of the specimen by using measurement data obtained from the lowest frequency.

3. Finally, the properties obtained from step 2 were subsequently applied to the back layer. An additional degree of freedom is introduced by making use of the middle frequency to solve for the middle layer, while holding the material properties of both the front and back layers constant (Figure 6.14). 


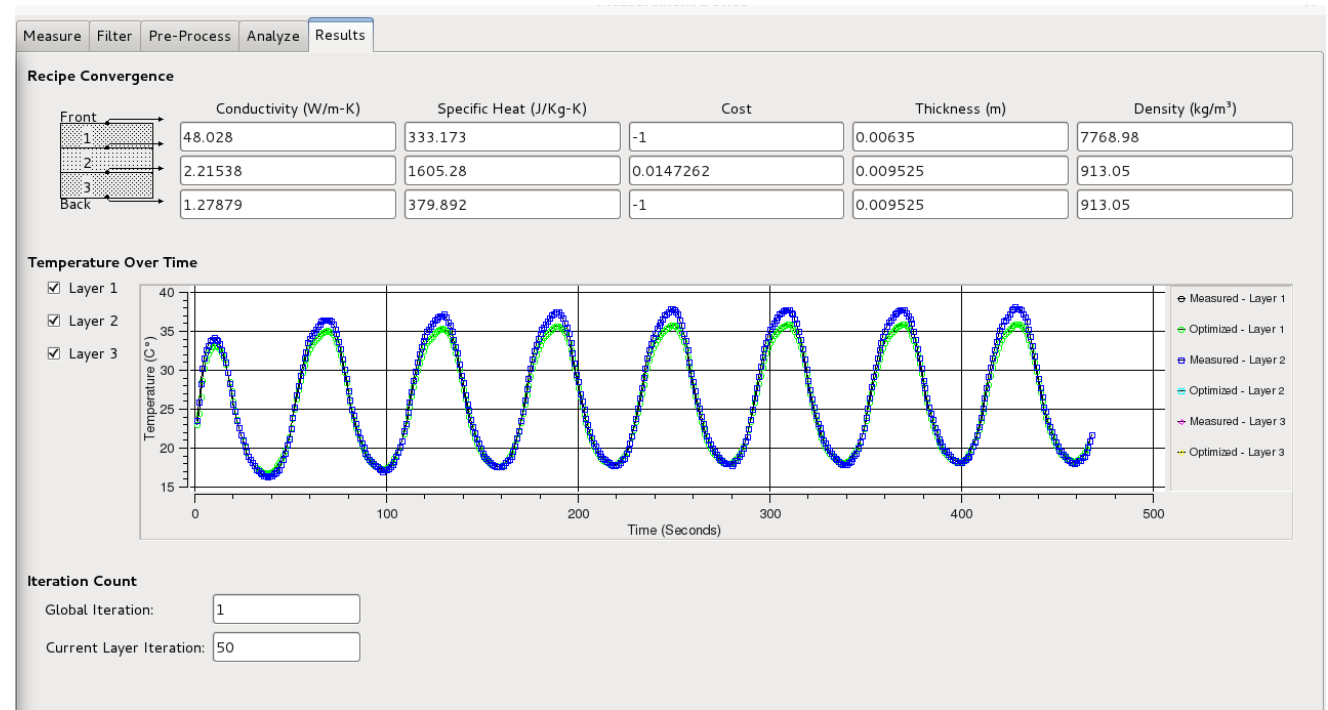

Figure 6.14 - Multilayer material thermal property determination

This iterative procedure yielded the material properties presented in Table 6.3. These results show good agreement relative to the expected nominal properties of a layered steel over HDPE stack-up; $\left(\mathrm{k} \approx 50 \mathrm{~W} / \mathrm{m}-\mathrm{K}, \mathrm{c}_{\mathrm{p}} \approx 460 \mathrm{~J} / \mathrm{kg} / \mathrm{K}\right)$ and $\left(\mathrm{k} \approx 0.5 \mathrm{~W} / \mathrm{m}-\mathrm{K}, \mathrm{c}_{\mathrm{p}} \approx\right.$ $1500 \mathrm{~J} / \mathrm{kg} / \mathrm{K}$ ), respectively. Specifically, the thermal conductivity was found to be much lower in the second and third layers than in the first layer, while the specific heat of the second layer was found to be very close to the nominal specific heat of HDPE.

Table 6.3- Results of multilayer material property determination of a steel over HDPE stack-up

\begin{tabular}{|r|l|r|r|r|r|r|r|}
\hline $\begin{array}{l}\text { Layer } \\
\#\end{array}$ & Material & $\begin{array}{l}\text { Period } \\
(\mathbf{s})\end{array}$ & $\begin{array}{l}\boldsymbol{R}_{\text {interface }} \\
\left(\boldsymbol{m}^{2}-\boldsymbol{K} / \boldsymbol{W}\right)\end{array}$ & $\begin{array}{l}\text { Gain } \\
\text { Factor }\end{array}$ & Cost & $\begin{array}{l}\boldsymbol{k} \\
(\boldsymbol{W} / \boldsymbol{m}-\boldsymbol{K})\end{array}$ & $\begin{array}{l}\boldsymbol{c}_{\boldsymbol{p}} \\
(\boldsymbol{J} / \mathbf{k g}-\boldsymbol{K})\end{array}$ \\
\hline 1 & Steel & 30 & 0.0009 & 1.9 & 0.0322 & 48.0 & 333.2 \\
\hline 2 & HDPE & 60 & 0.0009 & 1.9 & 0.0147 & 2.22 & 1605.3 \\
\hline 3 & HDPE & 90 & 0.0009 & 1.9 & 0.0141 & 1.28 & 379.9 \\
\hline
\end{tabular}


Although the accuracy of the predicted material properties decreases with increasing frequency (this was to be expected given the constant gain factor that was in use), the error incurred may be acceptable from a practical standpoint since the material properties of layers deeper within a specimen often have less of an impact on thermal phenomena occurring at the surface, which is often the case when modeling the thermal IR signature of an armored vehicle. 


\section{Chapter 7}

\section{Method Validation}

A validation of the measured material properties was performed to assess the practical significance of any measurement error in terms of the device's intended purpose, i.e., military vehicle thermal (infrared) signature modeling. To accomplish this, a thermal model of a Patria armored vehicle was developed with armor plates corresponding to the measured thermal properties of a $1 / 4$ " steel and 3/4" HDPE (high-density polyethylene) stackup (Figure 7.1).

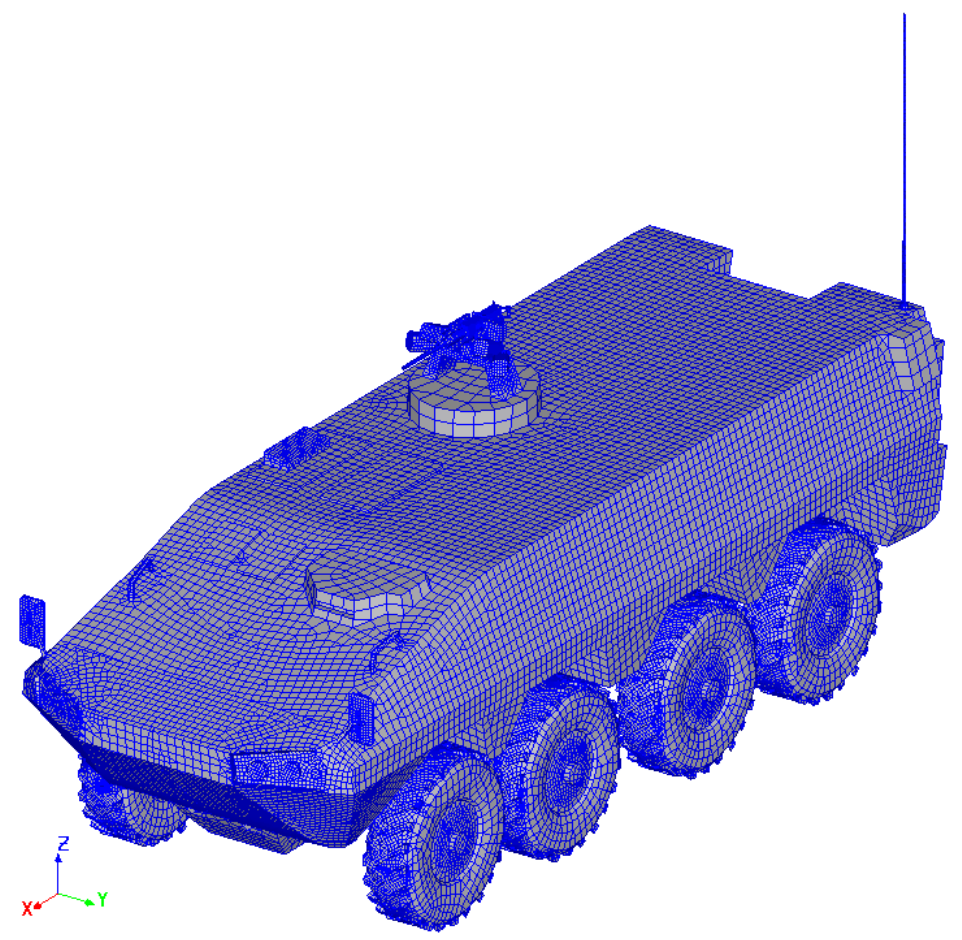

Figure 7.1 - Patria armored vehicle finite-element mesh 
Chapter 7 - Method Validation

The thermal model was exercised in a simulated 24 hour hot desert environment. Hourly thermal and radiance results were compared to those obtained from running a corresponding model constructed using nominal material properties of a steel/HDPE stackup of the same thicknesses. Two additional permutations of the model were created to demonstrate the sensitivity of the vehicle's thermal signature to material property values. Specifically, these models consisted of one with armor represented as 1" steel, and the other as 1" HDPE.

Military vehicle thermal and infrared (IR) modelers evaluate the contrast of the (emitted and reflected) radiance between the vehicle and the surrounding terrain, since the likelihood of detection by enemy forces is dominated by this contrast. Calculating vehicle surface temperatures is the first step in such IR signature analyses; calculating reflected radiance is the second step. To illustrate this, snapshots of predicted thermal and radiance results at an instance shortly after sunrise are shown in Figure 7.2. Note that the radiance prediction includes cold sky reflections off the top of the vehicle. Predicted radiance images for all four vehicle models, i.e., sets of material properties, are also provided in Figure 7.3 for comparison purposes. 
Chapter 7 - Method Validation
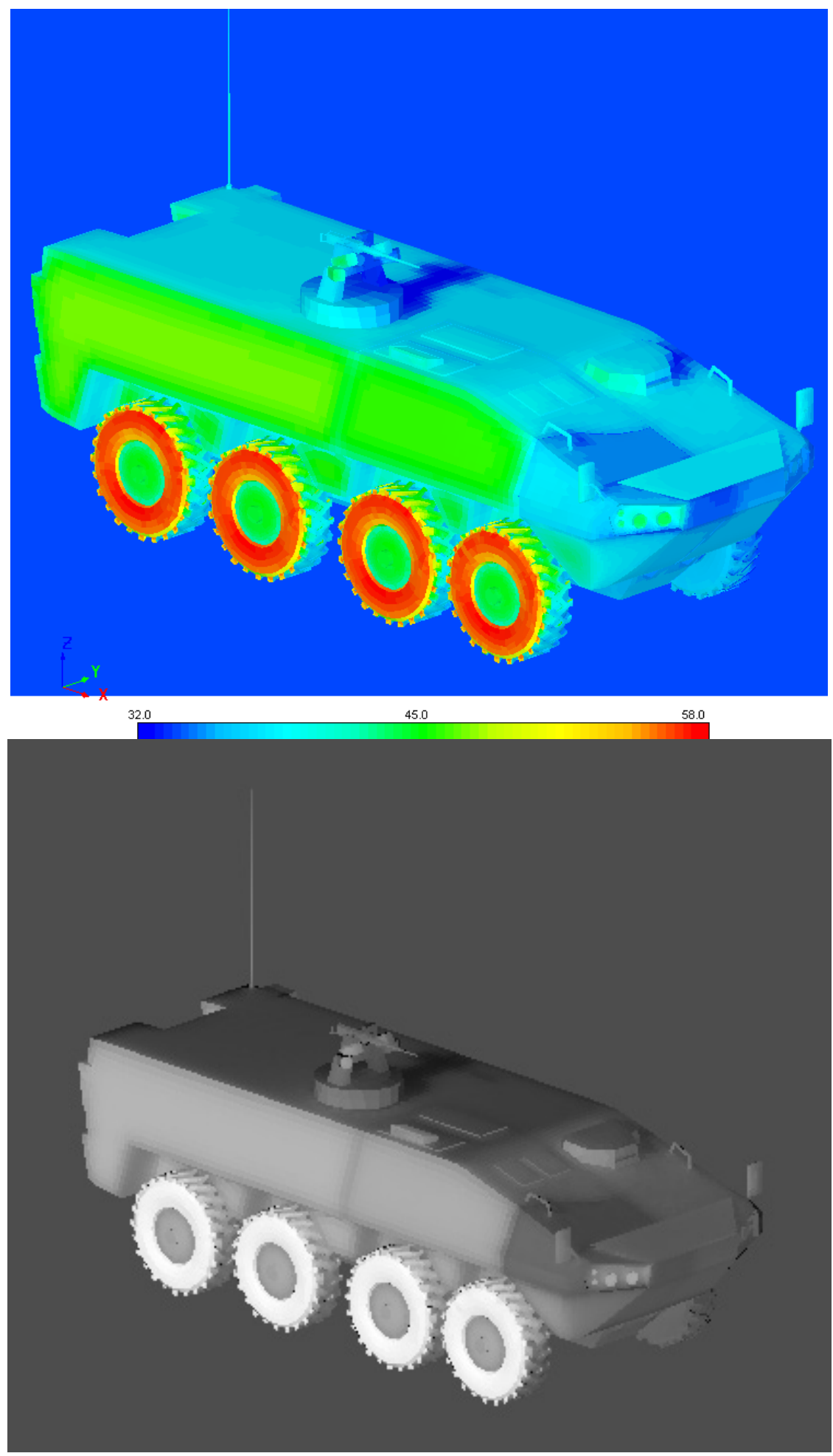

Figure 7.2 - Thermal (above) and radiance (below) predictions of a Patria military vehicle using measured thermal properties of a steel/HDPE stackup as armor 


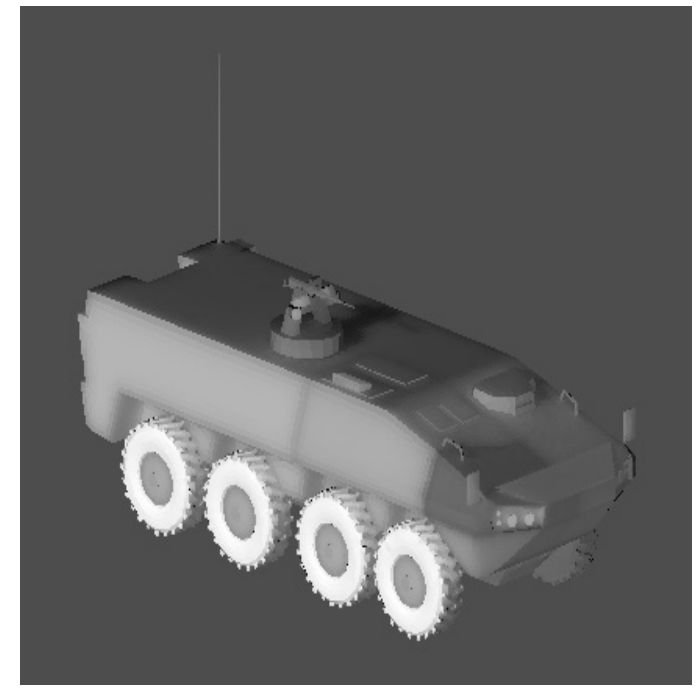

Measured 1/4" Steel over 3/4" HDPE

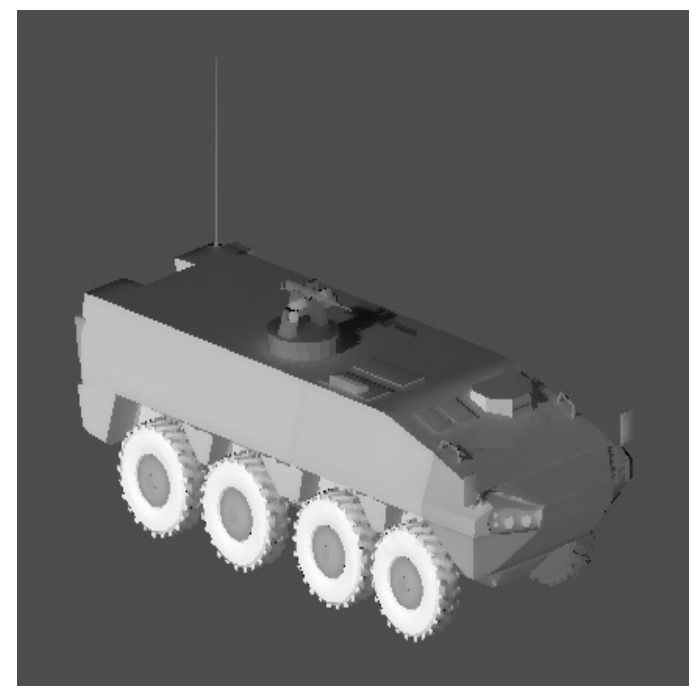

1" HDPE

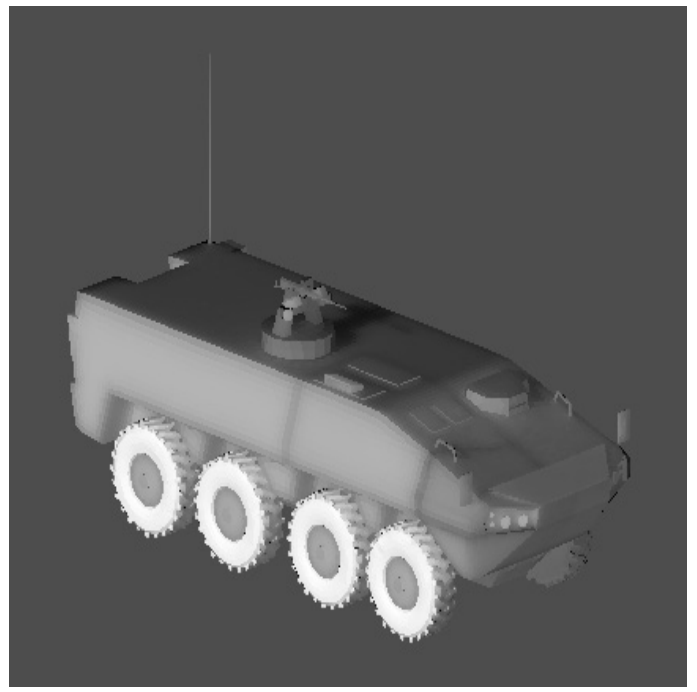

Nominal 1/4" Steel over 3/4" HDPE

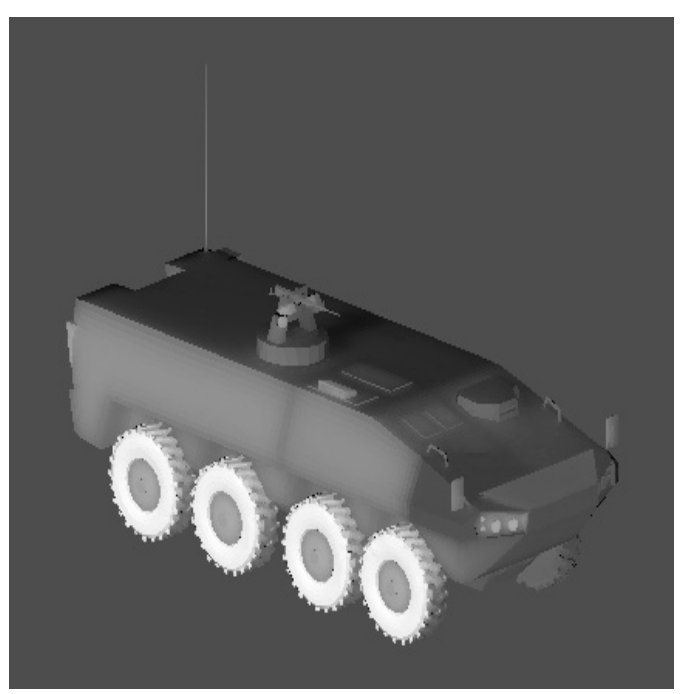

1 "Steel

Figure 7.3 - Comparison of predicted radiances using different armor materials 
A common benchmark criterion of detection for evaluating the IR signature of military ground vehicles is the $\Delta T_{R S S}$ contrast metric.

$$
\Delta T_{R S S}=\sqrt{\left(\overline{T_{T}}-\overline{T_{B}}\right)^{2}+\sigma_{T}^{2}}
$$

The $\Delta T_{R S S}$ contrast metric compares the average "apparent," or equivalent blackbody, temperature of the target, $\overline{T_{T}}$, with the average apparent temperature of the background, $\overline{T_{B}}$, while taking into consideration the standard deviation of the temperatures on the target, $\sigma_{T}$. Evaluation of the $\Delta T_{R S S}$ contrast was performed for each of the four model permutations for the entire 24 hour hot desert scenerio (Figure 7.4).

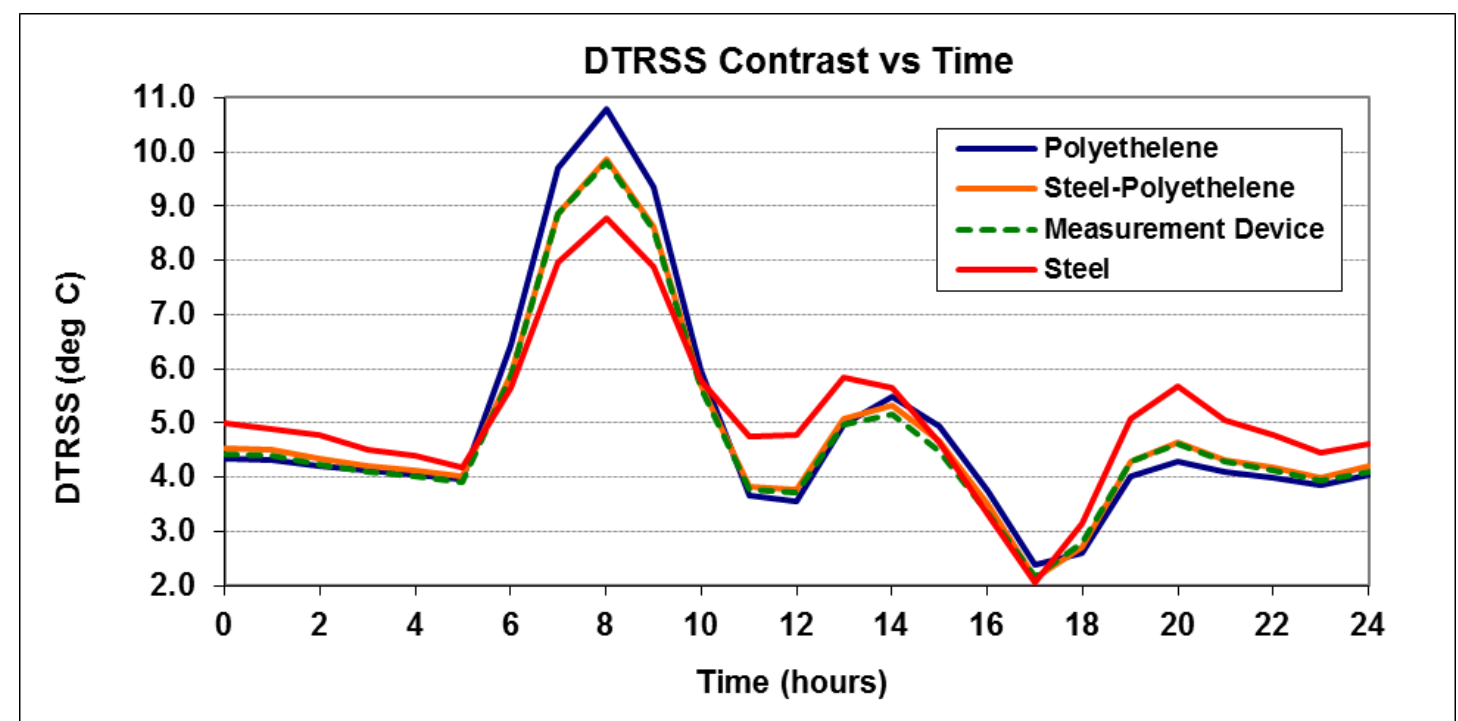

Figure 7.4 - DTRSS contrast results comparison among permutations of the model with various armor properties 
Chapter 7 - Method Validation

The material properties of vehicle armor can have a significant effect on contrast predictions. For example, these results show that after the sun rises at $5 \mathrm{am}$, the vehicle contrasts of the layered and homogenous armor recipes begin to substantially diverge. At 8 am, a $\Delta T_{R S S}$ deviation of approximately $1{ }^{\circ} \mathrm{C}$, which can be significant, is exhibited between the homogenous armor property models and the steel/HDPE stackups. However, a negligible difference in contrast between the simulated (nominal) and measured steel/HDPE is exhibited at this same moment. The contrast of the vehicle incorporating the measured material properties was shown to consistently agree with that of the nominal material properties for the duration of the 24 hour simulation. These results demonstrate the effectiveness of the measurement device for obtaining material properties suitable for this type of analysis. 


\section{Chapter 8}

\section{Conclusion and Recommendations for Future Work}

\subsection{Conclusion}

It has been shown through extensive simulation, testing, and analysis that the material properties of a test specimen can be obtained through a combination of measurement and thermal simulation coupled with an optimization routine. In addition, the layers positioned deeper within a multilayered sample were found to affect the gain and phase of the temperature response of a surface being thermally excited with a sinusoidal heat input. A thermal property measurement device was developed so that physical temperatures and heat fluxes could be obtained from application of a periodic heat source to verify the underlying methodology. Careful analysis of this surface temperature response in conjunction with the heat flux was shown to produce an effective layered representation of a material stack-up, sufficient for characterizing armor for thermal modeling purposes.

\subsection{Recommendations for Future Work}

It was found that the measured heat flux was inaccurate when a temperature gradient across the face of the heat flux sensor was present. A first step in improving the device 
Chapter 8- Conclusion and Recommendations for Future Work should be to ensure that a uniform surface temperature is maintained during transient operation of the Peltier module. Evaluation of a variety of Peltier modules should be performed to assess the magnitude of the temperature gradients developed at the face of the heat flux sensor. Once the optimal Peltier module is found, further evaluation should be performed to obtain an appropriate heat spreader (if necessary), such as a thin copper or TPG (thermal pyrolytic graphite) plate, to be placed between the face of the Peltier module and the heat flux sensor.

Reduction of the temperature gradient across the heat flux sensor should substantially improve correlation between measurements and model predictions. This could be demonstrated by obtaining the gain and phase of the frequency responses for various materials/stack-ups, similar to what was accomplished during this project. Property determination could subsequently be performed which should result in consistent material properties obtained across all frequencies without the need for application of a heat flux gain factor.

Although satisfactory results were obtained to prove the fundamental elements of the property determination approach for homogenous material specimens, improvements to the approach could be made for finding the thermal properties of thermally conductive materials. Prohibitively large models comprised of finely discretized meshes are required to replicate the measured thermal transients captured by the device when operating at high frequencies. To address this issue, development of a device comprised of a circular 
Chapter 8- Conclusion and Recommendations for Future Work heater and heat flux sensor may allow for 2-D models to be employed that make use of inherent symmetry to reduce the computation time required to derive thermal properties.

Additionally, in order to improve measurement of underlying layers, a frequency domain analysis of the measured thermal signals could be performed to determine material properties, or at the least, establish realistic bounds for the properties being solved for. This could be coupled with a combination of ultrasound and thermal approaches that could complement one another in improving the accuracy and precision associated with material property determination. 


\section{References}

[1] ASTM Standard E1225, 2009, "Standard Test Method for Thermal Conductivity of Solids by Means of the Guarded-Comparative-Longitudinal Heat Flow Technique," ASTM International, West Conshohocken, PA, 2009.

[2] Hooper F.C., Lepper F.R., "Transient heat flow apparatus for the determination of thermal conductivities," ASHVE Transactions, 1950, 56, 309-324.

[3] Eustachio D., Schreiner R. E., Transactions of the American Society of Heating and Ventilation Engineers, Volume 58, 1952, p. 331.

[4] IEEE Standard 442-1981 "Guide for Soil Thermal Resistivity measurements," ISBN 0-7381-0794-8.

[5] ASTM Standard D5334, 2008, "Standard Test Method for Determination of Thermal conductivity of Soil and Soft Rock by Thermal Needle Probe Procedure," ASTM International, West Conshohocken, PA, 2008.

[6] ASTM Standard D5930, 2009, "Standard Test Method for Thermal Conductivity of Plastics by Means of a Transient Line-Source Technique,” ASTM International, West Conshohocken, PA, 2009.

[7] ISO 22007-2 "Plastics - Determination of thermal conductivity and thermal diffusivity - Part 2: Transient plane heat source (hot disc method)"

[8] ISO 22007-3 "Plastics - Determination of thermal conductivity and thermal diffusivity - Part 3: Temperature wave analysis method" 
[9] ISO 22007-4 "Plastics - Determination of thermal conductivity and thermal diffusivity - Part 4: Laser flash method"

[10] Krishnaiah S., Singh D.N., Jadhav G.N. "A methodology for determining the thermal properties of rocks,” Int. J. Rock Mech. Mining 2004, 5, 877-882.

[11] ASTM Standard E1530, 2011, "Standard Test Method for Evaluating the Resistance to Thermal Transmission of Materials by the Guarded Heat Flow Meter Technique,” ASTM International, West Conshohocken, PA, 2011.

[12] ASTM Standard C518, 2010, "Standard Test method for Steady-State Thermal Transmission Properties by Means of a Heat Flow Meter Apparatus,” ASTM International, West Conshohocken, PA, 2010.

[13] ISO 8302 "Thermal insulation - Determination of steady-state thermal resistance and related properties - Guarded hot plate apparatus"

[14] ASTM Standard C177, 2004, "Standard test method for Steady-State heat Flux Measurements and Thermal Transmission Properties by Means of the Guarded-HotPlate Apparatus," ASTM International, West Conshohocken, PA, 2004.

[15] Liu, J., 1984. "Temperature Dependence of Elastic Stiffness in Aluminum Alloys Measured with Non-contact electromagnetic Acoustic Transducers (EMATS)”. 1984 Ultrasonics Symposium, pp. 972-974. Ed. B. R. McAvoy, IEEE, New York.

[16] Myers, M., Walker, D., Yuhas, D., Mutton, M., 2008. "Heat Flux Determination From Ultrasonic Pulse Measurements”. Proceedings of IMECE, IMECE200869054. 
[17] Incropera, F., Dewit, D., 2002. Fundamentals of Heat and Mass Transfer, Fifth Edition. New York: John Wiley and Sons. 


\section{Appendix A}

\section{Sinusoidal Inputs and Response: Starting Phase Considerations}

Application of a sinusoidal heat flux to the surface of a homogeneous sheet of material (with nominal capacitance and an adiabatic backside) will typically result in a front side temperature response that exhibits both a phase shift as well as a shift along the ordinate axis relative to its starting temperature. The amount of shift is a function of the magnitude and starting phase of the forcing function relative to the pure cosine function and the heat capacity and thermal conductivity of the material. When performing a thermal analysis using a multi-node thermal model, it can be seen that the magnitude of the shift is also dependent on the fidelity of discretization through the thickness. The direction of the shift is dependent on the starting phase of the forcing function.

For example, when a pure sine wave forcing function is applied to a multinode numerical model of a thick steel slab whose initial temperature is zero, the following transient surface temperature response can be obtained. 
Appendix A - Sinusoidal Inputs and Response: Starting Phase Considerations

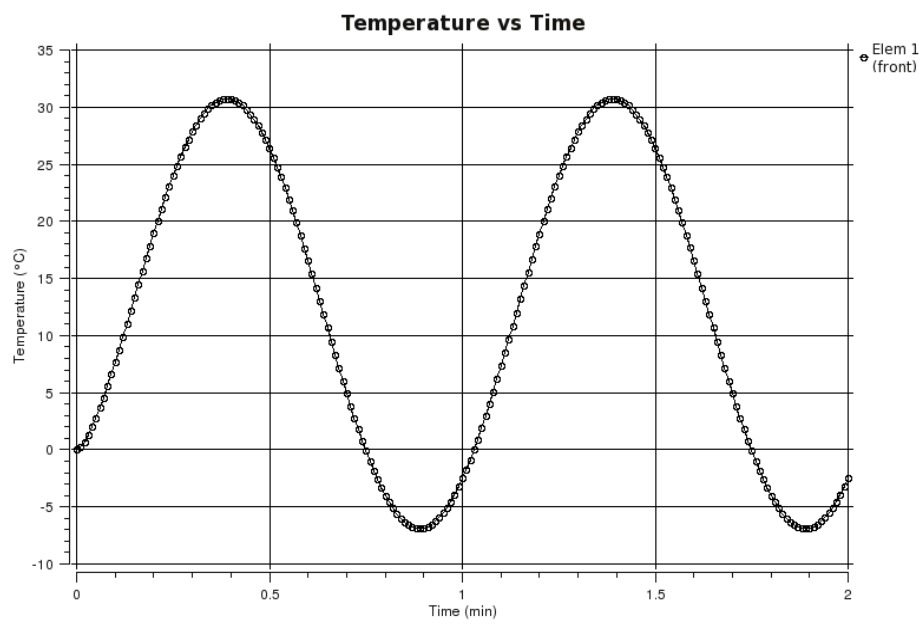

Figure A.1 - Positive sine input produces response that is shifted up along ordinate axis

By shifting the input signal by $180^{\circ}$ (i.e. applying a negative sine wave) an almost identical result can be obtained, with the only difference being that the signal is reflected about the time axis at the location of the initial temperature.

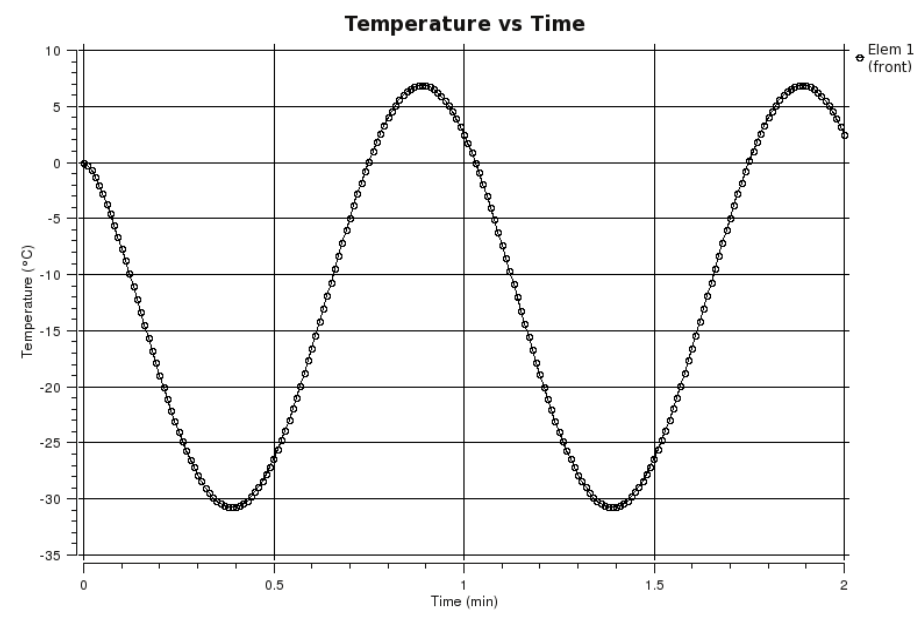

Figure A.2 - Negative sine input produces response that is shifted down along ordinate axis 
However, for the special case of a pure cosine forcing function $\left(0^{\circ}\right.$ starting phase shift $)$, the response of the front side surface temperature shows a tendency to oscillate about its starting temperature, regardless of the amount of heat capacity present.

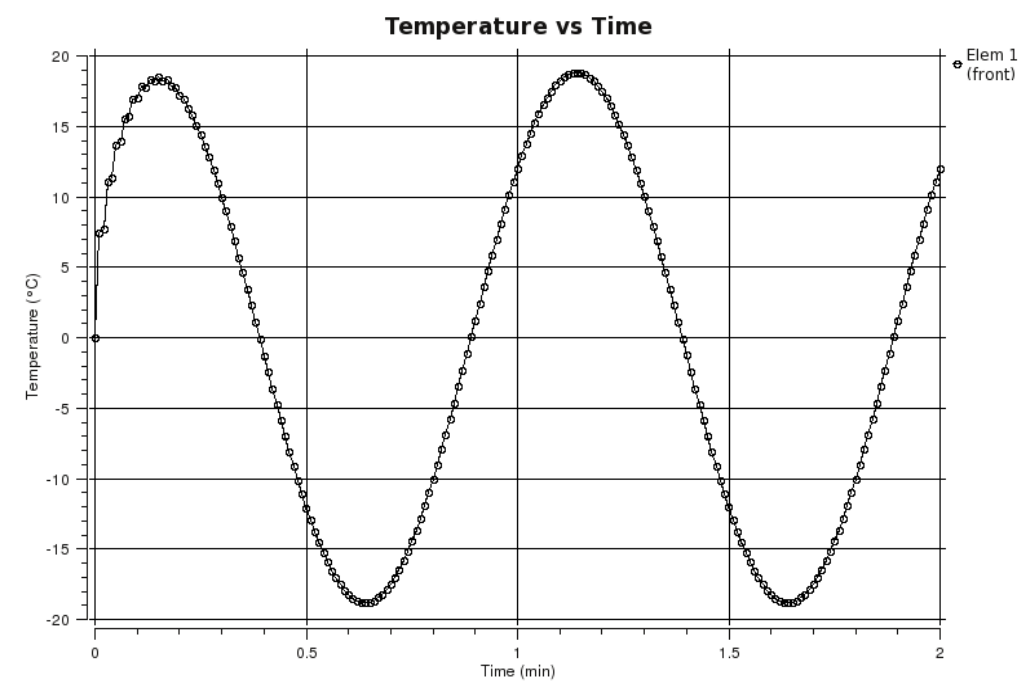

Figure A.3 - Cosine input produces response that oscillates about its starting temperature

As the heat capacity is decreased (or if the fidelity of discritization is diminished, as in a lumped capacitance model), it can be shown that an input + sine based forcing function produces a temperature response whose minimum value is equal to its starting value. 
Appendix A - Sinusoidal Inputs and Response: Starting Phase Considerations

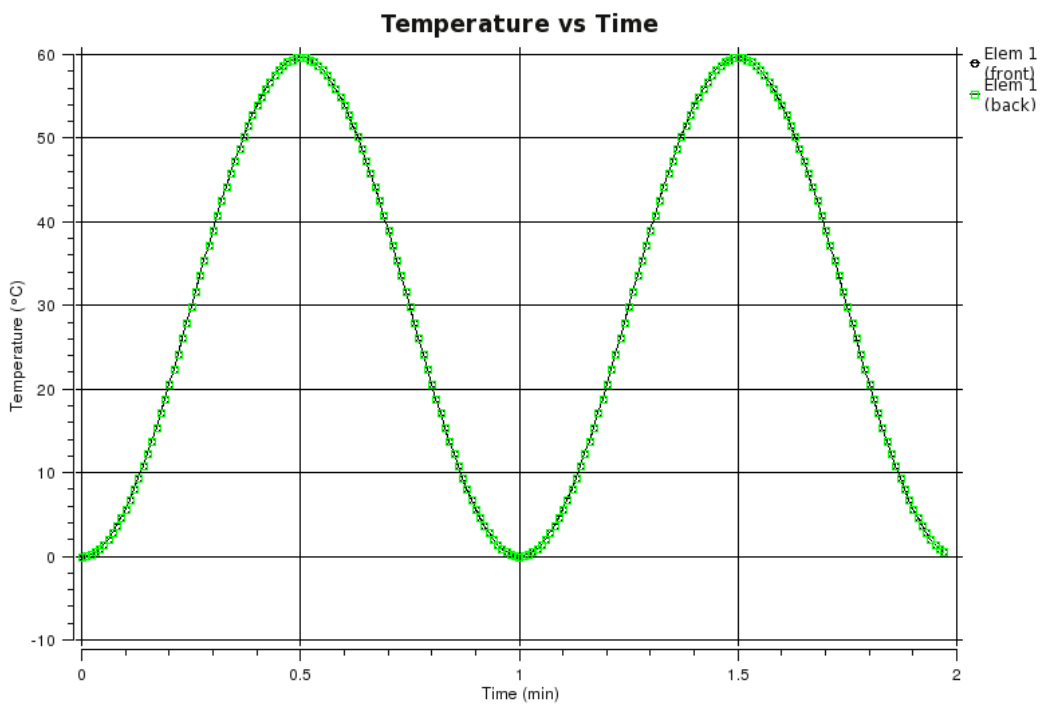

Figure A.4 - Response for small capacitance or lumped capacitance system given a sine input

The pure cosine input function produces the following front side temperature response, showing that the material properties or the fidelity of discritization in the model does not produce a shift along the ordinate axis; it simply oscillates about its initial temperature. 
Appendix A - Sinusoidal Inputs and Response: Starting Phase Considerations

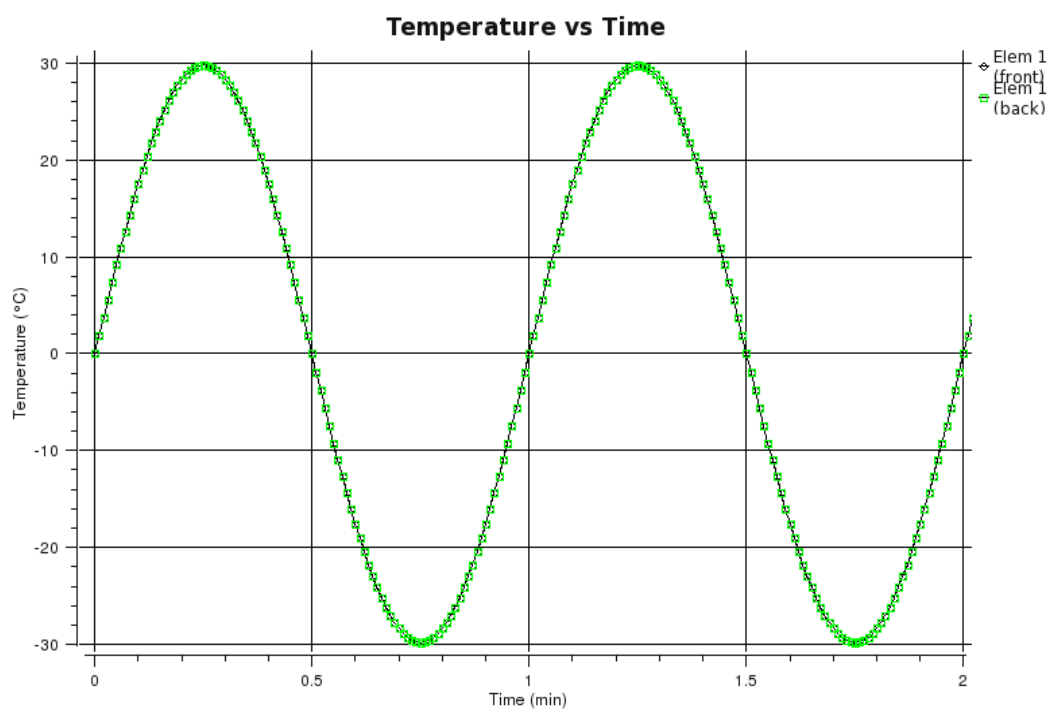

Figure A.5 - Response for small capacitance or lumped capacitance system given a cosine input

When accounting for the accumulated heat received by the front surface, the following results can be obtained for a variety of pure sine and cosine waves. These plots show that, for the $+/$ - sine inputs: 1 ) the net heat input into the system is zero only once during the entire period (at the beginning/end of the input cycle); 2) an entire heating (or cooling) cycle must be performed before the cooling (or heating) cycle can begin; and, 3) that the graph of the accumulated heat received by the front surface is similar in phase to the temperature response of the idealized capacitanceless case. 
Appendix A - Sinusoidal Inputs and Response: Starting Phase Considerations

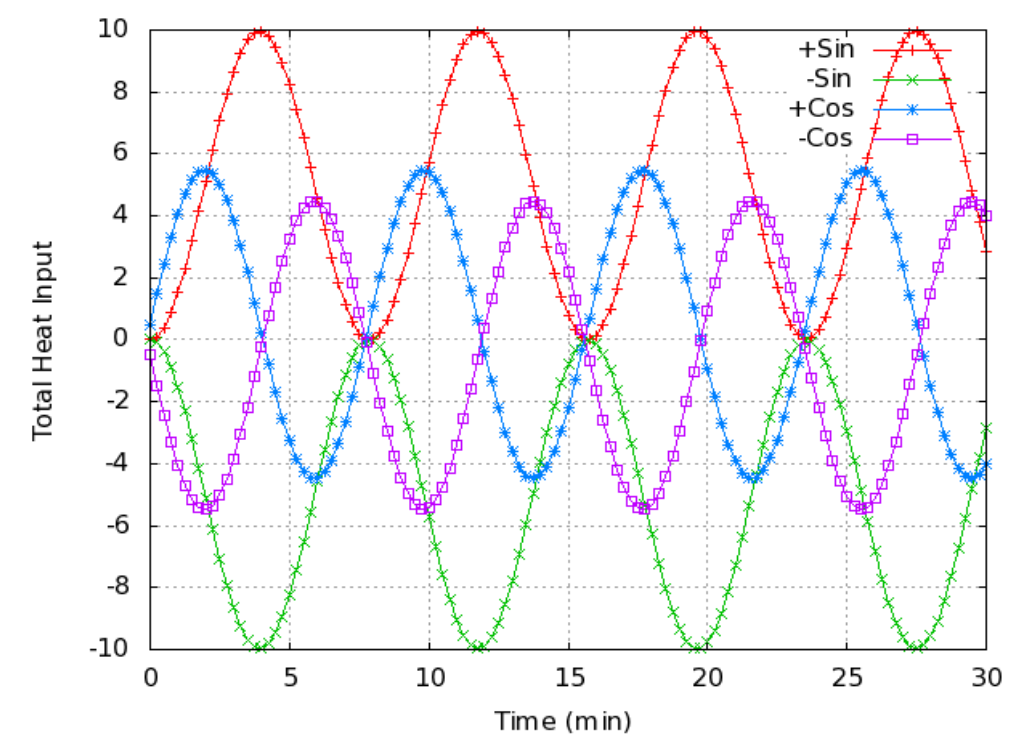

Figure A.6 - Accumulated heat input for different sinusoidal functions

The reason for this shift along the ordinate axis can be explained by a simple theoretical analysis of a lumped parameter system involving only power and energy, given that the temperature in this case is proportional to the net energy into and out of the system (e.g. $T \propto E)$.

Given that the only energy in and out of the system is from the power source, the energy of the system is related to the incident power by a simple time derivative,

$$
P_{i n}=\frac{d E_{s y s}}{d t}
$$

Integrating the incident power with respect to time, we can solve for the energy state of 
the system at any time $t$,

$$
\begin{aligned}
& E_{s y s}=\int_{0}^{t} q d t \\
& \int_{0}^{t} A \sin (\omega t) d t=[-A \cos (\omega t)+A \cos (0)] \\
& E_{s y s}=-A \cos (\omega t)+A
\end{aligned}
$$

The above result shows that the energy accumulated in the system when given a pure sine forcing function follows a negative cosine function shifted along the ordinate axis by an amount relative to the amplitude of the forcing function.

Similarly, integration of the input power consisting of a cosine function provides a special case result in which a sine of zero appears, allowing the energy response to oscillate about its initial state without any shift along the ordinate axis.

$$
\begin{aligned}
& \int_{0}^{t} A \cos (\omega t) d t=[A \sin (\omega t)+A \sin (0)] \\
& E_{s y s}=A \sin (\omega t)
\end{aligned}
$$

Finally, a multinode thermal analysis of the temperature distribution through the thickness, starting from its initial excitation, i.e. $t_{0}=0, t_{1}\left(A=A_{\max }\right), t_{2}\left(A=A_{0}\right), t_{3}\left(A=A_{\min }\right)$, illustrates the transient nature of the problem. The plots in Figure 7 show that a lumped capacitance solution is not valid when performing a quasi-steady state analysis of a 
Appendix A - Sinusoidal Inputs and Response: Starting Phase Considerations material's response to a sinusoidal forcing function. Additionally, a lumped parameteronly analysis may lead one to the wrong conclusion that the surface temperature is indicative of the thermal state of the system as a whole because the model incorrectly represents the entire system and cannot account for the spatial temperature variation within it.

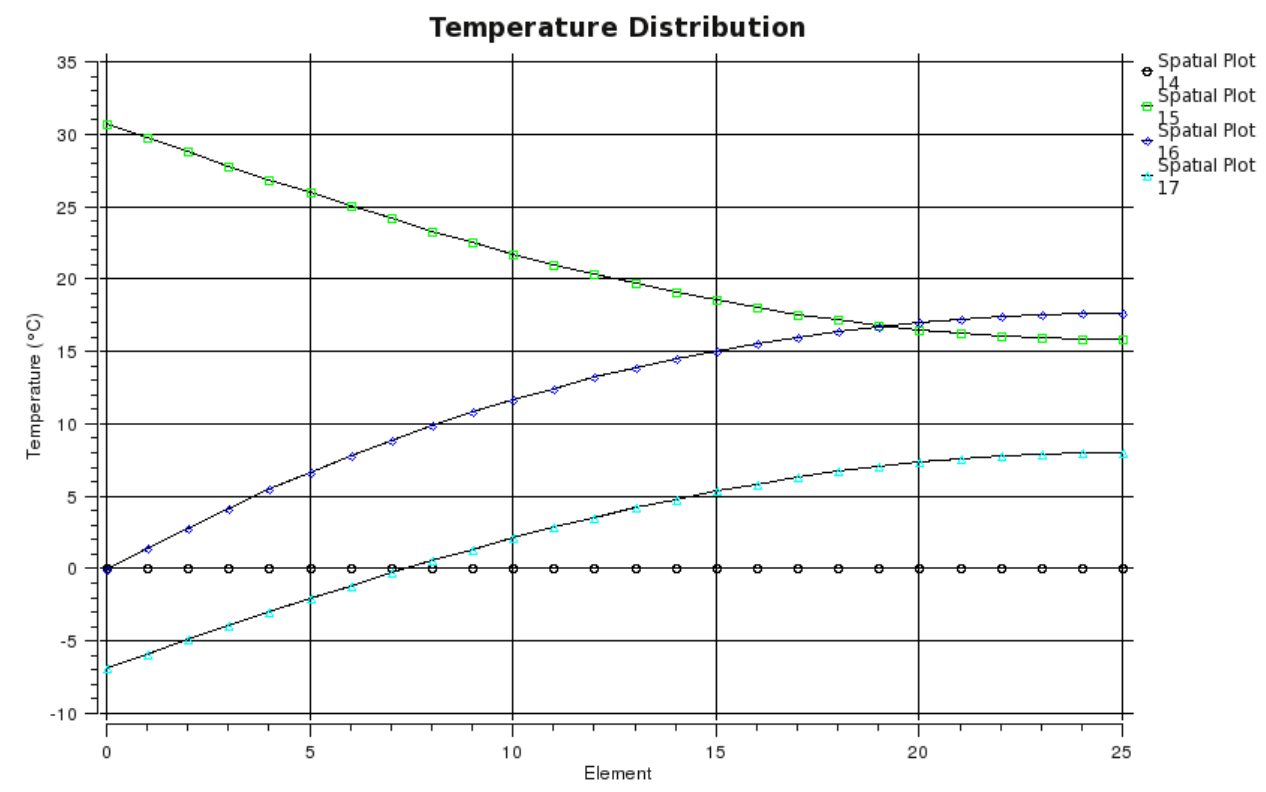

Figure A.7 - Transient temperature profiles at $\mathrm{T} 0, \mathrm{~T}=\mathrm{T} \max , \mathrm{T}=\mathbf{0}, \mathrm{T}=\mathrm{T} \min$

In order to fully understand the front side temperature response, it is necessary to examine the net heat in and out of the front side by performing an energy balance for a theoretical node at that location. This energy balance can be expressed as

$$
\Delta x \rho c_{p, 1} \frac{\left(T_{1}-T_{1}^{\prime}\right)}{\Delta t}=q^{\prime \prime}+\frac{k_{1}}{2 \Delta x}\left(T_{2}-T_{1}\right)
$$


Appendix A - Sinusoidal Inputs and Response: Starting Phase Considerations

Note that this equation shows that the front side temperature is not just a function of its

heat capacity, conductivity and the input heat, $q^{\prime \prime}$, but also a function of the thermal state of the adjoining subdivisions (layers) deeper within the system. The energy balance for the "next node in" is also a similar function of the material properties and internal temperatures,

$$
\Delta x\left(\rho_{1} c_{p, 1}+\rho_{2} c_{p, 2}\right) \frac{\left(T_{2}-T_{2}^{\prime}\right)}{\Delta t}=\frac{k_{1}}{2 \Delta x}\left(T_{1}-T_{2}\right)+\frac{k_{2}}{2 \Delta x}\left(T_{3}-T_{2}\right)
$$

This analysis demonstrates that a pure sine input heat function can cause the surface temperature to rise to its maximum value and subsequently fall below its starting point, even though the amount of heat extracted is equal to the amount injected. This is because yet while the heat is being diffused into the slab, the active cooling cycle begins at the surface; so from the surface node's perspective (whose heat capacity is smaller than the system as a whole), the heat that was diffused deeper into the slab is "lost". This phenomenon is illustrated in Figure A.7 in that the temperature of the back node is shown to be higher than its starting temperature at the start of the cooling cycle. Finally, it has been shown that the surface temperature response can be a function of all of the material properties within the system, and the contribution due to these properties can be characterized (at least in theory), as long as the entire system can be excited, that is, the temperatures throughout the system can be forced to oscillate relative to one another. 


\section{Appendix B}

\section{Derivation of a Closed Form Ultrasonic-based Internal Temperature Distribution Equation}

The time-of-flight, $G$, of an ultrasonic signal traveling through a material specimen can be related to its internal temperature distribution, $T(x)$, as shown in [17],

$$
G=\frac{2 L}{c_{i}}-\frac{(\alpha+\gamma)}{c_{i}} \int_{0}^{L} T(x)-T_{i} d x
$$

where $L$ is the thickness of the layer (m), and $\alpha, \gamma$, and $\mathrm{c}_{0}$ are thermally dependent material properties: $\alpha$ is the coefficient of linear expansion $\left(\mathrm{K}^{-1}\right), \gamma$ is the Young's Modulus change with temperature $\left(\mathrm{K}^{-1}\right)$, and $\mathrm{c}_{0}$ is the speed of sound at the reference state $(\mathrm{m} / \mathrm{s})$.

Approximating the temperature distribution within the material as a linear function of the surface heat flux, $q^{\prime \prime}$, thermal conductivity, $k$, and surface temperature, $T_{s}$,

$$
T(x)=\frac{q^{\prime \prime}}{k} x+T_{s}
$$

and integrating, results in the following expression:

$$
\frac{2 L-c_{i} G}{\alpha+\gamma}=\frac{q^{\prime \prime} L^{2}}{2 k}+T_{s} L-T_{i} L
$$

which can be rearranged as 
Appendix B - Derivation of Ultrasonic-based Internal Temperature Distribution Equation

$$
q^{\prime \prime}=\frac{2 k}{L}\left[\frac{2 L-c_{i} G}{\alpha+\gamma}-T_{s} L-T_{i} L\right]
$$

The internal temperature distribution can subsequently be related to the ultrasonic timeof-flight by substituting (B.3) into (B.2),

$$
T(x)=\frac{2}{L^{2}}\left[\frac{2 L-c_{i} G}{\alpha+\gamma}-T_{s} L-T_{i} L\right] x+T_{s}
$$

Finally, by assuming a homogeneous internal temperature distribution at the initial state, i.e., $T_{i}=T_{s}=T(L)$,

$$
c_{i}=\frac{2 L}{G_{i}}
$$

it can be shown that the internal temperature distribution of a homogenous layer of material can be obtained from knowledge of its thickness, material properties, measured time-of-flight, and measured surface and initial temperatures:

$$
T(x)=\frac{4}{L}\left[\frac{1-G / G_{i}}{\alpha+\gamma}-\frac{T_{s}-T_{i}}{2}\right] x+T_{s}
$$


Appendix C - Heat Spreader Investigation

\section{Appendix C}

\section{Heat Spreader Investigation}

An attempt at minimizing the temperature gradient across the face of the heat flux sensor was performed to improve correlation between the simulations and the temperature and heat flux measurements. This was accomplished by integrating two thin copper plates $(1 / 8$ " thick) to act as heat spreaders at the surfaces of the Peltier module, so that a uniform temperature could be maintained on either face. The sizes of these copper plates were machined to be consistent with that of the heat flux sensor $(60 \mathrm{~mm} \times 60 \mathrm{~mm})$. It was expected that such a modification would substantially improve the accuracy of the measured heat fluxes while having a negligible effect on the heat transfer from the heater to the test specimen.

To test the performance of the heat spreaders, the previously described sinusoidal and constant power testing was performed once again on the $1 / 2$ " HDPE calibration coupon. The test setup is illustrated in Figure C.1. Thermal pads were inserted at all interfaces (a total of 5) to reduce the contact resistance between the individual components. 


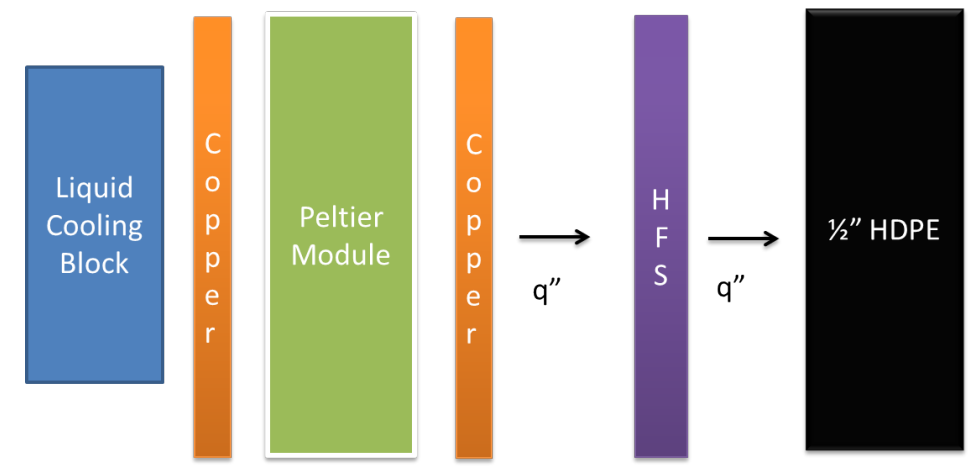

Figure C.1 - Copper heat spreader placement

Integration of the copper heat plates was found to dramatically improve correlation between simulation and measurement, as is evident in Figure C.2 and Figure C.3. Specifically, the gain factor was found to be reduced from its previously determined value of about 2 to a value of approximately 1.3.

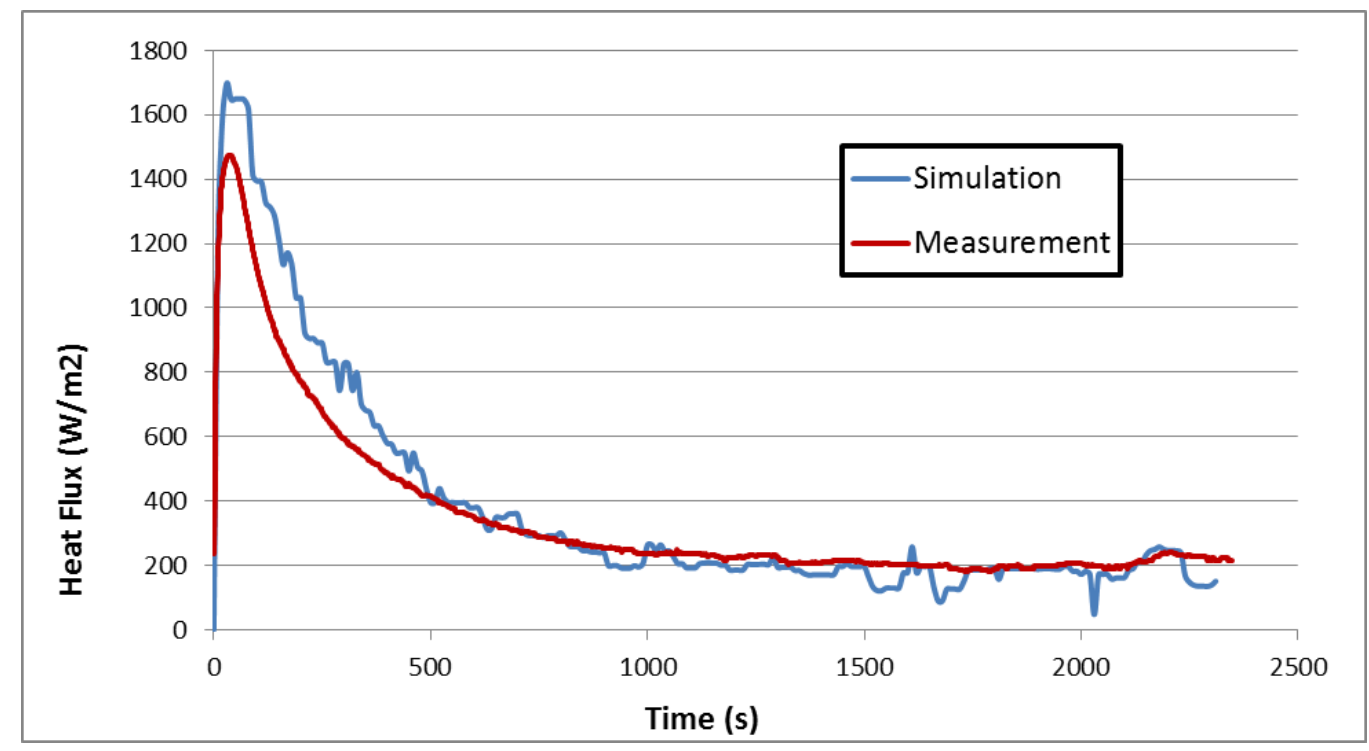

Figure C.2 - Constant power supplied to Peltier module with integrated copper heat spreaders 
Appendix C - Heat Spreader Investigation

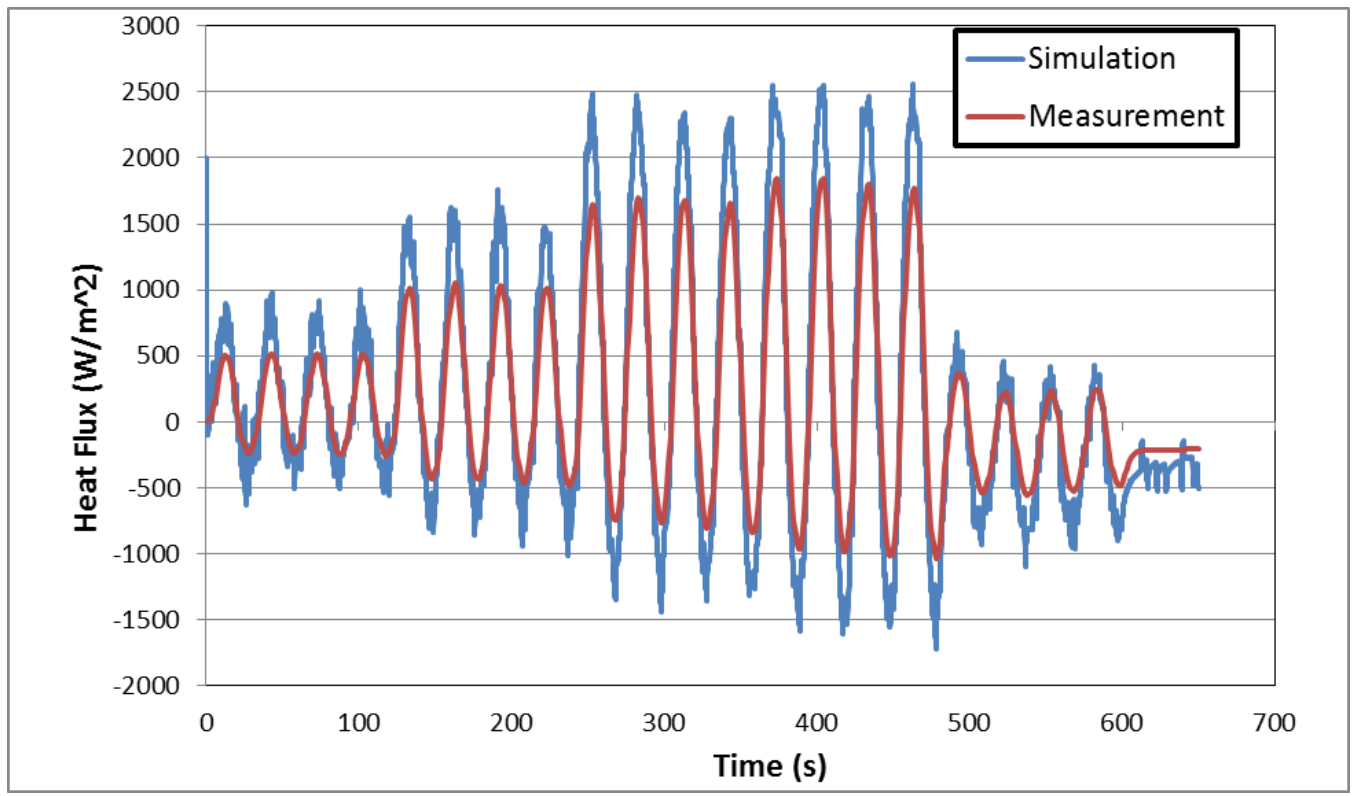

Figure C.3 - Sinusoidal operation of Peltier module with integrated copper heat spreaders

Since modifications to the device to make use of these particular copper heat spreaders still required the use of a non-unity transient heat flux gain factor, it is recommended that further testing be performed using thicker heat spreaders or an improved Peltier module. 